University of Louisville

ThinkIR: The University of Louisville's Institutional Repository

Electronic Theses and Dissertations

$5-2006$

\title{
Individual and situational factors affecting transfer of training in a call center environment.
}

Eric Travis Hicks

University of Louisville

Follow this and additional works at: https://ir.library.louisville.edu/etd

\section{Recommended Citation}

Hicks, Eric Travis, "Individual and situational factors affecting transfer of training in a call center environment." (2006). Electronic Theses and Dissertations. Paper 614.

https://doi.org/10.18297/etd/614

This Master's Thesis is brought to you for free and open access by ThinkIR: The University of Louisville's Institutional Repository. It has been accepted for inclusion in Electronic Theses and Dissertations by an authorized administrator of ThinkIR: The University of Louisville's Institutional Repository. This title appears here courtesy of the author, who has retained all other copyrights. For more information, please contact thinkir@louisville.edu. 


\title{
INDIVIDUAL AND SITUATIONAL FACTORS AFFECTING TRANSFER OF
}

TRAINING IN A CALL CENTER ENVIRONMENT

\author{
By \\ Eric Travis Hicks \\ B.A., Brigham Young University, 1982 \\ M.S., Indiana University, 1984

\begin{abstract}
A Dissertation
Submitted to the Faculty of the

Graduate School of the University of Louisville

in Partial Fulfillment of the Requirements

for the Degree of
\end{abstract}

\author{
Doctor of Philosophy \\ Department of Leadership, Foundations, and Human Resource Education \\ University of Kentucky \\ Louisville, Kentucky
}

May 2006 

Copyright 2006 by Eric T. Hicks

All rights reserved. 
INDIVIDUAL AND SITUATIONAL FACTORS AFFECTING TRANSFER OF

TRAINING IN A CALL CENTER ENVIRONMENT

By

Eric Travis Hicks

B.A., Brigham Young University, 1982

M.S., Indiana University, 1984

A Dissertation Approved on

April 11, 2006

By the following Dissertation Committee:

Dissertation Director 


\section{DEDICATION}

This dissertation is dedicated to my wife, first love, and best friend

Cynthia Dawn Hicks

who has supported and encouraged me with patience, love, and sacrifice during this long journey. Without her at my side we would have never crossed the finish line. 


\section{ACKNOWLEDGEMENTS}

I acknowledge and thank my dissertation committee, Dr. Mike Boyle, who served as Chair, Dr. Joseph Petrosko, Dr. Thomas Reio, Dr. Carolyn Rude-Parkins, and Dr. Thadeus Dumas for their encouragement and support in this long process. In particular, I thank Dr. Boyle for keeping me focused on the most important things and on helping me maintain a positive attitude during the doctoral journey. I thank Dr. Joseph Petrosko for his invaluable assistance and time in helping me find my way through the analytical maze created by this study’s data. I also thank Dr. Reio for his challenge to consider response bias and study limitations. Though not on my committee, I give special thanks to Dr. Paul Winter who challenged me to finish my literature review with appropriate academic rigor, and without whom I would not have been motivated to push for the summit.

No great accomplishment is attained without a great support team and I had the best. My heartfelt thanks and love go to my wife, Cindy, and family for putting up with me during my journey back to school. I also express my love and thanks to my children, Nathan, Kyle, and Whitney, who have helped me keep a perspective on things and given me no shortage of welldeserved teasing about returning to school in my “ancient years.”

I thank my parents who instilled in me a desire to learn and seek out new challenges and in particular my mother, Carol, whose proof reading kept me from making more than a few errors. I also thank my brother, Zac, for providing stimulating discussions during our Saturday morning breakfasts together when I needed an escape and sanity check. Finally, I thank my good friends Dr. Robert Nixon for his enthusiastic encouragement and Marji Settles for her tireless assistance and cheerful smile through all my questions, phone calls and department visits. You're the best! 


\section{ABSTRACT \\ INDIVIDUAL AND SITUATIONAL FACTORS AFFECTING TRANSFER OF TRAINING IN A CALL CENTER ENVIRONMENT}

Eric Travis Hicks

April 11, 2006

In the past 35 years, the average U.S. corporation expenditure per employee on training has increased over ten-fold, yet research studies covering the same time period estimate that only $10-15 \%$ of skills and knowledge acquired during training actually transfer to the job. A study was conducted using Mathieu and Martineau's model of training motivation to better understand which factors predicted for motivation to transfer learning on the job in a call center environment.

Participants from a large mid-western insurance company call center $(n=195)$ participated in a longitudinal study to determine the relationship of individual factors, situational factors, and reaction to training with motivation to transfer training. Pretraining survey scales measured variables motivation to learn, locus of control, selfefficacy, learning confidence, managerial support, organizational support, and job support. Post-training survey scales measured reaction to training and motivation to transfer training.

Pearson correlation, hierarchical multiple regression, and factor analysis were used to understand correlation among the predictor variables and between the predictors 
and the criterion variable motivation to transfer. Results indicated that while all variables of interest correlated with motivation to transfer in Pearson tests, only situational factors and reaction to training predicted for transfer motivation when entered into the final hierarchical multiple regression model. 
TABLE OF CONTENTS

ACKNOWLEDGEMENTS............................................................... iv

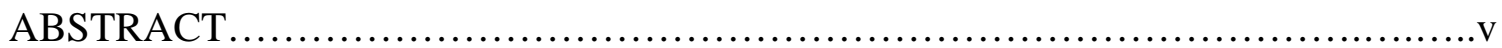

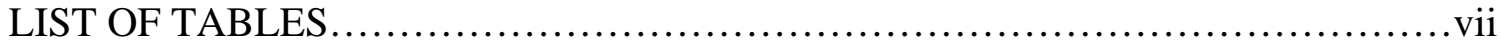

\section{CHAPTER}

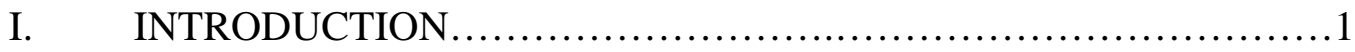

General Background of the Study.....................................

Problem Statement................................................ 5

Professional Significance of the Problem.............................

Theoretical Framework and Methodological Overview................9

Limitations of Previous Studies....................................11

Limitations of Current Study......................................14

II. LITERATURE REVIEW ........................................... 15

Individual Factors Influencing Training Transfer..................16

General Self-Efficacy, Learning Confidence and Locus of Control............................................17

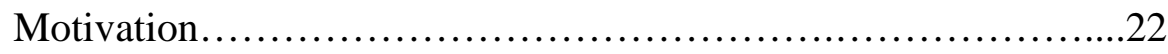

Situational Factors that influence Training Transfer................36

Organizational Climate and Job Support..........................36

Managerial Support..............................................51

Training Design Considerations .................................59 
Practice and Relapse Training....................................63

Transfer Measurement Instruments..............................70

III. RESEARCH METHODOLOGY ......................................72

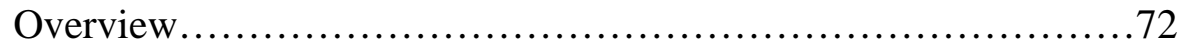

Research Context................................................ 73

Research Questions...........................................74

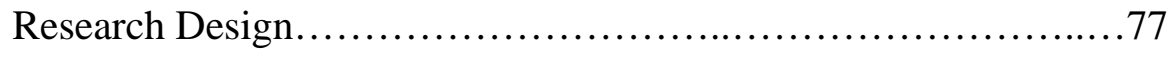

Participants.................................................. 78

Instruments Used in Data Collection................................79

Linking the Surveys.............................................. 80

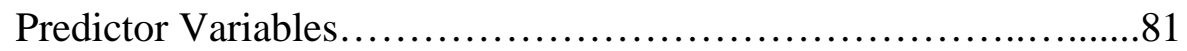

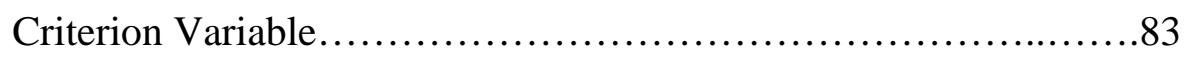

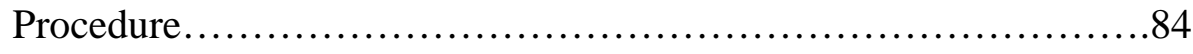

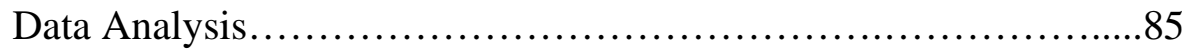

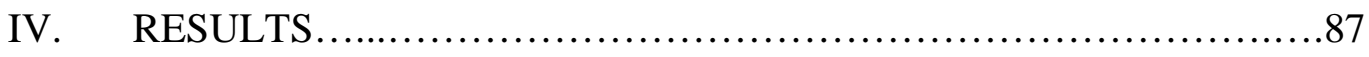

Summary of Characteristics of the Sample...........................89

Summary of Reliability Coefficients for Each Scale..................93

Relationships Among the Predictor Variables........................94

Demographic Correlations........................................96

Individual Variable Correlations....................................96

Situational Variable Correlations.................................97

Reaction to Training Correlations.................................97

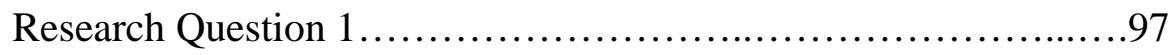


Research Question 2.........................................99

Research Question 3..............................................101

Research Question 4..........................................101

First Regression Analysis....................................102

Second Regression Analysis with Reduced Number of Predictor Variables..................................................106

Summary ....................................................111

V. DISCUSSION, IMPLICATIONS \& RECOMMENDATIONS.........113

Introduction.................................................

Statement of the Problem...............................................114

Review of the Theoretical Model and Method.......................115

Summary of Results..........................................116

Measures used...............................................117

Findings and Implications for Questions 1, 2, and 3................118

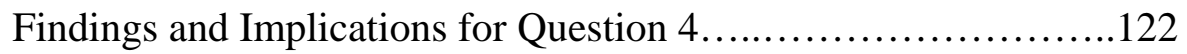

Recommendations................................................124

Study Limitations.............................................126

Suggestions for Additional Research.............................129

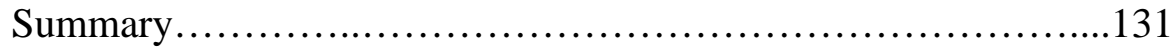

REFERENCES............................................................

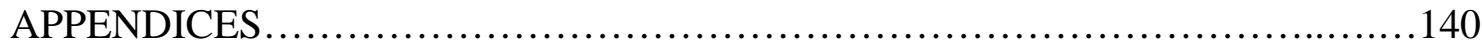

CURRICULUM VITAE .................................................... 150 


\section{LIST OF TABLES}

\section{TABLE}

1. Study Response by Survey.............................................. 89

2. Distribution of Respondents by Gender....................................90

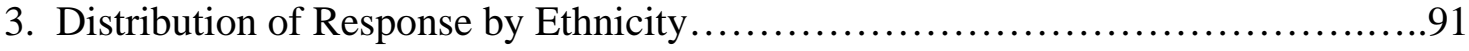

4. Distribution of Respondents by Age.....................................91

5. Distribution of Respondents by Education Level............................82

6. Distribution of Respondents by Years of Experience..........................93

7. Reliability Statistics for Nine Scales................................. 94

8. Means, Standard Deviations and Intercorrelations for Motivation to Transfer and Predictor Variables............................95

9. $R$ Squared Statistics and Standard Error for Four Regression Models..............104

10. Hierarchical Regression Model Summary: Motivation to Transfer Training......105

11. Factor Component Matrix for Individual Characteristic Variables...............107

12. Factor Component Matrix for Situational Characteristic Variables...............108

13. $R$ Squared Statistics and Standard Error of Estimate for Four Regression Models using Individual Factors and Situational Factors.....................109

14. Hierarchical Regression Model Using Situational and Individual Factors Summary: Motivation to Transfer Training..............................110 


\section{CHAPTER 1}

\section{$\underline{\text { INTRODUCTION }}$}

In recent years the workplace has experienced monumental changes in emerging technologies, new competitive markets, globalization, and workplace diversity. Parallel with these changes, organizations have increased the training expenditure to meet the demands of these challenges every year for the past six years (Sugrue \& Rivera, 2005). Unfortunately, the return on the training investment has not increased with the changes. In 1970, the average expenditure on training per employee was approximately $\$ 75$ (Facteau, Dobbins, Russell, Ladd, \& Kudisch, 1995). Today that number ranges from \$1000 to \$1400 (Sugrue \& Rivera, 2005). Every year, U.S. corporations spend billions of dollars on training and development interventions targeted at improving employee performance (Baldwin \& Ford, 1988; Broad and Newstrom, 1992; Noe, 1986,). Yet for the past 20 years researchers have estimated that only $10-15 \%$ of skills and knowledge acquired during training are actually used at the appropriate time on the job (Curry, Caplan, \& Knuppel, 1994; Facteau, Dobbins, Russell, Ladd, \& Kudisch, 1995;

Georgenson, 1982). The gap between training expectations and actual application is not a new problem. In 1957 James Mosel commented that "there is mounting evidence that shows that very often the training makes little or no difference in job behavior (Mosel, 1957).” This transfer “gap” exists in virtually all performance interventions and given 
the large and growing cost of providing training, researchers and business owners alike are concerned about what can be done to increase the return on training investment (Broad, 2005; Broad \& Newstrom, 1992;).

The transfer of training from classroom or PC workstation to the job site involves training related factors such as design, content, and curriculum (Baldwin \& Ford, 1988), trainee-related factors such as motivation and self-efficacy (Noe, 1986), and work environment factors such as supervisory influence and organizational climate (Brinkerhoff and Montesino, 1995; Broad \& Newstrom, 1992). Prior to 1984, however, most training research was based on training design related studies and very few focused on individual or situational training transfer factors. Over the past 20 years, researchers have generated a great deal of research on training transfer which has suggested an equal if not more important factor than design in obtaining training results (Baldwin \& Ford, 1988, Broad, 2005; Broad \& Newstrom, 1992,).

Even with the shift toward training transfer, very few studies in this body of research have focused on call center employees. Most studies have targeted college students or employees and managers from very diverse industries and job functions. A call center workplace is defined as a division or part of a company or organization that services customers by phone and through back-office systems. Call center employees receive in-bound calls from customers or make outbound calls to customers in capacities such as service, sales, claims adjustment, customer disputes, payments, collections, fraud prevention, account adjustments, or inquiry. There also other call-center jobs in which employees may correspond with the customers via email or conduct investigative research where contact with the customer is less frequent. 
Call center employees deserve training transfer scrutiny for two very important reasons. First, call center employment is growing rapidly in other countries, particularly India and the Philippines. Because of this foreign growth, U.S. call center positions have decreased making it imperative that surviving domestic operations be well trained and efficient in their operation. Second, because of the nature of call center operations, employees are paid to be on the phone and therefore are actually performing their job most of the time they are at work. They usually have a very short window between training and opportunity to apply new skills. This creates a very good opportunity to measure their motivation to transfer as well as their skill application.

This dissertation is a report of a correlational study of individual and situational characteristics and their relationship to training transfer motivation within a call-center environment. The study was based on survey responses collected from call-center employees in a large health insurance corporation. The first chapter of the dissertation presents the study background, identifies the problem statement, and outlines the theoretical model and methodology. The chapter concludes by discussing the delimitations of the study and defining special terms used.

\section{General background of the study}

Traditional research in training effectiveness has focused primarily on factors contained within the construct of the training itself. Researchers have conducted studies that well document the relationship between training strategies and training results (Wexley \& Baldwin, 1986; Wexley \& Latham, 1981). 
While the research literature contains many studies directed at training strategies and methodologies, relatively few studies exist about what motivates people to transfer learning. Researchers have argued that trainee characteristics such as motivation and attitudes both before and after training should be studied (Campbell, 1989; Tannenbaum and Yukl, 1992). Others have posited that training is unlikely to have effectiveness unless trainees have a high level of pre-training motivation (Wexley and Latham, 1991). As an antecedent to motivation researchers have also asserted that factors must be considered beyond the immediate training program, such as individual and situation influences (Noe, 1986). Other studies support this thinking by suggesting that employers who foster a motivating work environment will create a competitive advantage in the marketplace (Lawler, 1992; Pfeffer, 1994).

It is important to study both situational and individual factors that influence training transfer motivation for several reasons. Situational factors such as managerial support, job support, and organizational support are factors that an organization's management can manipulate and control. Understanding how these factors affect both pre and post training motivation to transfer skills can help an organization invest more appropriately for a greater return on training investment. Individual factors such as self efficacy, learning confidence, motivation to learn, locus of control, and work intensity are characteristics an employee generally brings to the job as part of his or her psychological make-up. Understanding how these characteristics influence motivation to transfer can help organization leadership refine the selection process to attract employees with attributes. 
Finally, researchers have studied situational and individual factors in many settings, but have conducted very little research within call centers. Call centers present an important focus for training transfer study because of the growing number of organizations using them to provide their primary face to customers. Research in a call center environment is also important given the large number of U.S. jobs that are being shifted off-shore to foreign countries such as India and the Philippines, where the call center industry is growing at an exponential rate (The Economist, 2005). This study will examine the relationship between these factors and motivation to transfer within the call center of a financial services company.

\section{Problem Statement}

The focus of this study was to measure the predictive relationships between situational characteristics, individual characteristics, and reaction to training with the criterion variable post-training motivation to transfer training, in a call center environment. The individual and situational factors used in this study are derived from a training motivation model developed by Mathieu \& Martineau (1997) and further supported by foundational research by Baldwin and Ford (1988) who asserted that similar factors influenced transfer of training.

Mathieu and Martineau classify individual factors as direct summative and efficacy-based measures (Mathieu \& Martineau, 1997). Efficacy-based instruments measure the extent to which participants have a positive self attitude about themselves and their prospective training performance. Gist found that participants who had higher 
self-efficacy before training performed better on training assessments than those who had lower self-efficacy (Gist, Schwoerer, \& Rosen, 1989). Direct summative instruments ask the participant directly about their motivation to learn and transfer training. In this study, the direct summative and efficacy-based measures were self-efficacy, locus of control, motivation to learn, and learning confidence.

Situational factors include organizational, job, and managerial support that either hinder or aid the transfer of learning to the job. Both the pre-training and post- training environment can encourage, discourage, or prohibit the use of newly acquired skills and knowledge on the job (Tannenbaum \& Yukl, 1992). It is important to remember, that unlike situational factors, individual factors are by definition, unique to the personality of the trainee. Situational factors, however, may be shared by one, some, or many participants in a training environment, depending on their shared experiences.

With regard to the criterion variable motivation, many researchers have conducted studies showing that trainees who are more motivated entering the training environment are more likely to perform well in the work environment (Goldstein, 1993; Tannenbaum \& Yukl, 1992). Mathieu asserts that Vroom’s valence-instrumentality-expectancy (VIE) theory produces a measure of training transfer that will best relate to actual work outcomes (Vroom, 1964). Expectancy refers to a person’s personal belief concerning whether he or she can acquire a certain skill. Instrumentality is the perception by that person that the acquisition of the skill will lead to a specific outcome. Valence is the relative desirability of the outcome (Mathieu \& Martineau, 1997).

In this study, efficacy-based, direct summative, and valence-instrumentalityexpectancy measures were used to identify both pre-training and post-training motivation 
to transfer training. Four hypothetical question sets, based on these theoretical constructs, were examined and are summarized as follows:

1. What is the relationship between individual characteristic factors (self efficacy, locus of control, learning confidence, motivation to learn) and motivation to transfer training in a call-center environment?

2. What is the relationship between situational characteristic factors (manager support, job support, and organizational support) and motivation to transfer training in a call-center environment?

3. What is the relationship between post-training reaction and motivation to transfer training in a call center environment?

4. Controlling for demographic variables, what is the predictive relationship between individual and situational variables and motivation to transfer in a call center environment?

A Pearson correlation coefficient was used to determine the nature and magnitude of the relationship between each of the individual and situational factors and motivation to transfer training. After controlling for demographic variables the individual and situational factor variables were loaded into a hierarchical multiple regression analysis to determine the nature and magnitude of the relationship between the three sets of variables. 


\section{Professional Significance of the Problem}

There is a need to improve training transfer within the U.S.-based call center customer service industry. While service positions continue to increase against manufacturing jobs, call center jobs in particular are leaving the U.S. for foreign soil at a rapid pace. One recent study predicts that by 2008 employers will eliminate 130,000 call center service positions as a result of relocating over 3,000 call centers from the U.S. to off-shore locations (Datamonitor, 2004). As a result, companies that wish to continue domestic call center operations must increase efficiency and profitability in order to compete with growing international workforce that will work for lower wages.

Within this context, there is a need to study trainee motivation with respect to training transfer in the call center customer service industry. If call center organizations better understand what motivates employees to transfer the skills and knowledge they learn in training, they can create environments that foster higher transfer rates, and thereby decrease cost and increase productivity. There may also be intrinsic motivators independent from work environment factors that mediate transfer of training on the job. These motivation factors may be ideal hiring profiles for employers to find employees who are better internally motivated to transfer training regardless of the work environment.

The underlying business reason to study transfer motivation is compelling. Within a recent large sample of U.S employers in 2004, the average percentage of payroll invested in learning was $2.52 \%$ and the average expenditure per employee within large organizations was \$1,299 (Sugrue \& Rivera, 2005). The number of hours of formal 
learning per employee is also on the rise from 26 hours per employee in 2003 to 32 hours per employee in 2004 (Sugrue \& Rivera, 2005). While training hours are going up, the relative cost to train employees is also increasing. The average cost per learning hour provided was up to $\$ 642$ from $\$ 595$ for all organizations (Sugrue \& Rivera, 2005). Today, total dollars spent on training exceed $\$ 200$ billion (Holton, Ruona, \& Leimbach, 1998). Despite these large investments, researchers estimate that only $10 \%$ of training expenditures for knowledge and skills results in transfer to the job (Baldwin \& Ford, 1988). Even a marginal increase in transfer efficiency could play a significant role in driving down costs that ordinarily argue for foreign outsourcing.

\section{Theoretical Framework and Methodological Overview}

This study is based on the Mathieu \& Martineau (1997) conceptual model of training motivation (Figure 1). The model asserts that training programs exist in a larger organizational context. While traditional instructional design models focus primarily on the training strategy, Mathieu \& Martineau (1997) contend that both individual and situational characteristics play a large role in determining both learning and on-the-job

training transfer outcomes. Participants come to training with a history of organizational knowledge which they either learned first hand or through peers. This knowledge along with personal characteristics that are hard-wired in the individual learner will influence how they receive and transfer the training back to the job independent of the training design (Mathieu \& Martineau, 1997). It is important to note that while training design 
attributes are not the focus of this research, the line across the top of the model indicates that training program attributes may interact with both individual and situational characteristics and have a direct impact on training effectiveness (See Figure 1).

Training Program Attributes

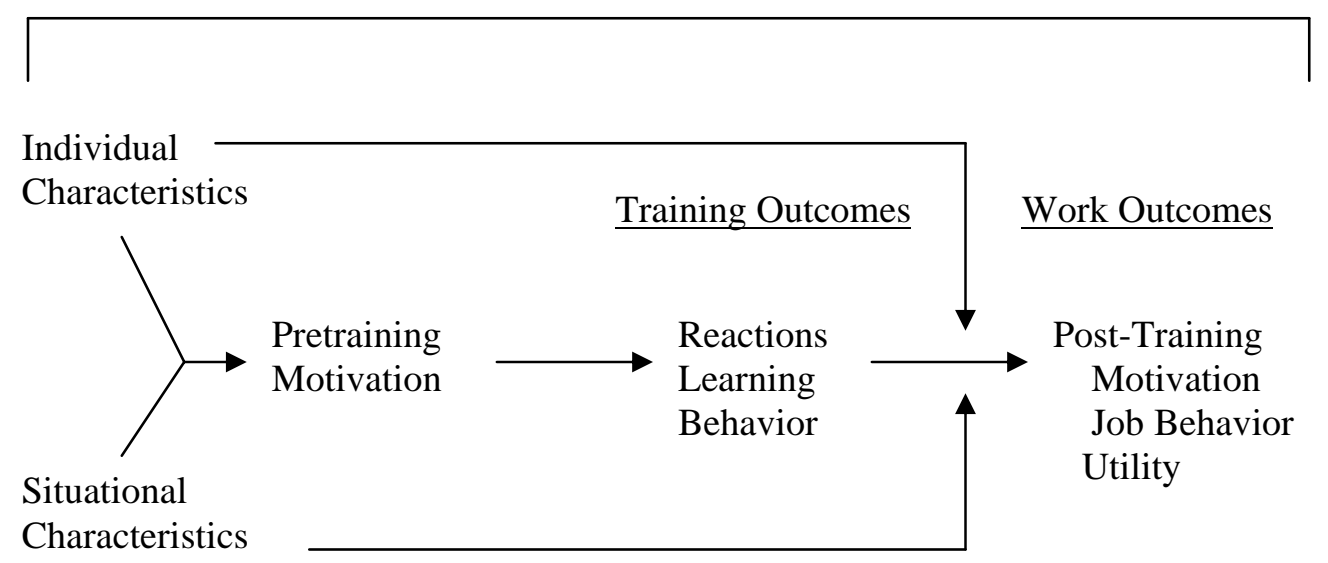

Figure 1: Conceptual Model of Training Motivation (Mathieu \& Martineau, 1997)

The model predicts that learners enter training with different levels of motivation due to differences in situational and personal characteristics. It also predicts that trainees who are motivated to do well in training will learn better than less motivated trainees. More motivated trainees who have learned the concepts and skills will be more likely to apply those concepts and skills on the job (Mathieu \& Martineau, 1997). Other researchers have also established a strong correlation between a trainee’s motivation and learning (Baldwin, 1991; Hicks \& Klimoski, 1987; Martocchio, 1992).

Organizations and call center managers in particular who wish to increase training transfer effectiveness and efficiency should look at both the antecedents to pre-training motivation and the post training factors present in the environment. Several studies support the premise that a trainee's personal characteristics influence his or her 
motivation to learn and transfer skills. Self-efficacy, motivation to learn, locus of control, learning confidence, and work intensity are factors with empirical linkage to training transfer (Brown \& Leigh, 1996; Gist, 1989; Noe, 1986; Warr \& Birdi, 1999). Other Studies indicate that post-training environmental factors such as supervisory support and organizational climate can encourage, discourage, and in some cases prohibit the transfer of new skills and knowledge to the job (Tannenbaum and Yukl, 1992).

The methodology used in this study is a longitudinal correlational design examining the relationship of predictor variables representing situational and individual factors to a single criterion variable, the trainees' motivation to transfer training. Participants were selected from recurrent training classes in a large health insurance company's call center operations. Trainees completed a questionnaire at the beginning of their training and immediately after training. Statistical tests used in this study include Cronbach's alpha for instrument reliability, pairwise correlation analysis to determine inter-correlation among variables, and hierarchical regression analysis on the criterion variable of motivation to transfer. More detailed information on the methodology used is included in Chapter III.

\section{Limitations of Previous Studies}

While several previous studies address the relationship of individual and situational characteristics toward motivation and training effectiveness (Mathieu, Tannenbaum, and Salas, 1992; ETC), there are no known studies which investigate these variables in the context of a call center environment. Additionally, while studies 
generated on training transfer over the past 30 years have been enlightening and directionally helpful for future research, they have been inconsistent in reproducing comparable results between contexts.

Several previous studies have examined the impact of individual and situation characteristics on both training motivation and training effectiveness (Huczynski and Lewis, 1980; Mathieu, Tannenbaum, \& Salas, 1992; Van der Klink, Gielen \& Nauta, 2001; Warr, Allan, \& Birdi, 1999; Warr \& Bunce, 1995). Huczynski and Lewis (1980) conducted research on how organizational factors influenced post-training motivation to transfer. The researchers found that motivation to transfer is more likely to occur when the employee's manager 'sponsored' the training and openly supported attendance. While the study found a link between a situational influence (manager) and motivation to transfer, it did not address personal characteristics such as motivation to learn or self efficacy or how supervisory support might be mediated by the existence of these characteristics. In contrast to these findings, Van der Klink, Gielen \& Nauta (2001) found that groups with supervisors who supported training transfer faired no better than groups with supervisors who did not. Moreover, the researchers found higher transfer was much more significantly linked with trainer influence and trainee characteristics vs. supervisory influence.

Mathieu, Tannenbaum and Salas (1992) investigated a model depicting the relationship between individual and situational influences and both training motivation and effectiveness. Their results supported a link between learning and performance, but only provided minimal support for linking individual and situational characteristics and training motivation. Van der Klink, Gielen, and Nauta (2001) conducted a study 
examining the relationship between supervisor behaviors and transfer of training. Neither study provided compelling evidence that supervisor behavior influenced employee transfer of training. The results of both of these studies are not supported by Broad and Newstrom (1992) nor the Mathieu and Martineau model and therefore merit further investigation of the independent variables.

A survey of the research on factors affecting transfer of training over the past 30 years yields a mixed bag of results. Researchers agree that factors outside of training design have as much if not more influence on training transfer than the design of training itself. What is not agreed upon is the formulary of non-design factors that create transfer improvement. The many experimental and attitudinal training transfer studies recently completed point to significant independent variable to transfer linkage within the context of a specific industry, job design, organization culture, or work environment. The reproducibility of these findings between work environments is scant or tenuous at best. This suggests that previous findings are more context specific than generalizable to all or most work situations.

This research focused on motivation to transfer training within a call-center environment, a context that has been little explored by researchers in the body of the literature. This research also investigated the comparative relationships of key individual and situation factors on motivation to transfer training. While there is prior research on the antecedent factors to motivation, the results vary between contexts and merit further examination. 


\section{Limitations of Current Study}

There primary limitation presented by this study is that common method variance may affect the validity of the conclusions. Method variance is defined as the variance that is attributable to the method of measurement rather than the theoretical construct of

interest (Podsakoff, P. M., MacKenzie, S. B., Lee, J., \& Podsakoff, N. P., 2003). Within this study there are three areas where common method variance may create unwanted covariance between the predictor variables and the criterion variables.

First the criterion variable, motivation to transfer, is by definition a nonproductive outcome that does not represent observable transfer of training to the job. The respondents may say they intend to transfer behavior, but never actually do so. A second potential method variance limitation was the fact that the respondents provided the measurement for both the predictor variable and criterion variable. There is evidence in the research literature that respondents creating measure scores for both predictor and criterion variables may answer in a way inconsistent with their actual behavior (Podsakoff, P. M., MacKenzie, S. B., Lee, J., \& Podsakoff, N. P., 2003). The final limitation caused by method variance may be construct and delivery of the questionnaire itself. The layout and order of questions, particularly in the post-training survey, probably influenced how respondents answered independent from the effect of the variables of interest. These limitations will be address in more detail in Chapter 5 of this study. 


\section{CHAPTER II}

\section{LITERATURE REVIEW}

This study addresses the influence of individual and situation factors on motivation to transfer training. Researchers generally define learning or training transfer as the process that involves the application, generalizability, and maintenance of new knowledge and skills (Ford \& Weissbein, 1997). Both public and private organizations alike invest very large proportions of their total operating budgets on training. Industry analysts estimate that in the United States alone, within companies with more than one hundred employees, $\$ 56$ billion is spent on direct training costs each year. Add to that indirect costs, informal on-the-job training and small company investment, and total training spending could reach well over \$200 billion (Holton III, Ruona, \& Leimbach, 1998).

While the investment in training is large and material to an organization's cost structure, researchers have estimated that only $10 \%$ of the expenditure for new knowledge and skills typically results in transfer to the job (Baldwin \& Ford, 1988). This issue is important to an organization since controlling costs is necessary to growth, reinvestment, and staying competitive in any industry. Business owners want higher rates of return for their investments while eliminating expenditures with low returns.

Mathieu and Martineau (1997) and Baldwin and Ford (1988) identified both individual factors (trainee characteristics) and situational factors (work environment 
characteristics) as two primary components influencing training transfer. These major streams of the training transfer literature will serve as the framework for this literature review. Within the individual factors category, this study will address self-efficacy, motivation to learn, learning confidence, and locus of control. Within the situational factors category we will discuss manager support, organizational support, and job support factors. Within the training design category I will address the following types of studies: goal setting and relapse training. While this training design category is far broader than can be adequately reviewed in this body of work, the focus of this research is on training design only as it includes components that assist participants to transfer learning after the training intervention.

Finally, there have been many improvements in transfer instrumentation within the past few years. Following the major body of the literature review as outlined, we will review Holton's widely accepted Learning Transfer Inventory System and the research that supports it (Holton III \& Bates, 2000).

\section{Individual Factors Influencing Training Transfer}

Individual factors are the trainee characteristics that the participant brings to the training or learning event. They are the lens through which they view both the utility and the context of the training. Two major trainee characteristics which affect transfer include general self-efficacy and motivation. How the trainee perceives his/her own ability to learn and why the learning may be important to the trainee can be enabling or limiting to the overall training objectives. 
General Self-Efficacy, Motivation to Learn, and Locus of control

Self-efficacy, a central component of Bandura’s (1977) social learning theory, is a person's belief in his/her own ability to perform a specific task. There is considerable research in the literature to support the idea that self-efficacy is a key component in transferring skills from the learning environment to the job. While it is an internal attribute that the trainee brings to the learning event, self-efficacy appears to have some effect on external, observable learned behaviors.

Gist, Stevens and Bavetta (1987) conducted a study to examine the effects of self-efficacy on the acquisition of complex interpersonal skills. The participants in the study were MBA students recruited via campus advertisements at a large state university $(N=79)$. The sample was $85 \%$ Caucasian and $62 \%$ male.

The participants attended a program designed to evaluate the effectiveness of negotiating skills training. Post-training negotiation performance was the dependent variable and trainee self-efficacy and post-training goal setting were the independent variables. Participants received four hours of basic negotiation training, after which they completed a written knowledge assessment of the training content and a self-efficacy measure. The researchers measured self-efficacy by asking participants to rate their confidence $(0=$ no confidence, $10=$ high confidence $)$ in achieving various salary levels during the negotiation. The 24-item cognitive learning assessment (alpha $=.72$ ) and the self-efficacy measure (alpha $=.95)$ proved adequately reliable for internal consistency in this study. 
Gist et al. (2001) used Pearson product moment test to find that self-efficacy was associated positively with performance on post-training salary negotiations $(r=.46, p<$ .001). The researchers also found that self-efficacy was significantly correlated with post-training goal setting $(r=.44, p<.001)$. Finally, Gist et al. entered both independent variables self-efficacy and goal setting into a regression analysis and found that the interaction was also significant in predicting negotiation skill $(F(2,73)=4.38, p<.05)$. From these results, the authors contend that high self-efficacy in combination with posttraining goal setting activity can be a significant predictor for training transfer.

Compeau, Higgins, and Huff (1999) conducted a longitudinal study to test the influence of computer self-efficacy, outcome expectations, affect, and anxiety on computer usage. The authors based their research on Compeau's model which states that on-the-job computer usage is based on self-efficacy, expected outcomes, anxiety and personal feelings. Compeau et al. tested 11 hypotheses of which five were directly related to self-efficacy.

The researchers conducted both pretest and pilot studies of the survey instrument and found adequate reliability to proceed with the project. Participants were 2,000 randomly selected subscribers to a Canadian business periodical. The response rate was $53.4 \%$. One year later, the responders received the same survey again. The response rate for the second survey was $67 \%$. The final sample was 394 matched responses from both surveys. For time one, Compeau et al. measured self-efficacy and outcome expectations via a 21-item questionnaire developed by Compeau and Higgins (1995). For time two, Compeau et al. measured affect using the Loyd and Gressard's Computer Attitude Scale (1984). 
The investigators used partial least squares regression analysis adapted to analyze structural equation modeling. Compeau et al. (1999) found that all self-efficacy hypotheses were supported. The results showed that self-efficacy exerted a significant positive influence on performance and personal outcome expectations $\left(R^{2}=9.4 \%, R^{2}=\right.$ 4.6\%), affect $\left(R^{2}=32.2 \%\right)$, and use $\left(R^{2}=28.7 \%\right)$. It exerted a significant negative influence on anxiety $\left(R^{2}=34.3 \%\right)$. The results of this study confirm that self-efficacy has continuing predictive characteristics even one year before behavioral responses are measured. The significance of these findings with regard to training transfer cannot be overlooked.

Cheng (2000) investigated how self-efficacy and personality type affected transfer among MBA students. The participants in the study were students who had already graduated from part-time MBA school in Hong Kong for at least one year but not more than five years $(N=268)$. Seventy-six percent of the participants were male $(n=258)$ while $71.4 \%$ were married $(N=252)$.

To gather data, Cheng (2000) used a multi-item questionnaire which contained 12 validated sub-scales based on prior research, with a response rate of $76 \%$. The researcher then conducted correlation tests among the variables and loaded the data into a structural equation model dictated by the results. Cheng categorized endogenous variables as motivation to learn, perceived skills and knowledge transfer. He categorized the remaining variables as exogenous variables (e.g. self-efficacy, locus of control, personality type). The research design was a structural equation model based on a twostep approach. 
Cheng (2000) found that self-efficacy $(r=.15)$, Type A personality $(r=.42)$, transfer climate $(r=.35)$, and transfer rewards $(r=.30)$ were significant positive predictors of motivation to learn. He also found that the relationship between locus of control and motivation was not significant, supporting previous research by Noe and Schmitt (1986). Both career commitment and job involvement were not related to motivation to learn. This is consistent with previous research on these two factors; (Facteau, Dobbins, Russell, Ladd, \& Kudisch, 1995; Mathieu, Tannenbaum, \& Salas, 1992). Finally, the researcher found that continuous-learning culture and motivation to learn were significant predictors of perceived knowledge $(r=.34, p<.05)$ and skills transfer $(r=.15, p<.05)$. Through these findings, Cheng linked together learner characteristics with motivation, which in turn predicted perceived knowledge and skill transfer.

Tracey (2001) found many factors in addition to instructional content and design influenced not only what people learn, but how they learn it. The researchers conducted a study to determine if pre-training self-efficacy, motivation, and the work environment influenced the relationship between varying levels of training outcomes. The researcher enlisted a private company operating forty hotels located in the southern United States to participate in the study. Approximately 250 managers from these hotels attended a basic managerial knowledge and skills training program. Fifty-one percent of the attendees were female and the trainees averaged 2.3 years tenure with the company and 1.7 years on the job.

Tracey et al. (2001) delivered a packet of four surveys to the trainees one week before every training session, instructing them to complete one and submit the additional 
surveys to their direct supervisor and two co-workers. These surveys assessed the constructs of job involvement, organizational commitment, work environment, pretraining self-efficacy, pre-training motivation, reactions, and learning. The researchers performed a confirmatory factor analysis and determined that the three dimensions of the work environment (managerial support, job support, and organizational support) reflected a single construct, $\chi^{2}(1, N=115)=12.19, p<.01$. An inter-rater agreement index used across all groups $(N=115)$ was 0.74 and indicated a moderately high degree of agreement on the nature of the work environment as a single factor. The research team used all other factors as described.

Through confirmatory factor analysis, Tracey et al. (2001) confirmed the hypothesized model and that the variables job involvement, organizational commitment, and work environment each had significant correlations with pre-training self-efficacy, which in turn had a moderately strong correlation, with pre-training motivation, $r=.51$; $p<.01$. Pre-training motivation correlated significantly, and moderately with four training outcomes: affective reactions, utility reactions, declarative knowledge, and application-based knowledge. Tracey asserted that these findings filled a gap in prior uni-dimensional research which focused solely on knowledge acquisition. By utilizing a multidimensional approach and proving correlation among many factors, Tracey concluded that the transfer process is much more than a single instructional antecedent that results in long term retention and performance.

Self-efficacy, as identified and tested by these researchers plays a significant role in one's ability and willingness to transfer learning, and as such, deserves more study as a principle factor in learning transfer. 


\section{Motivation}

Trainee attitudes and how they affect training outcomes were the subject of much of the transfer research for over 35 years. A large wave of literature argues that when employees perceive that training or some other program has the potential to satisfy their psychological needs, they invest themselves more fully and spend more time and effort attending to the program goals and direction (Kahn, 1990). Motivation can create very powerful learning constructs that often make up for the lack of instructional rigor or positive learning climate. Conversely, well designed training with appropriate follow-up and feedback may not make up for a lack of motivation to learn (Foxon, 1993). While not the only key, motivation is a central component in the puzzle of improving transfer effectiveness.

Ryman and Biersner (1975) conducted an early study investigating the attitudes of new trainees at the Navy School for Divers and their ability to perform their jobs after training $(N=291)$. The trainees' average was 22 years with 11.7 years formal education and 2.6 years of military service. The training included both SCUBA training and deep sea air training. The training content included underwater communication, the use of underwater tools, and diving to 60 foot depths.

There were four major criteria measures for the program: participants who passed the course, participants who asked to quit, participants who were injured or became ill, and participants who failed because of miscellaneous problems. Ryman and Biersner (1975) developed a 25-item questionnaire to measure pre-training attitudes about training motivation, leadership, and course expectations. The researchers conducted a factor 
analysis of the 25 items using principal component analysis. The factor analysis produced two factors from the factor matrix: training confidence and leadership. Factor loadings for both scales were all over .40 .

Ryman and Biersner found that the training confidence factor was significantly related to training success in both of the two major training areas. The leadership scale was significantly related to training success in one of the training areas. The authors asserted that training confidence and leadership were both significant predictors of posttraining performance.

In a similar transfer study, Noe and Schmitt (1986) investigated the relationship between participant's attitudes concerning their careers, jobs, and participation in a training program, and training effectiveness. The researchers hypothesized that motivation to transfer would moderate the relationship between learning and behavior change. In other words, trainees are likely motivated to transfer new skills when they feel confident using the skills, believe that job performance will improve, and believe that knowledge and skills learned in training will help them solve work related problems.

The researchers randomly selected 20 participants each from three principal selection centers $(N=60)$. They selected an equal number of males and females for the study (males $=29$, females $=31$ ) with $66 \%$ of the subjects $(N=40)$ holding nonadministrative positions and the remainder $(N=20)$ holding assistant principal positions (Noe \& Schmitt, 1986). Noe and Schmitt used the School Administrative Descriptive Survey (SADS) to measure attitude of the participants. The SADS survey is an instrument that was designed to measure reactions to assessment of skills, locus of control, job involvement, expectancies, motivation to learn, and exploratory behavior. 
Each of these areas is comprised of scales that exhibit coefficient alpha measurements between .72 and .89 . The researchers used additional surveys and an in-basket exercise to measure training effectiveness in the form of reaction criteria, learning criteria, behavior criteria, and performance criteria.

Noe and Schmitt (1986) asked the training group members to complete the SADS, learning, behavior, and performance measures prior to the training program. They also asked each trainee's supervisor to complete just the learning, behavior, and performance measures. Both participants and supervisors completed the learning, behavior, and performance measures three months after the program's completion. Additionally, participants completed the reaction measure immediately after the training.

The authors performed item analysis, reliability, and construct validity tests on all of the composite scales, finding coefficient alpha scores in the .64 to .95 range, and deemed all scales satisfactory to proceed with the analysis. Noe and Schmitt (1986) used structural path analysis to determine significant causal links between variables. They found statistical significance in the path coefficients between reaction to skill assessment and reaction to training, job involvement and career planning, job involvement and learning, and career planning and behavior $(r=.51, p<.05)$. Trainees who agreed more about their skill strengths and weaknesses as identified in the skill assessment were more likely to believe the content of the training was helpful for skill improvement. Noe and Schmitt also found that job involvement was significantly correlated with learning, behavior, and performance $(r=.45, p<.05)$. Implications of this study suggest that training dollars may be most wisely spent where there is significant agreement by 
participants in their own skill assessment prior to training and where participants are actively involved in their jobs and careers.

Tannenbaum and Mathieu (1991) conducted a study to determine how trainee fulfillment, trainee reactions and training performance would be related to the development of post-training attitudes. The researchers hypothesized that attitudes about the training itself would be positively related to post-training attitudes about the job and motivation to perform. Participants in the study were 1,037 trainees attending recruiting training at the U.S. Naval Recruit Training Command. Of these, 666 (64\%) returned complete data. The average age of the sample was 19.84 years and 368 (55\%) were male.

Within one hour of arriving at the base, Tannenbaum and Mathieu (1991) administered a pre-training questionnaire including demographic data. At the conclusion of the training, the trainees received a questionnaire that assessed post-training motivation, self-efficacy, commitment, training perceptions, and training reactions. The survey instrument contained items rated on a 7-point Likert-type scale $(1=$ Strongly disagree, 7 = Strongly agree). The authors adapted validated scales from past research to assess each of the dependent variables.

Tannenbaum and Mathieu used a three-stage, hierarchical regression with simultaneous entry within stages to test the main hypothesis. The test results provided at least partial support for all three hypotheses. In Hypothesis 1, training fulfillment was positively related to organizational commitment ( $\beta=.201, p<.01$ ), physical self-efficacy $(\beta=.088, p<.01)$, and training motivation $(\beta=.273, p<.01)$. In Hypothesis 2 , trainee reactions were positively related to post-training commitment $(\beta=.318, p<.01)$ and 
motivation $(\beta=.254, p<.01)$ and motivation $(\beta=.254, p<.01)$. In Hypothesis 3 , test performance was positively related to commitment $(\beta=.137, p<.01)$, academic selfefficacy $(\beta=.272, p<.01)$, and training motivation $(\beta=.144, p<.01)$.

These results suggest that pre-training attitudes can be a significant predictor for post training commitment, self-efficacy, and motivation. Practitioners that focus on enhancing pre-training attitudes may better predict post-training outcomes that affect training transferability.

Hicks and Klimoski (1987) conducted a study to determine if the amount of information participants receive about a training program and the amount of freedom they have to do take the program could affect their entry into a training program. Hicks and Klimoski focused their research within a large not-for-profit research and development organization headquartered in Ohio. Managers attending a two-day workshop on performance reviews and interviewing participated in the study $(N=101)$.

The researchers divided the managers into four experimental groups. The first group of managers $(n=33)$ received a realistic training preview and had a low degree of choice in whether they could take the program. Their supervisors sent them a memo telling them they must go to training. The second group $(n=21)$ received a traditional training program announce and had a high degree of choice. The third group received a realistic training preview and no pressure from upper-level managers to take the program $(n=25)$. The fourth group received the low-choice memo from their supervisors and later received a traditional announcement $(n=22)$. Hicks and Klimoski (1987) measured behavior through evaluations of audiotapes of the trainees carrying out simulated performance reviews. Interrater reliability coefficients were acceptable ( $r=.81$ to .89). 
The researchers used a 2 x 2 MANOVA to test for main effects and type of prior information for interaction effects. The MANOVA indicated that participants who received the realistic announcement rated their prior information as significantly more accurate and less positive than those who received the traditional announcement $(F=$ 41.67, $p<.01$ ). Participants with a low degree of choice felt significantly less freedom and more pressure to attend $(F=64.23, p<.01)$ than those who were given a high degree of choice. Hicks and Klimoski (1987) concluded that the information and expectation given to the participants had a significant effect on training outcomes and should be considered when discussing training transfer strategies.

In a similar study, Baldwin and Magjuka (1991) conducted a study to determine the effects of trainee choice of training on subsequent motivation and learning. Students from the University of Indiana representing both traditional full-time students and nontraditional part-time students participated in the study $(N=242)$. The students ranged in age from 20 to 42 and 119, or $49 \%$ were female. The participants signed up for a grouping of management and interpersonal skill training modules provided by an outside training vendor. The researchers divided the participants into three groups and randomly assigned them to three conditions: (a) no choice in modules; (b) choice - but choice not received; and (c) choice - with choice received. Participants in the no-choice grouping received set of modules to complete with no input $(n=84)$. Participants in the choicenot-received grouping evaluated a list of modules from a menu, made a selection, but did not receive their selection $(n=73)$. Participants in the choice-received grouping evaluated a list of modules, made a selection, and then received their selection $(n=50)$. 
Baldwin and Magjuka (1991) used four instruments to measure pre and posttraining motivation, learning, and control for ability. The researchers used the Wonderlic personnel test (Wonderlic, 1985) to assess general cognitive ability. Both the pre- and post-training motivation instruments were the same 7-item scale adapted from previous research by the researcher. The participants completed open-ended measures and shortanswer measures to assess learning outcomes.

Using MANCOVA, the researchers found significant differences between the three conditions with respect to cognitive ability (Pillais $F(6,404)=2.80, p<.01$ ). Univariate tests indicated that the condition of choice had an impact on pre-training motivation and performance on one of the learning measures. As expected pre-training motivation was significantly higher among participants who were allowed choice or whose choice was accepted over those with no choice. Learning was significantly lower on the outcome measures when the chosen training was not received.

This study has implications with regard to training transfer outside of the environmental factors. The knowledge that the training was the participant's choice improved knowledge transfer independent of trainee ability

Facteau and Dobbins (1995) conducted a study to determine whether trainees’ beliefs about the training they would experience actually affected their pre-training motivation and post-training transfer performance. Nine-hundred sixty-seven managers (589 males, 378 females) completed a questionnaire that assessed 14 constructs. The researchers measured the fourteen constructs by using 85 total items on the questionnaire. While many of the survey questions were comprised of different scales that had been 
used in previous research, a 5-point Likert-type scale ( 1 = Strongly Disagree, 5 = Strongly Agree) measured responses for all items.

Facteau and Dobbins (1995) performed a confirmatory factor analysis of the a priori measurement model. Chi-Square test results indicated a goodness of fit (GFI) index of .79 on the revised model which was acceptable for this study. The authors then performed a simultaneous regression of each of the endogenous variables in the model on their predicted antecedents. The resulting structural path model also yielded a .79 GFI. The results of the analysis indicated that of the four social support constructs (subordinate support, peer support, supervisor support, and top management support), only supervisory support was positively correlated to pre-training motivation $(r=.12)$, but it was negatively correlated to perceived post-training transfer $(r=-.09)$. Both subordinate support $(r=.21)$ and peer support $(r=.37)$ correlated positively with perceived posttraining transfer. In general, pre-training motivation did correlate with post-training perception of transfer $(r=.35)$, but in a weaker way than the research literature would suggest, and not by the commonly predicted factors.

Warr and Bunce (1995) investigated whether trainee characteristics such as motivation, self-efficacy, and learning task anxiety were related to learning scores, reactions to the training program, and changes in job behaviors within the context of an open learning program. The researchers gathered data from 106 trainees and their supervisors. The trainees were junior managers (93\% male) who were enrolled in a four month open-learning program.

The training was comprised of several modules delivered by four tutors from higher-education institutes. Trainees completed a questionnaire on their perceptions of 
their own motivation, self-efficacy and anxiety and mailed it to the researchers' university address. At the same time, the trainees' line managers rated the job performance of each trainee through a separate mailed questionnaire. Four months later, the trainees repeated the first questionnaire and program tutors submitted trainee grades from the first four months of training.

Warr and Bunce (1995) used hierarchical multiple regression analysis to analyze the data. The authors discovered that after all factors were entered, low age ( $b=-.25, p$ $<.05$ ), general attitude to training $(b=.24, p<.05)$, and analytic learning strategy ( $b=$ $.51, p<.001)$ accounted for a large proportion of the variance in learning scores $\left(R^{2}=\right.$ .49). The author's findings on pre-training motivation and its predictive power for posttraining results supports many other studies with similar findings.

Orpen (1999) conducted a study to determine the relationship between training environment and trainee motivation and perceived quality. The participants in this research were managers and employees employed by 12 different Australian private companies in the financial, retailing, and manufacturing sectors $(N=105)$. The average age of the participants was 30.6 years and they averaged 5.5 years tenure with their current employers. All participants attended at least one training event with their current employer prior to the study.

The independent variables in the study were the four personal aspects of the training environment: organizational commitment, job involvement, self esteem, and personal control, and four organizational aspects: social support - at work, social support - outside work, training incentives, training resources, and training needs. The dependent variables were trainee motivation and perceived training quality. Orpen (1999) utilized 
existing survey instruments which had been previously validated to measure the eight aspects of training environment. He found coefficient alpha scores for all scales ranging between .73 and .83 and therefore deemed all adequate for this research. Orpen performed separate correlations between each of the outcome measures and the eight scales.

With two exceptions the researcher found that all of the organization aspects correlated positively and significantly with both trainee motivation and perceived training quality $(p<.01)$. The only non-significant correlations were social support at work and social support outside of work against perceived training quality. The highest correlations were between training needs and the outcomes. With regard to the personal aspects, only organizational commitment correlated positively with perceived training quality at the .05 level. Orpen's (1999) findings suggest that trainee motivation seem to be more dependent on external, organizational climate factors than internal personal aspects.

From these findings Orpen (1999) asserts two major implications: (a) Organization leaders should focus more on the training environment if they want to improve trainee motivation and ultimately, training transfer, and (b) managers should provide the necessary time, money, equipment, facilities, and opportunities for training to be effectively implemented.

While the current research literature supports both self efficacy and motivation as being significantly correlated with transfer of training, there are relatively few research studies aimed at the underlying structures of these attributes. 
Machin and Fogarty (2004) conducted such a study focused on how transfer climate, and in particular, positive and negative affectivity relates to both pre and post-training aspects of training transfer.

The researchers selected trainees from the Queensland Police Service who attended training for a new computerized information system as subjects in the study ( $N=$ 137). Eighty-nine trainees completed a pre-training questionnaire and 104 trainees completed the post-training questionnaire. Forty-nine trainees completed the follow-up questionnaire. The researchers measured positive and negative affectivity using a 20item survey called the Positive and Negative Affectivity Survey (Watson, Clark, \& Tellegen, 1988). Ten of the items measured positive affectivity and included statements such as "I feel interested", and "I feel excited". Ten of the items measured negative affectivity and included statements such as "I feel distressed”, and "I feel hostile”.

Machin and Fogarty (2004) included measures for self-efficacy, motivation, and climate in the pre-training questionnaire. They measured pre-training self-efficacy using 12 items they developed specifically for the study. They measured pre-training motivation using nine items developed for the study. The researchers measured climate using the Climate for Transfer Questionnaire (56 items) based on Rouiller and Goldstein’s (1993) transfer climate factors. Finally, for the post-training questionnaire, Machin and Fogarty measured only one variable, transfer implementation intentions, using an eleven item survey developed for this study. Most scales produced Cronbach alpha reliability coefficients in the .80 to .94 range. There were two scales (negative reinforcement and positive reinforcement) which produced coefficient alpha scores of .66 and .79 respectively. 
Machin and Fogarty (2004) first used principle components analysis to check the dimensionality of the scales. They extracted two factors for the measure of pre-training self-efficacy, one factor for the measure of pre-training motivation, and three factors for the measure of transfer implementation intention. They used Bartlett's test of sphericity to show that the matrix formed by these variables was acceptable for factor analysis $\chi^{2}(15, N=104)=260.9, p<0.01$. The researchers then employed factor analysis to test the first hypothesis that two factors relating to antecedents and consequences was expected to emerge. Instead, they found that the factors grouped more into positive and negative valence groupings. In other words, participants were more influenced in their responses by the perceived positive and negative traits of the concepts versus the placement either before or after training.

Machin and Fogarty (2004) then regressed pre-training self-efficacy and pretraining motivation on positive and negative affectivity and the six climate transfer questionnaire (CTQ) variables. Test results showed that positive affectivity significantly contributed to the prediction of pre-training self efficacy ( $\beta=0.42, p<0.001 ; \beta=0.35, p$ $<0.01$ ). Negative affectivity also significantly predicted pre-training self efficacy and pre-training motivation $(\beta=-0.28, p<.01 ; \beta=-0.28, p<0.01)$. None of the CTQ variables were significant predictors of pre-training self efficacy. Only positive motivation was a significant predictor for pre-training motivation $(\beta=0.35, p<0.05)$.

Finally, for the third hypothesis, Machin and Fogarty (2004) regressed transfer implementation intentions on both positive and negative affectivity and the CTQ. Only negative affectivity $(\beta=-0.28, p<0.05)$ significantly predicted transfer intentions. None of the CTQ variables were significant predictors of transfer intentions. 
Implications from Machin and Fogarty (2004) are that training climate may not play as large a role in training transfer as suggested by other research. The study only focused on climate perception at the individual level and was thus limited by its scope. Other research has shown that department and organization level climate also play a large role in transfer effectiveness (Haccoun \& Saks, 1998).

While researchers have shown that some learner characteristics correlate to training transfer, there is mounting evidence that affective learner reaction to training does not. Ruona, Leimbach, Holton and Bates (2002) conducted a study to determine if utility learner reactions when compared to affective reactions explained variance in transfer. Affective reactions consist of items that portray participating satisfaction or liking of a course. Utility reactions signify the perceived work value of the training to the trainee. Ruona et al. used the utility items such as the following: My time was well spent, and, I would recommend this program to others in my organization.

The researchers surveyed 1,616 participants from a wide variety of organization and training programs with the Learning Transfer Survey Instrument. The sample included seven different organization types and nine different training types. Pearson product-moment correlations were used to test the association between participant utility reactions and predictors of learning transfer. Ruona et al. then tested whether the addition of utility responses added any predictive power after all the other transfer of learning factors derived from the LTSI instrument were accounted for using hierarchical multiple regression.

The researchers found the highest correlation with utility responses were transfer design, motivation to transfer, transfer effort, and perceived content validity, all having 
significant correlations higher than .450. In the regression test, despite being the final factor entered into the equation, participant utility reactions showed the strongest beta score of all factors entered into the equation $(\beta=.306, t=11.92, p<.01)$. The authors conclude that if participant reactions are to be used in evaluating training, they should only be utility reactions and not affective reactions.

In a related qualitative study, Yelon, Sheppard, and Sleight (2004) investigated how autonomous professionals form motivation to use new ideas gained from training. The participants were from all fifty states and Puerto Rico and participated in a yearlong training program $(\mathrm{N}=73)$. There were 32 males and 41 females. The researchers asked each participant four specific questions about their intentions to use the ideas they gained from the training and three questions about what ideas the participants actually applied in the work setting.

Yelon et al. (2004) used standard qualitative analysis methods and coding protocols to classify elements of the responses from the participants. After coding the set of responses, the researchers checked interrater agreement by having the primary researcher and another researcher practice rating one participant's responses before coding another five participants. They grouped the coding categories into four groups: (a) when participants gained the intention to apply, (b) what ideas they applied, (c) why they intended to apply ideas, and (4) how they applied the ideas. The percentage of agreement was $71 \%$ on the first two categories, $80 \%$ for the reasons given, and $96 \%$ on how participants applied the ideas.

The researchers found that in general the participant responses supported the idea that people mix their memories and momentary observations of job, task, and goals with 
the perception of training variables of ideas, examples, practice, and learning, to form specific decision criteria about future use. Motivation to transfer training is then a composite of how we think about or remember our jobs and our individual perception of training variables.

\section{$\underline{\text { Situational Characteristics that Influence Training Transfer }}$}

More than any other factor, the work environment can be either an enabler or barrier to training transfer in the workplace. A large amount of research over the past 15 years points practitioners to look at the environment first when assessing training transfer problems. Some research suggests that work environment accounts for $42 \%$ of the identified inhibiting factors in the transfer process (Foxon, 1993). Within the context of organization climate, we will discuss both job support and organizational support factors since they are often found in closely linked in both research and the minds of employees. Research findings on managerial support will follow the organizational climate discussion.

\section{Organization Climate and Job Support}

Organizational climate in the training environment is defined as the implied restraints to do a trained task and the perceived social support for training (Facteau, 1995). In addition to motivation and self-efficacy factors, a participant must judge the environment within which the learned skill must be used. Creating an organizational climate conducive to transferring and retaining learned tasks and knowledge is a key component to learning transfer. 
Rouiller and Goldstein (1993) investigated the influence of organizational climate on transfer of training. The authors conducted the study in a large franchise food chain's management development program. Participants $(N=102)$ attended training as part of a program to become assistant managers. The training program lasted nine weeks and covered all aspects of managing a quick service restaurant. At the completion of the training, company management assigned each of the 102 participants to 102 separate store units that required an assistant manager. Two weeks prior to the arrival of the new assistant managers, the researchers collected responses to an organizational climate survey from existing managers in the stores. Rouiller and Goldstein constructed the climate survey based on research literature, critical incident recording and focus group interviews. Though inferred, the authors did not list reliability measures.

In addition to the climate survey, the researchers collected data on four different measures: learning, transfer behavior, job performance, and unit performance. Rouiller and Goldstein (1993) performed a multiple regression to test the relationships between the variables and the climate survey responses. As predicted, they found that learning in training is significantly related to transfer behavior $F(1,100)=8.26, p<.01$. They also found that learning and organizational climate together account for $54 \%$ of the variance in transfer behavior $F(2,99)=3.58, p>.05$. Rouiller and Goldstein also investigated the relationship between learning and organizational climate, but found no significant results.

This study provided support for the assertion that transfer of training is affected by the organizational climate above and beyond actual learning. There are considerable implications for organizations and training vendors in this research; the vast majority of funds spent on training are usually directed to the learning and products that support it, 
but a very significant factor in whether the training is ever used is found within the climate that surrounds the training event.

Tracey, Tannenbaum, and Kavanagh (1995) conducted a study investigating the influence of the work environment on the transfer of supervisory skills. Participants were supermarket managers from 52 stores $(N=505)$. The researchers hypothesized that there was a direct relationship between transfer of training climate and post-training behaviors. The study was based on the aforementioned work by Rouiller and Goldstein.

For the final sample, 104 manager trainees, 104 supervisors, and 297 coworkers participated in the study. The average age of the trainee sample was 32 years and 57\% were women. The trainees had been with the company an average of eight years and had three years tenure in their current jobs.

The trainees attended a 3-day voluntary program on basic supervisory behaviors and skills. The training included topics such as interpersonal skills, customer and employee relations, and various administrative procedures. Multiple training delivery methods were used throughout the course, including role plays, audiovisual techniques, and lectures.

Manager trainees and their supervisors completed a survey of supervisory behaviors three weeks prior to the training. The trainees then attended the class over the next five months and completed pre- and post-learning assessments as supervisory knowledge measures. At the end of the training, trainees completed a transfer of training climate and continuous-learning culture questionnaire. Approximately six to eight weeks after training each trainee and his or her manager completed a post-training behavior questionnaire. 
Tracey et al. (1995) found a significant difference between pre-test and post-test knowledge scores $(t(103)=28.86, p<.01)$. Pre-test and post-test behavior was also significantly different $(t(103)=17.60, p<.01)$. The authors conducted a factor analysis on the transfer of training climate and continuous learning culture items. Principal components analysis using varimax rotation yielded a nine-factor solution that accounted for $68 \%$ of the variance. The authors found moderate to high correlations both between and among culture and climate surveys (.46 to .70) indicating that transfer of training climate may be better represented by less than nine factors.

Finally, to test the hypotheses about climate and transfer of training, Tracey et al. performed a structural equation analysis. The highest path coefficient for transfer of training climate was the social and goal cues indicator $(.90, p<.01)$. The highest path coefficient for the continuous learning culture was the social support indicator $(.91, p<$ .01). The strong correlations in these indicators support Rouiller and Goldstein’s (1993) work and lend further support to the importance of organizational climate in improving transfer of training.

Brown and Leigh (1996) conducted a study investigating employee perceptions of the organizational environment and how it is related to job involvement, effort, and performance. The authors hypothesized that psychological climate influenced job involvement, which influenced effort, which in turn, influenced performance.

The researchers collected data from two independent samples of salespeople. Sample 1 included sales representatives from a paper goods manufacturer $(n=77)$ and two office supplies manufacturing companies $(n=85$ and $n=16)$. Sample 2 included sales representatives of a large medical products company $(n=161)$. 
Brown and Leigh (1996) used confirmatory factory analysis to reduce a 22-item measure of psychological climate survey to six factors used in the study. Participants also completed surveys on job involvement and effort. The authors used manager ratings to assess on the job performance of the participants.

Using structural equation modeling, Brown and Leigh (1996) established a moderate relationship between psychological climate and job involvement $(r=.362)$ and an even stronger relationship between job involvement and effort $(r=.432)$. The relationship between effort and performance was also moderate $(r=.354)$. The researchers concluded that effort moderated the relationship between job involvement and performance. The results of the study imply that employees’ perception of their involvement in the job and subsequent effort have a relationship to their ultimate performance.

Seyler (1998) examined the link between climate and environmental factors surrounding training transfer and the motivation to transfer. The researchers surveyed employees from a large petrochemical company $(N=88)$ involved in a computer training course using 12 sub-scales derived from factor analyses of other validated instruments. Sub-scales measured desire to learn, internal work motivation, organizational commitment, computer confidence, training attitudes, reaction to the learning environment, reaction to content validity, supervisor support, supervisor sanctions, peer support, and opportunity to use.

Using hierarchical regression analysis for the dependent variable motivation to transfer, Seyler et al. (1998) showed that environmental factors such as opportunity to use and peer support influenced motivation more than any other set of variables and 
explained over $26 \%$ of the variance in motivation to transfer $\left(R^{2}=.625\right.$, adjusted $R^{2}=$ $.527)$.

While not specifically focusing on individual training transfer, organizational climate studies that focus on whole firm improvement are related in that prior studies have linked climate with motivation to learn. Delaney and Huselid (1996) investigated the relationship between human resource management practices and the perception of firm performance. The researchers sampled 1,127 organizations derived from the National Organization Survey (NOS), and asked respondents by phone to participate in a survey addressing organizational human resource management policies and practices. Representatives of 727 (64\%) completed either a telephone survey or a questionnaire survey. The NOS survey gathered perceptual data on human resource management practices and perceptual indicators of organizational performance.

The two dependent variables in the study were the first eleven questions from the NOS survey. Delaney and Huselid (1996) constructed the first variable from the first seven items assessing perceived organizational performance and created the second variable from the next four items assessing perceived market performance. Participants provided their scores for each dependent variable by answering Likert-type scales for each item $(1=$ worse, $4=$ much better $)$. The independent variables were the organizational HRM practices such as training, incentive compensation, and hierarchy.

Delaney and Huselid (1996) used regression analysis to test the relationships and reported that no independent variable had a high enough R2 value when combined with other variables in the regression equation to influence the outcome of perceived organizational or market performance in a meaningful way. They concluded that 1) 
HRM practices should never be isolated and measured to determine organizational effect. They always had larger coefficients when entered individually vs. with a group of other HRM practices; 2) the complexity of how HRM practices work together to form a climate requires the integration of micro-level and macro-level conceptual frameworks, strategy, and leadership in order to really understand what is happening in the culture, and 3) as more studies are conducted on the relationship between HRM practices and organizational performance, researchers must come to consensus on how HRM will be measured because there are no two studies that measure HRM practices in the same way.

In a similar study related to HRM practices, Pate (2000) conducted an investigation to understand whether a company's investment in HRD programs of lifelong learning pay off in terms of knowledge transfer and more positive employee perceptions of the company. The researcher conducted the study within a Scottish subsidiary of a major U.S. multinational company. Pate et al. surveyed both a sample group and a control group ( $n=114$ in each group). The sample group experienced an Education-for-all (EFA) program over the previous eight years. The EFA program provided employees with a broad focus on education rather than training in skills and development for a selected few. Respondents in both groups were very close in gender (85\% male). The sample group age range was 36-40. The control group age range was 31-35.

Pate et al. (2000) performed $t$-tests between matched pairs of questions for both sample and control groups. The independent variables were the two conditions of the treatment (EFA) and control. The dependent variables were response levels on each question. The impact of EFA on job satisfaction was both significant and positive 
$(t=-5.09, p<0.001)$. Participants in the EFA group did not, however, feel that the learning climate generated by the program created a stronger transfer climate. They did not feel that EFA created a stronger climate for manager support of training transfer. While the author recognized the limitation of this study as a single, atypical company case, Pate et al. asserted that further study of training transfer as it is supported by general learning climate enhancement initiatives is merited.

Taylor (2000) investigated the common types of transfer strategies used by key stakeholders within the context of workplace literacy training. In this qualitative study, the author selected 11 workplace literacy programs based on 4 pre-determined criteria: (a) how transfer of learning occurred in their specific program, (b) evidence that the program had been existence for over a year, (c) evidence that strong links had been developed with the shop floor supervisor of the company needing the service, and (d) evidence that the program was using some part of its curriculum to help trainees improve tasks or skills back on the job.

Taylor (2000) recruited participants from three different types of stakeholders: the instructor, the trainee, and the supervisor $(N=90)$. He then scheduled structured interviews with the participants based on Broad and Newstrom's Role and Time model of Transfer of Learning (Broad \& Newstrom, 1992). The Role and Time Model is a ninecell construct that examines the before, during, and after training influence and perceptions of the instructor, trainee, and supervisor. Taylor conducted the interviews for periods ranging from 25 to 50 minutes. He analyzed the raw data using NUD.IST qualitative software. 
Taylor (2000) found that the highest frequency of training transfer activities mentioned by participants was what the instructor did first during the training session and secondly, before the training session. Participants did not report supervisor activity as being high in frequency compared to either participant or instructor activity. Taylor did not attribute this difference to supervisors being less important in the transfer process, but rather, because their work environment created barriers to their participation.

The author identified several barriers to training transfer which included time pressures, peer pressures, limited opportunities to practice, and learner attitude. Taylor (2000) stressed that an organizational climate characterized by the pressure for participants to return quickly to their jobs after training was a major barrier to transfer. Supervisors also identified that a lack of time to coach and follow-up with their employees as a barrier. Taylor's research supports other similar research focused on barriers to transfer.

Clarke (2002) investigated work environment factors which influenced training transfer within a human service agency. In this qualitative study, Clarke conducted semistructured interviews six months after trainees $(N=14)$ from a United Kingdom social services agency attended two-day service training. The researcher's focus in this study was to examine which factors influenced the transfer of training within a social services organization and how these factors compared to those discussed in the wider training literature. He also collected quantitative data which were reported in another study. The qualitative feedback corroborated the findings from the quantitative data, indicating that there was minimal transfer of the training back to the work place. 
Clarke (2002) addressed four major content areas in each interview: (a) trainee’s views regarding the benefits they perceived they had obtained as a result of attending the training; (b) evidence of utilizing the training in practice; (c) barriers to implementing the training; and (d) factors which assisted with either learning or training transfer. The researcher's primary finding from the interviews was that job and work environment factors impeded the use of training on the job. Eleven of the 14 trainees identified the short duration of the training program as a primary deterrent and that any skill obtained in training was undermined by a lack of practice back on the job.

Many of the trainees also indicated a lack of reinforcement from supervisors and peers for their new skills back on the job. When trainees did experience support from supervisors, it was usually broad and general in nature, without focusing on specific skills for enhancement or improvement. Clarke (2002) cited other studies where supervisors did provide follow-up feedback and encouragement that was specific to the training. In these cases participants cited more frequent usage of the trained skills back on the job. Finally, Clarke found it surprising that eleven of the fourteen participants felt that the training was targeted more for personal development rather than a job skill they could use in their workplace. They found this created an obstacle to their learning for the intended purpose. The researcher suggests that the results of this study support Baldwin and Ford's transfer model (Baldwin \& Ford, 1988). He cautioned that the study should probably not be generalized beyond the small group representing the service industry in the UK.

Montesino (2002) explored the relationship between transfer of training and corporate strategy as an external influence. His research question was whether 
employees and their supervisors engaged in transfer-enhancing activities based on their perceptions of the organization's goals and strategies. To answer this question, Montesino selected a population of 250 sales representatives from a large mid-western pharmaceutical company who participated in a targeted sales training program and the supervisors who managed them. He mailed a survey to a sub-sample of both employees ( $n=180)$ and supervisors $(n=50)$. One hundred forty-seven sales representatives and thirty-six supervisors responded with completed surveys.

Montesino (2002) administered a pilot test of the questionnaire prior to conducting the survey. He established validity of the four indexes making up the survey by obtaining standardized alpha reliability coefficients of .88, .71, .88, and .96. He administered two versions of the questionnaire: one for sales representatives and one for their managers.

Montesino (2002) investigated the following variables: (a) perceived presence of practices to support usage of training, (b) perceived alignment of training with the strategic direction of the organization, (c) awareness of the strategic direction of the organization, (d) commitment to the strategic direction of the organization, and (e) selfreported usage of training. Survey questions representing each variable were drawn from a variety of researchers' prior work with alpha coefficients in the .88 to .96 range.

Montesino (2002) tested the data using a one-tailed Spearman rank order correlation coefficient for trainees’ data and a Pearson product-moment correlation coefficient for field managers, both at the 0.05 alpha level. He found that for both trainees and managers there was a significant correlation between the variables "perceived presence of practices to support usage of training” and "perceived alignment 
of training with the strategic direction of the organization" (trainees: $r=.29, p<.001$, managers: $r=.38, p<.03)$. He thus, rejected the null hypotheses and asserted there was a low positive relationship between the variables. Montesino also conducted MannWhitney $U$-tests to understand the relationship between the variables with ordinal data. These also confirmed the findings of the prior tests in showing that there is a low to moderate correlation between perceived alignment of training with the strategic direction of the organization and the presence of training transfer practices.

Despite, given the industry, the limited generalization that can be gleaned from such a narrow study, Montesino’s (2002) findings provide important directional information about how training and training dollars can be utilized more effectively outside of the usual and customary focus on training design and delivery.

Training researchers have focused much of their efforts on either organizational learning cultures or individual discrete learning methodologies. There is very little research literature combining these two disciplines. Egan, Yang, and Bartlett (2004) however, investigated the relationship between organization's learning culture and job satisfaction with employee's motivation to transfer training. They hypothesized that a learning culture would mediate job satisfaction and both in turn would mediate both the motivation to transfer learning and turnover intention.

To test this theory, Egan (2004) conducted a survey of IT workers in 50 large corporations (over 500 employees). The researchers received 245 completed surveys from 13 , or $26 \%$ of the firms, but the actual participation rate for each firm was unknowable because the total number of IT workers and how many came from which firm were not included in the design of the study at the request of the participating firms. 
Egan et al. (2004) used the abbreviated form of the Dimensions of Learning Organization Questionnaire (DLOQ) developed by Watkins and Marsick (Watkins, 2003) to assess organization learning culture. The abbreviated form of the DLOQ contains three dimensions (reduced from seven) and seven items for each, totaling 21 items (Yang, 2003). The authors used confirmatory factor analysis which supported a satisfactory fit between the seven dimension model and their data, $\chi^{2}(165)=437.18, p<.01$. The Cronbach's alpha for the seven dimensions were .71, .83, .83, .74, .86, .83, and .90, and reached as high as .95 for the twenty-one item scale reliability estimate. Egan et al. selected one item from each of the seven dimensions to assess the construct of learning culture with a Cronbach alpha estimate of .89. This created a single construct for organization learning.

The researchers selected five items to assess motivation to transfer that had been used and validated previously by researchers in the field. These five items exhibited Cronbach alpha estimate of .83 and were considered reliable. Previous research contained all five items. Egan et al. (2004) selected three items to measure turnover intention which were adapted from studies by Irving, Coleman, and Cooper (Irving, Coleman, \& Cooper, 1997). The authors felt that although coefficient alpha scores were somewhat lower, at .68, they were still within marginally acceptable limits. Egan et al. used three items from the Michigan Organizational Assessment Questionnaire to assess job satisfaction (Cammann, Fichman, Jenkins, \& Klesh, 1979). The questionnaires contained seven-point Likert scales and exhibited Coefficient alpha estimates which were moderate, at .70. 
The researchers used structural equation modeling to evaluate the relationships between the study factors. Results of the study showed that the organizational learning culture had a significantly positive contribution on job satisfaction with a structural path correlation (SPC) of .68. Organizational learning was also positively and significantly correlated to motivation to transfer learning (SPC $=.28$ ). With regard to turnover intention, Egan et al. (2004) found that while organizational learning culture had a significant impact on job satisfaction, and that in turn job satisfaction correlated significantly, and negatively with turnover intention $(\mathrm{SPC}=-.43)$, organization learning culture did not have a strong and significant negative correlation with turnover intention (SPC $=-.16)$. In other words, in this study and within this sample, the researchers found that creating a learning organization culture, while a moderating variable for job satisfaction, had very little to do with whether an employee had an intention to leave the organization.

The type of organization, culture, and transfer system also appear to have a different influence on transfer rates. Holton, Chen, and Naquin (2003) conducted a study to examine the differences in transfer systems across eight organizations, three organizational types, and nine training types. The researchers used purposive sampling from an existing database of 4,562 responses to the Learning Transfer System Inventory (LTSI) and selected 1,099 individuals employed by eight different U.S. organizations. The organizations represented four private sector companies, three public sector agencies and one nonprofit organization.

The researchers examined the following questions: Are there significant differences in transfer system characteristics between organizational types (profit, 
nonprofit, and public sector)? Are there differences in transfer system characteristics between specific organizations? Are there differences in transfer system characteristics between different training types?

Holton et al. (2003) used MANOVA to answer the three research questions because the questions involved multiple dependent variables. The sixteen constructs of the LTSI were the dependent variables. Results showed statistically significant differences on all criteria for Question 1, suggesting that transfer system characteristics differed across organizational types. Performance outcomes Expectations, Opportunity to Use, and Personal Capacity for Transfer were significantly higher for private organizations compared to public firms. Supervisor sanctions, Resistance to Change, and Personal Outcomes Negative scales were significantly higher in public organizations than in private ones.

When looking across all organizations, one organization had a significantly different transfer environment than the other seven. In five of seven work environment associated scales, this organization was significantly higher than at least two others. This suggests that each organization has its own unique positive and negative transfer factors.

Finally, Holton et al. (2003) found that transfer systems are significantly different across training types. For example, participants who received sales training rated Motivation to Transfer, Performance Outcomes Expectations, and Opportunity to Use significantly higher than those who received new employee academy training. These results point to a growing theme in training transfer research that one model of transfer may not fit all and that organizations need to tailor their systems to the specific culture, environment, and industry in which they work. 


\section{Managerial Support}

Until recently, perceived level of manager and supervisor support has received relatively little focus from researchers investigating transfer factors (Baldwin \& Ford, 1988). If training is not reinforced by what management is saying and doing everyday, there is little chance for it to succeed by any measure. Over the past 10 years, practitioners and researchers have become increasingly aware that the environment that a trainee returns to is at least as important as the one in which they received formal instruction (Broad \& Newstrom, 1992).

Huczynski and Lewis (1980) conducted one of the earliest studies investigating supervisory influence on transfer of training. The participants in the study came from two groups. The first group was comprised of participants enrolled in an 'open’ network analysis course run by Glasgow University $(n=17)$. This group was known as the University group. The second group came from within a single large Scottish electronics company enrolled in an in-house network analysis course $(n=32)$. This group was known as the Company group.

The researchers used qualitative techniques (structured interviews) and descriptive statistics as methodology for this study. They found that of the forty-eight studied, seventeen (35\%) attempted to transfer what they had learned from the course to their work. In subsequent interviews, Huczynski and Lewis (1980) found that more than twice the number of experimenters had discussed the content of the course with their supervisors before the course compared with those who did not experiment with transfer. 
Huczynski and Lewis (1980) found in subsequent interviews with participants that those who had not discussed the course with their supervisors prior to attending had no clear understanding of why they were attending. They also found that participants who had discussions with their supervisors had a much clearer understanding and purpose for being there.

When asked about inhibiting and facilitating factors the researchers found that supervisors influenced transfer by using facilitating methods (openness, listening skills, empowerment), but could also weaken transfer by using inhibitors (work overload, unplanned work, high rate of change). Huczynski and Lewis (1980) suggest from these findings that a supervisor's influence can work either negatively or positively with respect to transfer results. The researchers suggest that for transfer to occur, each of the four major transfer factors of student, supervisor, facilitator, and organizational context must be present in order for transfer to take place, and that all are within the sphere of management influence.

Becker and Klimoski (1989) examined the relationship between the perceived organizational and supervisory feedback environment and performance. The researchers asked the primary questions (1) What sources of feedback are related to performance, and do any sources seem to be more related to performance than others? and (2) What types of feedback from the various sources are related to performance?

The authors mailed the presidents of 20 firms sourced from a metropolitan business directory a brief letter of introduction and request to participate in the study. One manufacturing firm agreed to participate in the study. Becker and Klimoski (1989) distributed questionnaires to 152 employees of the organization. Ninety-seven 
participants returned the surveys for a response rate of $63.8 \%$. Participants ranged in age from 21 to 67 years with an average of 41.5 . Females comprised $41.7 \%$ of the participants and $88.5 \%$ were white.

The researchers used the Job Feedback Survey as the primary measurement tool in this study. It contained 15 dimensions with each item scored on a Likert-type scale (1 $=$ Never to $5=$ Very Often). The survey produced both an overall positive feedback score and an overall negative feedback score. Supervisors, job incumbents, and peers provided performance measures for the study, rating each participant on a 12-point scale for quantity and quality of work, attitude, cooperation, communication and overall performance.

Identifying which of the six overall feedback dimensions (i.e., positive and negative feedback from supervisors, incumbents, or peers) was the overall goal of the study. Using simultaneous multiple regression, the researchers found that the composite measure of performance regressed significantly on the supervisory factors $\left(R^{2}=.46\right.$, $F(5,90)=4.80, p<.001)$. Becker and Klimoski (1989) concluded that positive feedback from the supervisor is related to higher performance and negative feedback from the supervisor is related to lower performance. These findings support other similar research in supervisory influence and build a foundation for supervisory training transfer disussions.

Brinkerhoff and Montesino (1995) conducted a study exploring the impact of pretraining supervisor expectation discussions and post-training supervisor follow-up discussions with participants in a management training program. They hypothesized that there would be a positive significant relationship between management support for 
training and transfer of training for participants when compared against other participants who did not receive management support. The researchers selected employees from a Michigan-based Fortune 200 pharmaceutical company who happened to enroll in one of five corporate training courses during the study time frame $(N=91)$.

Of the 91 participants, approximately one-third were randomly selected from each class as the experimental group $(n=37)$. The researchers informed the experimental group's supervisors how they would participate in the study, but did not inform remaining class participants' supervisors about the study. In this manner, Brinkerhoff and Montesino (1995) divided each class into two groups: (a) the experimental group, who’s supervisors were asked to provide a brief before and after training discussion, and (b) the remaining group, who's supervisors were not asked to do this. The research team distributed a survey approximately ninety-days after the training ended in order to measure the number and degree of transfer behaviors and the frequency of meetings between participants and their supervisors.

Brinkerhoff and Montesino (1995) used the t-test for independent means with and alpha level of .05 to determine if there was a difference between the mean transfer scores of the two groups. The researchers found there was a significant difference between the two groups (experimental: $t(37)=3.34$, comparison: $t(54)=2.88, p<.05$ ), and thus rejected the null hypothesis. The researchers then formed two new groups based on how the participants scored on the transfer scale. They then compared how the high transfer scoring group did when compared to the low transfer scoring group when compared against three survey items depicting how managers showed support. In each case, the 
high transfer scoring group produced averages significantly higher than the low transfer scoring group on these items.

Brinkerhoff and Montesino's (1995) research supports many other studies done in the early stages of transfer research showing the critical importance of manager involvement and support both during and after training. There are some exceptions, however, to these studies and the training transfer literature offers moderate disagreement on how much influence supervisors wield in improving transfer among their direct reports.

Xiao (1996) conducted a study within the Chinese electronics industry to investigate the relative strength of organizational factors on transfer of training. The participants in the study came from one of four companies representative of the industry $(N=106)$. Xiao developed a survey that measured five areas deemed to influence training transfer: orientation, knowledge and skill acquisition, rewards, supervision and peer relationship. The researcher designated these areas as independent or predictor variables in the study. The dependent variable in the study was perceived output of transfer behavior.

Xiao (1996) measured validity and reliability of the survey using Cronbach’s alpha and inter-scale correlation. Cronbach's alpha scores ranged from .58 to .83. Xiao determined inter-scale reliability for each scale by comparing the intra-scales' reliability estimates with the inter-scale correlations. All scale inter-scale correlations were higher than their intra scale correlations, and therefore judged to be adequate.

After data collection, Xiao (1996) tested the assumed relationship between the organizational factors and transfer of training by using hierarchical regression analysis. 
Xiao first loaded the type of company variable (state-owned and joint venture), followed by worker characteristics, training achievements, application orientation, rewards, supervision, and finally peer relationship. The researcher found there was no difference in perception of transfer of training in the two types of companies $(\beta=-.0024, t=-.024$, $p>$.98). He also found little influence from the worker characteristics on perceived transfer of training $\left(R^{2}=.043, F(5,96)=.81, p>.55\right)$. Training achievement was significantly related to training transfer $\left(R^{2}=.175, F(6,95)=3.14, p<.0076\right)$ and accounted for 14 percent of the variance in transfer behavior (partial $R^{2}=.14$ ). The largest influence on training transfer was supervisor and peer support. When added to the equation, $R^{2}$ increased to $.374 F(11,90)=4.45, p<.0000$ and partial $R^{2}$ was .16 , suggesting that 16 percent of the variance in transfer could be attributed to supervisor and peer support. Examined individually, the B value for supervision was .27 ( $t=2.45, p<$ $.016)$, and the B value for peer support was $.19(T=1.96, p<.056)$. From these tests, Xiao concluded that supervision had a significant partial contribution to transfer of training as perceived by participants.

Bates, Holton, Seyler, and Carvalho (2000) conducted a study to measure the impact of training content validity, opportunity to use learning, and interpersonal support factors on supervisory ratings of employees' application of operating procedures. The employees learned the procedures from a computer-based training program. Participants in this study were operators from a highly hazardous chemical manufacturing facility ( $N$ =73). Operators participated in training and certification on up to 150 CBT training modules. 
Following the modules, the participants completed two survey questionnaires: The Transfer Climate instrument (36 items) and the Reaction to Training Questionnaire (21 items). For each survey instrument, a 5-point Likert-type scale (1 = Strongly Disagree, 5 = Strongly Agree) measured responses for all items. The researchers used factor analysis to reduce the surveys to six constructs with Coefficient alpha estimates ranging from .68 to .95 and an average alpha of .79. The following scales were used: supervisor support, supervisor sanctions, peer support, change resistance, opportunity to use, and content validity. Bates et. al (2000) used a combination of performance ratings and observations as the dependent variable.

The researchers used hierarchical regression analysis to test the data and found that only the supervisor support variables $\left(R^{2}=.18, F_{(2,60)}=6.93, p<.01\right)$ and the coworker support variables $\left(R^{2}=.20, F_{(5,60)}=3.57, p<.01\right)$ were significant in explaining a positive variance in the performance ratings. This finding supports previous research (Seyler et al. 1998) showing that interpersonal factors such as supervisor and peer support are significant predictors of training transfer.

Van der Klink, Gielen, and Nauta (2001) found that supervisors played a far less significant role than trainers, trainees and the training approach in the transfer process. Van der Klink et al. conducted an experimental study using two groups employed at a German bank. The researchers used Baldwin’s (1987) assumptions regarding supervisors setting behavioral goals for the application of the training content to trainees' jobs. Van der Klink et al. also employed Broad and Newstrom’s (1992) principles regarding supervisory support in the experimental treatment and hypothesized that a higher degree of supervisory involvement would result in higher rates of trainee job performance. 
The study included pre and post measurements for both experimental and control groups. Both groups received identical assistance from the trainer and developed action plans that addressed transfer intentions, barriers, and supervisory support after training. Supervisors for the experimental group alone, however, received letters from the training department prompting them to conduct discussions, action planning, and other transfer activities with their employees after the training. The researchers sent two questionnaires to both groups five weeks after completion of the training: one on supervisory support and one measuring trainee’s job performance.

Van der Klink et al. (2001) found that while the experimental group rated their supervisors significantly higher than the control group $(p<.05)$, the post training performance results between both groups did not differ significantly (Mann-Whitney test: mean rank comparison group 8.65, $n=10$, experimental group $10.65, n=8$, one tailed, $p<.23)$. Moreover, in the ongoing performance comparison, the control group performance was significantly higher than the experimental group (Mann-Whitney test: mean rank comparison group 11.35, $n=10$; mean rank experimental group 7.19, $n=8$, one tailed, $p<.05)$.

A large amount of recent research suggests that organizational climate, and more specifically, supervisory support within that climate is at least as important as the learning event in facilitating transfer. These studies underscore the need for further research and inquiry into the supervisor-subordinate relationship as it relates to training. 


\section{Training Design Considerations}

Much of the early literature on training transfer focused on training design characteristics. While this study is not focused on the design of training as a mediator for training transfer, it is important to consider two specific design features that have implications for both pre- and post-training supervisory support. Because supervisory support is one of the independent variables in this study, both goals setting and relapse training merit further discussion in this research.

Design characteristics are typically the mechanical and instructional processes through which training is conveyed and received in order to obtain a specified learning outcome. These characteristics are independent of the trainee, trainer, or supervisor in the training transfer process. Goal setting and relapse training, however, are design characteristics which often require supervisory support to accomplish.

\section{Goal Setting}

While there are many facets to instructional design, goal setting is one most often associated with training transfer effectiveness. Goal setting is the process of setting personal goals for post-training behavior based on the learning objectives of the training. These goals are often set with oversight and encouragement of supervisors or mentors.

Wexley and Baldwin (1986) explored the post-training strategies of assigned goal setting, participative goal setting and behavioral self-management approaches to enhancing training transfer. They selected 256 students (143 women and 113 men) enrolled in an upper-level management course at a large Midwestern university to participate in a three-hour training workshop on improving time management skills. 
Sixty subjects (33 women and $27 \mathrm{men}$ ) who were assigned the goal-setting condition, attended a one hour thirty minute transfer session two days after the time management workshop. During this session, the subjects received a list of 17 behavioral goals to be performed in the ensuing weeks.

Subjects in the participative goal-setting condition ( $n=65$, 41 women, 24 men), met for one hour thirty minutes two days after the workshop and were asked to consider their highest ranked learning points from the training session. The participants then, with the help of the facilitator, reduced their lists to a few behavior goals to be achieved in the coming weeks.

Subjects in the relapse-prevention condition ( $n=63,31$ women, 32 men) met for the one hour thirty minutes two days after the workshop and learned self-control strategies designed to help them avoid relapse in the future. A final control group subjects ( $n=68$, 38 women, 30 men) did not participate in any transfer session after the workshops.

Wexley and Baldwin (1986) collected dependent measures of reaction, learning, and behavioral changes at 3 different points: (a) after the workshop, (b) after the transfer sessions, and (c) after the eight-week study period. ANOVAs performed on the dependent measures for time 1 and time 2 revealed no significant differences across conditions with regard to reactions and learning. Wexley and Baldwin (1986) did find significance during time 3 across conditions with regard to learning $(F=4.25, p<.01)$ and the post/then behavioral self-report measure $(F=3.39, p<.02)$. Interestingly, multiple comparisons performed on the means of the significant main effects showed that the assigned goal-setting subjects scored significantly higher on learning during time 3 
than subjects did in the participative goal setting $(t=3.15, p<.02)$, relapse prevention ( $t$ $=3.05, p<.003)$, and control conditions $(t=3.15, p<.002)$. Wexley and Baldwin's research underscores the importance of assigned goal setting within the context of post training strategies to improve transfer effectiveness.

Richman-Hirsch (2001) replicated prior research by conducting a study to examine the effectiveness of goal setting and self-management as an effective posttraining intervention to enhance training transfer. Prior research in these areas found correlation for both activities to effectively transfer of training. In this study, 267 employees of a large mid-western university completed survey questionnaires on their colleagues who had recently completed a customer service skills training class. The two independent variables, goal-setting and self management were regressed against the dependent variables of maintenance and generalization. Findings indicated that goalsetting predicted both maintenance and generalization of training skills $(p<.05)$ while self management did not.

The interaction between post-training interventions (goal setting compared to self management) was significant in predicting maintenance $(b=.68, p<.01)$ and predicted generalization at a larger alpha level $(b=.86, p<.10)$. Richman-Hirsch found goal setting to be an effective mediator for training transfer.

In a study with similar implications, Werner, O’Leary-Kelley, Baldwin, and Wexley (1994) examined whether pre- and post- training interventions enhanced training outcomes. They selected 150 students from an undergraduate management course at a large Midwestern university to participate in assertiveness skills training conducted by the authors. The students averaged 21.5 years of age and were nearly equally divided 
between men and women ( $n=73$ and $n=77$, respectively). Werner et al. utilized a 2 x 2 factorial design where pre-training intervention (provided or not provided) was crossed with post-training intervention (provided or not provided). They assigned four conditions as Condition 1: no pre- or post-training intervention, Condition 2: pre-training, but no post-training intervention, Condition 3: post-training, but no pre-training intervention, and Condition 4: both pre- and post-training interventions.

Werner et al. (1994) conducted all training within a two-week period with students randomly assigned to a condition. During the pre-training session, the researchers pointed out how the students could use the training in their own work lives and pointed out individual opportunities for each to work on to become more assertive. They also discussed how the training could impact their future careers. During the posttraining session, the research team assigned students behavior goals and activities checklists which the students were to complete twice a week for four weeks.

Werner et al. (1994) collected five measures of training effectiveness: immediate reaction, delayed reaction, learning retention, behavioral reproduction, and behavioral generalization. The reaction measures were survey based. The other measures were observation based. Scores for all measures were collected and means were reported by condition.

The researchers conducted multivariate analysis (MANOVA) to test for overall differences between pre- and post-training interventions on the five measures. Werner et al. (1994) did find significance for the main effects of post-training interventions (Hotellings, $F(1,60)=7.31, p<.001$ ). Interestingly, they did not find significance for the main effects of pre-training interventions. For the learning retention measure, Werner 
et al. again found strong effects for the post-training intervention. The students who set goals in the post-training intervention could recall 1.5 times more learning points than those who did not have this treatment $F(1,146)=4.81, p<.001$, partial eta-squared $=$ .26. Those who were assigned the goal setting session, however, reported liking the training significantly less than those who were not assigned. Four weeks later, however, the students who received goal setting in the post-training treatment recalled more learning points than the students in the other conditions. From this study it appears that post-training interventions may be the bad tasting medicine that ultimately proves efficacious for learning results.

Goal setting is one strategy that will improve transfer of training results given the proper conditions. More research in this area in combination with supervisory feedback and support is warranted.

\section{Practice and Relapse Training}

Both practice and relapse training are training design strategies associated with what the learner does after the training occurs. When a learner practices the skills learned in training and subsequently returns for refresher or relapse training, training transfer is improved. Practice and relapse training are of concern in this study because they are often initiated by a supervisor for employees who need to improve or upgrade their skills. Without supervisor support, many employees may not choose to attend relapse training.

In an early training transfer study, Byham, Adams, and Kiggins (1976) investigated the technique of modeling and practice on training transfer. The researchers 
hypothesized that modeling and practicing training content would increase transfer over a control group that only received instruction. Participants were members of two accounting departments in a mid-western corporation's headquarters attending workshops covering different supervisory skill modules. The dependent variable was subordinates' perceptions of their supervisors' handling of coaching session interactions based on 10 focus areas. Examples of the focus areas included orienting a new employee, overcoming resistance to change, reducing tardiness, and delegating responsibility. The independent variable was the modeling treatment.

Byham et al. (1976) used an experimental design with descriptive statistics showing differences in perceived performance between pre and post assessments for both the control group and trained group. The researchers found that in all but one case, focus area scores improved from pre-test to post-test and from control group to trained group. Byham asserted that the results added credibility to the growing movement of modeling and practice for transfer of training.

Tziner and Haccoun (1991) conducted an investigation of relapse prevention (RP) training as a training transfer strategy. They hypothesized that participants in training that included RP strategies would show more positive reactions and a higher motivation to transfer. They also hypothesized that RP would result in higher outcome variable scores (reaction, learning, and skills).

Tziner and Haccoun (1991) selected military officers in the Israeli Defense Forces who had participated in the Advanced Training Methods program as participants in the study $(N=81)$. The participants ranged from 19 to 23 years old and were almost evenly split between genders (39 men, 42 women). The researchers assigned 45 of the officers 
to participate in a post-training relapse prevention module at the end of their regular training. The remaining 36 subjects served as the control group and did not participate in the RP training.

The control and treatment groups served as independent variables in this study. Dependent variables were measured by nine scales as follows: locus of control, environmental support, motivation to transfer, training reactions questionnaire, content mastery, use of trained skills, use of transfer strategies, use of trained skills, and use of transfer strategies. Tziner and Haccoun (1991) derived each of these scales from previous researchers’ work in their respective areas.

The research design was a 3 X 3 X 2 factorial ANOVA along with a stepwise discriminant analysis for the dependent variables. From the discriminant test, the researchers obtained an overall canonical correlation $\left(R_{\mathrm{C}}=.53\right)$ which was significant $(\mathrm{X} 2$ $=22.39, p<.01$ ) The ANOVA showed that the RP group was significantly higher on four of the outcome variables (content mastery, self-report use of transfer strategies, immediate supervisor evaluation of use of trained skills and training transfer strategies). Additionally, RP participants showed significantly higher levels of knowledge of course content when compared with non-RP participants $F(1,80)=10.24, p<.01$. The same RP participants also reported using the transfer strategies taught in the course with more frequency $F(1,80)=6.18, p<.05$, eta-squared $=.08$. Finally, Tziner et al. found significance in the three-way interaction, indicating that trainees that perceived a more supportive environment and who had received the RP treatment, were perceived as displaying greater levels of transfer strategies by their supervisors. The authors cite 
limitations in the training area and industry of this case, but believe there is strong support to conduct further studies in relapse prevention training because of their findings. Another form of relapse prevention training is the opportunity a trainee has to perform a learned task on the job. Ford, Quinones, Sego, and Sorra (Ford, Quinones, Sego, \& Sorra, 1992) investigated practice opportunity after training and how it is affected by supervisory attitudes, workgroup support, self-efficacy, and cognitive ability.

The participants in the study were Air Force Aerospace Ground Equipment personnel who graduated from a technical training course. Ford et al. sent surveys to a matched sample of airmen and their supervisors $(N=180)$ obtaining a $57.1 \%$ response rate. The majority of the airmen and supervisors in the sample were male (92\% and 95\% respectively). The predictor or independent variables in the equation were command (type of base), supervisor attitudes, and self efficacy. The dependent variables were breadth of activities required on the job, activity level of tasks on the job, and type of task performed on the job. Ford et al. (1992) employed a hierarchical regression analysis as the primary statistical test in this study.

The researchers found that work context factors were significant predictors for breadth of training, with beta-weights showing supervisory perceptions as the primary influencer $\left(R^{2}=.10, p<.05\right)$. In like manner, self efficacy accounted for a significant increment in the amount of variance accounted for in breadth (change in $R^{2}=.10, p<$ .05). With regard to activity levels, ability had a significant positive influence (change in $\left.R^{2}=.04, p<.05\right)$. Airmen with higher ability levels performed tasks they learned in training more often. Finally, supervisory perceptions and peer support were significant predictors of task type (change in $R^{2}=.47, p<.01$ ). In other words, airmen reported they 
performed more difficult tasks as their peer and supervisor support increased. Ford's findings further support the hypothesis that opportunities to perform trained tasks after training improve perceived levels of transfer.

In focusing on the training environment, Burke and Baldwin (1996) conducted a study to determine whether relapse prevention training and the training climate itself had an affect on training transfer. The researchers selected employees $(N=78)$ from a Midwestern firm who would attend a supervisory skills curriculum. In the training, participants learned basic coaching and feedback skills. After the training module, Burke and Baldwin assigned the participants to one of three experimental conditions: full relapse prevention (RP) training ( $n=28)$, modified RP training $(n=27)$, or no RP training $(n=23)$.

Relapse prevention training is based on Marx’s (1986) model that seven steps participants should follow to maximize training retention and transfer through time. These steps include goals setting for the learned skill, anticipating obstacles, and determining positive and negative consequences for either using or not using the skill.

Trainees completed a survey immediately following the module. Responses to all survey items were based on a 7-point Likert scale (1 = strongly disagree, 7 = strongly agree). Burke and Baldwin (1996) used the surveys to measure transfer climate at both the individual and subunit level. Four weeks after the course, the researchers collected learning measure data, or dependent variables, which were trainee retention, use of transfer strategies, pre-class skill usage, and number of coaching sessions conducted. The independent variables were the conditions of relapse training and no-relapse training. Chronbach alpha levels for all measures were acceptable and above 0.7. Inter-rater 
reliability between learning measures was $86.4 \%$. A factor analysis on the climate measures indicated that three of eight measures generated factors accounting for $63.2 \%$ of the variance. The three underlying dimensions were labeled as immediate supervisory influence, attitudes toward training, and training practices and policies.

Burke and Baldwin (1996) a conducted a four-stage hierarchical regression and found that neither full nor modified RP training had a significant main effect on any of the maintenance outcomes. Transfer climate, however, did show an interaction effect between relapse training and training climate $\left(R^{2}=.19, p<.05\right)$. In other words, trainees who faced a more favorable transfer climate were more likely to use relapse prevention techniques. The regression also showed that full relapse prevention training resulted in better training outcomes in less supportive environments while modified relapse training resulted in better outcomes in fully supportive environments. Burke and Baldwin’s research underscore the importance of considering the training climate within which any transfer strategy is attempted.

May and Kahnweiler (2000) compared mastery practice design to conventional behavior modeling workshop practices to test learning and transfer differences. Participants in the study were first-line supervisors and managers working in a manufacturing plant located near Georgia $(N=38)$. Men outnumbered women in this convenience sample nearly two to one (26 men, 12 women).

All participants attended a 4-hour listening skills workshop and were then divided into two groups of matched pairs by coin flip. May and Kahnweiler (2000) assigned one group to master lab practice (treatment) and one group to conventional workshop practice (control group). The dependent variables were knowledge retention, behavior skill 
demonstration, and transfer to the job. The independent variables were the two treatment conditions.

The research design was correlation tests between the dependent variables and one-way ANCOVA to test differences between the independent treatments and dependent variables. May et al. observed significant correlation between the behavioral demonstration and knowledge retention measures $(r=.34, p<.05)$. He did not find significant correlation between either retention or behavioral demonstration and the transfer measure $(r=-.09, p>.05 ; r=.01, p>.05)$. The researchers then conducted a one-way analysis of covariance on each dependent measure to test whether the posttraining results for the two groups differed after adjustments were made for pre-training differences. May and Kahnweiler (2000) found that the mean retention scores for the mastery practice group were significantly higher than the retention scores for the conventional practice group, $F(.033,1,35)=6.06, p<.019$. He found the effect sized to be relatively large at .40 (Cohen, 1977). In a similar manner, the behavioral demonstration means for the mastery practice group were significantly higher than the demonstration scores for the conventional practice group $F(.033,1,35) 5.39, p<.026$. The effect size was .38, which is a medium effect size according to Cohen. May and Kahnweiler found no difference between the mastery and conventional groups on the far transfer measures.

May and Kahnweiler (2000) concluded that mastery practice did aid the transfer of training immediately following the training event. Results diminished however, as time passed. While the generalizability of the research has limitations due to the small number of subjects and unique experimental conditions of the study, May points to the 
relevancy of the study in pointing future research toward a greater understanding of mastery practice and far transfer.

May and other researchers have developed new promising avenues for transfer research in the future. Practice and relapse training, in combination with other transfer factors are beginning to form a research body that points to a system of strategies rather than a single causal relationship for learning.

\section{Transfer Measurement Instruments}

Holton and Bates (2000) investigated and developed what has become a widely used instrument to measure transfer behavior within an organization. The author developed the Learning Transfer System Inventory (LTSI) and administered it to a large number of participants from a wide range of organizations. The LTSI is included in this review because there is a very large body of research that contains this instrument as its primary measurement device.

The researchers administered the survey to four categories of organizations: government, for-profit, nonprofit, and public training classes ( $n=676,432$, 192, and 316 respectively). The subjects were participants in one of eight categories of training classes: technical skills, sales/customer service/leadership/management, professional skills, supervisory skills, clerical, communication, and computer.

Holton and Bates (2000) utilized common factor analysis with oblique rotation to consolidate 112 items into nine constructs: supervisor support, opportunity to use, transfer design, peer support, supervisor sanction, personal outcomes-positive, personal 
outcomes-negative, change resistance, and content validity. The author's further literature research yielded seven new constructs that would fit the theoretical frame: selfefficacy, expectancy-related constructs, personal capacity for transfer, feedbackperformance coaching, learner readiness, and general motivation to transfer.

Holton and Bates (2000) then conducted exploratory factor analysis to evaluate the fit of the new hypothesized constructs. The average major factor loading was .62 with Cronbach alpha reliabilities ranging from .63 to .91 and only three of the scales below .70. Some of the items did not load or loaded weakly. The authors retained sixtyeight of the 112 items in the final instrument, assessing sixteen constructs. Through a second-order factor analysis, Holton et al. designated eleven of the sixteen constructs as training specific scales and five as general scales.

The LTSI instrument and others like it are important developments in the measurement of training transfer practices within organizations and serve as important platforms for further research. 


\title{
CHAPTER III \\ RESEARCH METHODOLOGY
}

\begin{abstract}
Overview
The transfer of training involves applying skills and knowledge acquired in a learning environment to the job environment. The study of what motivates trainees to take advantage of the training they experienced is critical to developing strategies to maximize the resources invested by the organization. Both situational and individual factors are components to the motivation of trainees to transfer training to the job. The model of transfer used in this study suggests that both situational and individual factors and the pre-training motivation to transfer which in turn, influences post-training motivation to transfer. This investigation tested the correlation between individual, situational, and pre-training motivation factors, and post-training motivation to transfer skills and learning. The research also examined the comparison between individual and situational factors and their affect on post-training motivation to transfer.

The participants in this course were employees of a large Midwestern-based insurance company who were attending classes to increase their job knowledge and skill in several operational areas. Participants had opportunity to learn and practice the skills in class and apply them to their jobs after leaving training. They were surveyed at the
\end{abstract}


beginning of training and after training. The two sets of data were then analyzed against the hypotheses of this study.

This chapter will present the study's context and research questions, introduce the overall study design, provide information concerning study participants and characteristics, discuss the instruments used in the study, and describe the specific procedures used to collect and analyze the data.

\section{$\underline{\text { Research Context }}$}

Call centers have become the customer communication method of choice for most companies with a public presence. For many companies like airlines, credit card companies, and insurance companies, call centers are the only link between the customer and the service provider (Gans, Koole \& Mandelbaum, 2003). Industry analysts report that agents working in U.S call centers reached 1.55 million in 1999 - more than 1.4\% of the private-sector employment - and was growing at a rate of $8 \%$ a year (Datamonitor, 2003).

This study took place in a large mid-western insurance company's call center training facility. For purposes of confidentiality, the company will be referred to with the fictitious name ABC Corporation. ABC Corp. call center employees in two Midwestern cities participated in this study. The general gender make-up of ABC Corporation call center employees is approximately $75 \%$ female and $25 \%$ male. Race and ethnic mix is comprised as follows: 80\% White American, 10\% African American, 3\% Asian American, and other ethnicities (Hispanic American and Native American) at less than 2\%. The average age of ABC Corp. call center associates is 34 with the range being 18 
to 63. The average tenure of $\mathrm{ABC}$ Corporation call center associates with the company is 4 years.

\section{$\underline{\text { Research Questions }}$}

Research hypotheses that represented the research questions are stated below followed by the statistical method used to test the hypothesis.

H1 Locus of control as measured by the eleven items in the pre-training survey will be positively correlated to motivation to transfer as measured by the six item post-training survey.

A Pearson correlation coefficient was used to determine the nature and magnitude of the relationship between learning confidence and motivation to transfer. Statistical significance was based on an alpha level of .05.

H2 General self efficacy as measured by the five items in the pre-training survey will be positively correlated to motivation to transfer as measured by the six item post-training survey.

A Pearson correlation coefficient was used to determine the nature and magnitude of the relationship between general self efficacy and motivation to transfer. Statistical significance was based on an alpha level of .05 . 
H3 Learning confidence as measured by the five items in the pre-training survey will be positively correlated to motivation to transfer as measured by the six item post-training survey.

A Pearson correlation coefficient was used to determine the nature and magnitude of the relationship between learning confidence and motivation to transfer. Statistical significance was based on an alpha level of .05.

H4 Motivation to learn as measured by the five items in the pretraining survey will be positively correlated to motivation to transfer as measured by the six item post-training survey.

A Pearson correlation coefficient was used to determine the nature and magnitude of the relationship between pre-training motivation to learn and motivation to transfer. Statistical significance was based on an alpha level of .05.

H5 Organizational support as measured by the five items in the pre-training survey will be positively correlated to motivation to transfer as measured by the six item post-training survey.

A Pearson correlation coefficient was used to determine the nature and magnitude of the relationship between organization support and motivation to transfer. Statistical significance was based on an alpha level of .05.

H6 Managerial support as measured by the five items in the pre-training survey will be positively correlated to motivation to transfer as measured by the six item post-training survey.

A Pearson correlation coefficient was used to determine the nature and magnitude of the relationship between manager support and motivation to transfer. Statistical significance was based on an alpha level of .05. 
H7 Job support as measured by the five items in the pre-training survey will be positively correlated to motivation to transfer as measured by the six item post-training survey.

A Pearson correlation coefficient was used to determine the nature and magnitude of the relationship between job support and motivation to transfer. Statistical significance was based on an alpha level of .05.

H8 Reaction to training as measured by the five items in the pretraining survey will be positively correlated to motivation to transfer as measured by the six item post-training survey.

A Pearson correlation coefficient was used to determine the nature and magnitude of the relationship between reaction to training and motivation to transfer. Statistical significance was based on an alpha level of .05.

H9 After controlling for demographic variables (age, race, and gender), individual characteristics variables (self-efficacy, motivation to learn, and learning confidence) will have a significant positive relationship with the criterion variable motivation to transfer training. In addition, after controlling for demographic variables and individual characteristics variables, situational variables (manager support, organizational support, and job support) will have a significant positive relationship with the criterion variable motivation to transfer training.

The three control variables of age, race, and gender were first entered into the equation. Then the three individual characteristic variables of self-efficacy, motivation to learn, and learning confidence) were entered into the equation. Finally the three situational characteristic variables of manager support, organization support, and job support were entered into the equation. Motivation to transfer was the criterion variable. Hierarchical multiple regression was used to determine the nature and magnitude of the 
relationship between the three sets of variables (demographic, individual characteristics, and situational characteristics) and motivation to transfer. Statistical significance was based on an alpha level of .05.

\section{$\underline{\text { Research Design }}$}

This research investigation is a longitudinal correlational study examining the relationships between predictor variables representing situational and individual factors, and trainees' motivation to transfer training within a call center environment. This study also explores the comparative influence of situational factors against individual factors on trainees' motivation to transfer training. The individual characteristic predictor variables were self-efficacy, motivation to learn, and learning confidence. The situational characteristic predictor variables were manager support, organizational support, and job support. Additionally, the individual predictor variables were combined and examined as one variable in relation to the combined situational predictor variables. The criterion variable was the trainees’ motivation to transfer training.

Trainee participants completed a questionnaire at the beginning of training comprised of six sub-scales measuring the predictor variables. Trainees then completed a second questionnaire at the conclusion of training comprised of a single scale measuring the reaction to training and the criterion variable of motivation to transfer training. 


\section{$\underline{\text { Participants }}$}

The data from this study were collected from customer service representatives of ABC Corporation, a large mid-western insurance company. The customer service representatives, or CSR position is an entry-level job within the organization. CSRs will typically have a high school or college education and little to some prior work experience. This population was selected because it is representative of thousands of other workers employed in the service sector in the U.S. The sample $(N=208)$ consisted of call center employees who were enrolled in one of three skill and knowledge courses relevant to their positions. The skill and knowledge courses included Commercial support, Claims, and Medicare courses. Each class was comprised of from 5 to 30 participants with an average attendance of 15 . The duration of each class ranged from 1 day to 5 days, with the average training class running for 2.5 days.

Two surveys were used in this study. Participants received the pre-training survey at the beginning of the training session and the follow-up survey at the completion of the training session. The overall response rate for the pre-training survey was $99 \%$, or 206 of the 208 surveyed. The return rate for the follow-up survey was $95 \%$, or 195 of the 206 who received the first survey. All students of each class were invited to participate in the survey.

In this study, the ratio of the number of subjects to the number of predictor variables is less than the suggested ratio of $15 / 1$ that is recommended for social science research data (Stevens, 2002). As a consequence, the $R$ squared value for the regression equation from the sample will be inflated, as compared to the $R$ squared value in the 
population. In order to provide an accurate estimate of the population $R$ squared, a conservative adjustment will be calculated from the sample data. The Stein adjustment formula for adjusted $R$ square (Stevens, 2002) will be calculated. The Stein adjusted $R$ squared is the average proportion of variance in the dependent variable accounted for by the predictor variables if the researcher were to apply the regression equation to many other samples from the population.

The average age of the participants who completed both surveys was 34. Female and male composition of the participants was $74 \%$ and $25 \%$ respectively. All of the participants were full-time employees of ABC Corp.. The demographic mix of the participants was 82\% White American, 10\% African American, 2\% Hispanic American, 4\% Asian American, 1\% Native American, and 2\% Other.

Of the 195 surveys completed during the training courses, 124 or $63 \%$ provided data sufficient for analysis. Those missing more than $20 \%$ of the data were not used in the analysis and removed from the data set. The missing data was primarily due to one variable, tenure with the organization. Because this was not a primary inferential variable of interest in the study, the average tenure (48 months) was used in cases where tenure data was missing. Once the average tenure was added to cases to replace the missing data, 185 surveys, or 94\% provided data sufficient for analysis.

\section{$\underline{\text { Instruments Used in Data Collection }}$}

Two survey instruments were used in this research. Participants first completed a 53-item survey at the beginning of their training. The survey was comprised of items 
from scales representing the six predictor variables in the study: managerial support, job support, organizational support, self efficacy, motivation to learn, and learning confidence. All items were scored on a five-point Likert type scale $(5=$ Strongly Agree to 1 = Strongly Disagree). The survey asked participants to consider the items as they applied to themselves for the first 38 items representing individual characteristics. The survey then asked participants to consider the items as they applied to their organization for the remaining 15 items representing situational characteristics.

Participants completed a second survey instrument after the completion of training but before leaving class. The second survey was comprised of 17 items from a scale representing the criterion variable of motivation to transfer. All items on this survey were scored in a similar manner to the first survey (Likert 5-point scale with 5 = Strongly Agree to 1 = Strongly Disagree) .

\section{Linking the Surveys and Response Rate}

Because this was a longitudinal study, linking each of the participant's two surveys was necessary. To avoid the use of personal or confidential identification codes such as social security or employee numbers, this study utilized a self-generated identification code for anonymous collection of longitudinal questionnaire data developed by Kearney, Hopkins, Armand and Weisheit (1984).

Using this technique, participants create a code comprised of code elements that are well known to them, but not the researcher. Examples include middle initials, birth months, or street numbers. The elements chosen must remain constant over the duration 
of the study so that participants can easily recall the individual elements to re-create the code. While there is a possibility that exact matches may not occur for all participants, and therefore lower the matching rate, allowing for one of the elements to be off in the pair still supplies enough information to be reasonably sure that the surveys are from the same person. Researchers have shown this method yields $92 \%$ reliability over a one month time interval and 78\% over a one year interval (Kearney et. al 1984).

In this study, participants created a seven-element code based on the following code elements: first letter of middle name, first letter of mother's first name, gender (M or F), birth month number, first letter of birth state or country (if not U.S. born), number of older brothers and sisters in family, and first number of street address. Participants wrote this self-generated code on both surveys in a box provided for this purpose.

Of the 208 employees attending training who were invited to participate in the survey, 206 chose to participate. Of the 206 pre-training surveys collected, 195 or $94.6 \%$ exactly matched a corresponding second survey. There were 11 pre-training surveys or 5.4\% that either did not match, or there was no corresponding post-training surveys returned. Two potential participants declined to participate in either survey. The total participation rate, including non-responders on one or both surveys, was $93.8 \%$.

\section{Predictor Variables}

Predictor variables used in this study include perceptions of managerial support, job support, organizational support, self-efficacy, motivation to learn, learning 
confidence, gender, age, and race. Gender, age, and race were self reported by participants on the survey form.

Managerial support. Managerial support was measured using a five item scale developed by Tracey, Tannenbaum, and Kavanagh (1995). Sample questions included: “Supervisors match associates’ needs for personal and professional development with opportunities to attend training," and "Supervisors give recognition and credit to those who apply new knowledge and skills to their work.” The coefficient alpha for the managerial support scale was .87 (Tracey \& Tews, 2003).

Job support. Job support was measured using a five item scale developed by Tracey, Tannenbaum, and Kavanagh (1995). Sample questions included: "Gaining new information about ways to perform work more effectively is important in this organization,” and "Work assignments include opportunities to learn new techniques and procedures for improving performance.” The coefficient alpha for the job support scale was .85 (Tracey \& Tews, 2003).

Organization support. Organization support was measured using a five item scale developed by Tracey, Tannenbaum, and Kavanagh (1995). Sample questions included: "There is a performance appraisal system that ties financial rewards to use of newly acquired knowledge and skills," and "Employees are provided with resources necessary to acquire and use new knowledge and skills.” The coefficient alpha for the organization support scale was .87 (Tracey \& Tews, 2003).

Learning Confidence. Learning confidence was measured using a five item scale developed by Warr, Allan, and Birdi (1999). Sample questions included: “I don’t have 
many problems picking up new skills," and "I am quite nervous about coming on this course (reverse scored).” The coefficient alpha for the learning confidence scale was .80 . General Self Efficacy. General Self Efficacy was measured using a 17-item scale developed by Sherer, Maddux, Mercadente, Prentice-Dunn, and Rogers (1982). Sample questions included: "If something looks too complicated, I will not even bother to try it," and "I do not seem capable of dealing with most problems that come up in my life."

Motivation to Learn. Motivation to learn was measured using a six item scale developed by Noe (1986). Sample questions included: "I am motivated to learn the skills emphasized in the training program," and "I will get more from this training program than most people" The coefficient alpha for the motivation to learn scale was .88 .

Trainee Reactions. Trainee reaction to training was measured using a nine item scale with three subscales developed by Warr, Allan, and Birdi (1999). The subscale Enjoyment included questions such as "I really enjoyed this course", and "This course was extremely interesting." The coefficient alpha for this subscale was .76. The subscale Perceived Usefulness included questions such as "This course was of great practical value to me for my job," and "This course was closely related to my job needs.” The coefficient alpha for this subscale was .80. The subscale Perceived Difficulty included questions such as "I found this course very hard to follow," and "I found this course difficult to understand.” The coefficient alpha for this subscale was .79.

\section{Criterion Variable}

The criterion variable for this study was motivation to transfer skills to the job. Motivation to transfer skills to the job was measured using a six-item scale developed by 
Noe and Schmitt (1986). Sample questions included: "I feel capable of using the skills developed in this course in my everyday work," and "I know of work situations in which I plan to use what I have learned in this course.” The alpha coefficient for this scale was .95 .

\section{Procedure}

Participants in each of the training programs received pre-training instructions from a survey administrator who was trained to implement the surveys in a consistent manner. The administrator welcomed the participants and explained the purpose of the survey was to investigate how people are motivated to use training on the job and that those willing to participate would complete two surveys, one at the beginning of training and one at the conclusion of training. The administrator then read a script that explained the letter of consent and informed participants that participation was voluntary and that they could opt out of the process at any time. Participants then received the Informed Letter of Consent to review and sign, and the administrator then collected the participant's signed forms (See Appendix A). Participants were allowed to retain a copy of the Informed Letter of Consent form for their personal records.

The administrator then distributed the first survey to the participants and explained the necessity of using the 7-item self-generated identification code to link the surveys together. The informed consent letter also explained the necessity of using a self-generated code and the administrator underscored this point. The administrator then told the participants that the code was for data collection only and that once the data from 
the second survey had been collected and analyzed, the codes would be destroyed. The administrator also stressed that no-one other than the administrator and the principle researchers would handle the surveys and codes. Participants created their codes and wrote them in the designated area on the survey form.

The participants completed the 53-item pre-training survey. The pre-training survey was comprised of six sub-scales which measured learning confidence (5 items), self efficacy (17 items), manager support (5 items), job support (5 items), organization support (5 items), and motivation to learn (6 items). After the training was completed, the participants completed the 17-item post-training survey. The second survey was comprised of two sub-scales which measured participant reaction (9 items) and motivation to transfer skills ( 8 items.). The administrator collected the surveys and the researcher matched the self-generated identification codes from the two surveys to form longitudinal pairs.

\section{Data Analysis}

Descriptive statistics were used to describe the demographic data including, gender, age, race/ethnicity, years with the company, and education level. Since the primary statistical test in this research was multiple regression analysis, appropriate tests were conducted to satisfy the four major assumptions of multiple regression: normality, linearity, reliability, and homoscedasticity. Bivariate correlations were used to check for multicollinearity and describe the relationships between learning confidence, self- 
efficacy, manager support, job support, organizational support, pre-training motivation, and reaction to training. Scatter plots and histograms were generated to confirm assumptions of normality and homoscedasticity and it was found that the distribution was normal and there were an equal number of cases above and below the regression line. Hierarchical multiple regression analysis was used for all research questions to determine the predictive relationships between individual/situational factors and post-training motivation. The control variables of age, race, and gender were entered first in each of the regression analyses, followed by the predictor variable considered in each test. Because Cronbach's alphas for all predictor variable scales exceeded .70, they were considered appropriate for use in multiple regression analysis. 


\section{CHAPTER IV}

\section{RESULTS}

\section{$\underline{\text { Introduction }}$}

The research studied the predictive relationships between situational characteristics, individual characteristics, reaction to training, and the criterion variable post-training motivation to transfer training. The participants in this study were customer service representatives employed by $\mathrm{ABC}$ Corporation, a large insurance company located in the Midwest. The questionnaires used in the study measured: (a) demographics of ABC Corp. call center employees, (b) situational characteristics, (c) individual characteristics, (d) reaction to training, and (e) post-training motivation to transfer training. The study was conducted in a longitudinal manner. Participants completed a pre-training survey measuring individual and situational characteristics, followed by a post-training survey measuring reaction to training and motivation to transfer. Individual characteristic items were adapted from research by Noe (1986), Sherer, Maddux, Mercadente, Prentice-Dunn, and Rogers (1982), and Warr, Allan, and Birdi (1999). Situational characteristic items were adapted from research by Tracey, Tannenbaum, and Kavanagh (1995). The measurement for the criterion variable of motivation to transfer was adapted from research by Noe (1986). 
This chapter presents the study results obtained through quantitative analysis of the questionnaire data. The independent or predictor variables were: (a) general selfefficacy, (b) motivation to learn, (c) learning confidence, (d) locus of control, (e) organizational support, (f) managerial support, (g) job support, and (h) reaction to training. The dependent or criterion variable was motivation to transfer. The two main statistical procedures were Pearson correlation and hierarchical regression analysis. Though unanticipated in the study design, factor analysis was also used in the analysis to further clarify and consolidate predictor variables used in the regression analysis. Quantitative analysis was performed using the Statistical Package for Social Sciences (SPSS).

The four questions that guided this study were:

1. What is the relationship between individual factors (self efficacy, learning confidence, motivation to learn) and motivation to transfer training in a callcenter environment?

2. What is the relationship between situational factors (manager support, job support, and organizational support) and motivation to transfer training in a call-center environment?

3. What is the relationship between post-training reaction and motivation to transfer training in a call center environment?

4. Controlling for demographic variables, what is the predictive relationship between individual variables and situational variables and motivation to transfer in a call center environment? 
Results are presented in this chapter for these questions and include descriptive statistics, reliability statistics for each scale, and descriptions of data analysis for research questions one, two, three and four. Nine hypotheses were used to address these questions and results for each will be discussed.

\section{Summary of Characteristics of the Sample}

During the study, 208 participants received the pre-training survey. Out of this number, 206 completed the first survey. The resulting response rate on the first survey was 99\%. Of the 206 participants who completed the first survey, 195 completed the second post-training survey. The resulting response rate on the second survey was 95\%. The overall response rate for both surveys combined was 94\%. Table 1 shows the number of respondents by survey.

\section{Table 1}

Study Response by Survey

\begin{tabular}{lccc}
\hline Survey & $\begin{array}{l}\text { Number of Training } \\
\text { Participants }\end{array}$ & $\begin{array}{l}\text { Number of } \\
\text { Responses }\end{array}$ & $\begin{array}{l}\text { \% of } \\
\text { Responses }\end{array}$ \\
\hline Pre-Training Survey & 208 & 206 & 99 \\
Post-Training Survey & 206 & 195 & 95 \\
Combined Surveys & 208 & 195 & 94 \\
\hline
\end{tabular}


Demographic variables measured on the training participants included gender, ethnicity, age, and marital status. The survey also obtained information on tenure with ABC Corp. and education level. Table 2 summarized the demographic characteristics by gender. Most of the participants were women. Of the 195 participants, 145 (74\%) were female and 48 (25\%) were male.

Table 2

Distribution of Respondents by Gender

\begin{tabular}{lcc}
\hline Gender & $n$ & $\%$ \\
\hline Female & 145 & 74 \\
Male & 48 & 25 \\
Missing & 2 & 1 \\
\hline
\end{tabular}

Ethnicity characteristics in the sample are summarized in Table 3. Of the 195 participants, 160 (82\%) were White American, 20 (10\%) were African American, seven ( 4\%) were Asian American, three (2\%) were Hispanic American, one (1\%) was Native American, and three (2\%) classified themselves as something other than the stated categories. 
Table 3

Distribution of Respondents by Ethnicity (Using Six Categories)

\begin{tabular}{lcc}
\hline Ethnicity & $n$ & $\%$ \\
\hline African American & 20 & 10 \\
White American & 160 & 82 \\
Hispanic American & 3 & 2 \\
Asian American & 7 & 4 \\
Native American & 1 & 1 \\
Other & 3 & 2 \\
\hline
\end{tabular}

Table 4 summarizes the characteristics of the sample group of participants by age group. Participants were most represented in the 25 to 34 age bracket (43\%) followed by the 35 to 44 age bracket (27\%).

Table 4

Distribution of Respondents by Age (Using Six Categories)

\begin{tabular}{lcc}
\hline Age & $n$ & $\%$ \\
\hline 55 and Above & 9 & 5 \\
$45-54$ & 22 & 11 \\
$35-44$ & 53 & 27 \\
$25-34$ & 83 & 27 \\
Below 25 & 28 & 14 \\
\hline
\end{tabular}


Table 5 represents the distribution of participants by level of education. All participants were at least high school graduates. Four-year college degrees represented the highest level of education for the most participants (41\%) followed by high school graduates $(37 \%)$.

Table 5

Distribution of Respondents by Education Level (Using Six Categories)

\begin{tabular}{lll}
\hline Education Level & $n$ & $\%$
\end{tabular}

Doctoral or professional degree 0

0

Master's degree 10 5

Four year college graduate 79 41

Two year college graduate 33 17

High school graduate 71 37

Did not graduate from high school

0 0

Table 6 represents the distribution of participants by years of job experience with ABC Corp. Most of the participants had from 0 to 2 years experience (64\%) followed by those with 2 to 6 years experience (23\%). 
Table 6

Distribution of Respondents by Years of Experience at ABC Corporation

\begin{tabular}{lcc}
\hline Years of Experience & $n$ & $\%$ \\
\hline$>10$ & 17 & 11 \\
$7-10$ & 13 & 9 \\
$2-6$ & 23 & 15 \\
$<2$ & 96 & 64 \\
\hline
\end{tabular}

Summary of Reliability Coefficients for Each Scale

Internal consistency reliability coefficients were calculated for the seven sections of the motivation to transfer pre-training questionnaire: General Self-efficacy, Learning Confidence, Motivation to Learn, Locus of Control, Organizational Support, Managerial Support, and Job Support. Reliability coefficients were also calculated for the two sections of the post-training questionnaire: Reaction to Training, and Motivation to Transfer. Coefficient Alpha is the commonly accepted statistical test in empirical social science research to determine the reliability of parallel items. As Table 7 illustrates, with the exception of Locus of Control, reliabilities for all scales were above the minimum level considered acceptable for research (Nunnaly \& Bernstein, 1994). 
Table 7

Reliability Statistics for Nine Scales

\begin{tabular}{lll}
\hline Scale & $\begin{array}{l}\text { Cronbach’s } \\
\text { Alpha }\end{array}$ & $\begin{array}{l}\text { N of } \\
\text { Items }\end{array}$ \\
\hline General Self Efficacy & .85 & 16 \\
Learning Confidence & .75 & 5 \\
Motivation to Learn & .74 & 6 \\
Locus of Control & .55 & 11 \\
Organizational Support & .86 & 5 \\
Managerial Support & .75 & 5 \\
Job Support & .88 & 5 \\
Reaction to Training & .83 & 11 \\
Motivation to Transfer & .83 & 6 \\
\hline
\end{tabular}

\section{$\underline{\text { Relationships among Predictor Variables }}$}

The study analyzed comparisons between predictor variables of interest including five demographic variables and nine variables representing situational factors, individual factors, and reaction to training. The means, standard deviations, and intercorrelations for the predictor variables and the criterion variable of Motivation to Transfer Training are presented in Table 8. 


\section{Table 8}

Means, Standard Deviations and Intercorrelations for Motivation to Transfer and Predictor Variables $(n=185)$

\begin{tabular}{|c|c|c|c|c|c|c|c|c|c|c|c|c|c|c|c|}
\hline Variable & $\mathbf{M}$ & SD & 1 & 2 & 3 & 4 & 5 & 6 & 7 & 8 & 9 & 10 & 11 & 12 & 13 \\
\hline Motivation to Transfer & 4.14 & 0.55 & -.02 & -.01 & .01 & $-.18 * *$ & -.03 & .11 & $.18^{* *}$ & $.26 * * .3$ & $.36^{* *} .3$ & $.39 * *$ & $.40^{* *}$ & $.40^{* *}$ & $.72^{* *}$ \\
\hline \multicolumn{16}{|l|}{ Predictor Variable } \\
\hline 1. Age & 34.5 & 9.70 & -- & $.18^{* *}$ & $-.29 * *$ & $.29 * *$ & $-.22 * *$ & .07 & .01 & $-.18^{* *}$ & .08 & -.09 & $-.15^{*}$ & -.11 & -.10 \\
\hline 2. Gender & 1.75 & 0.44 & & -- & -.01 & $.18^{* *}$ & $-.31 * *$ & .03 & $.15^{*}$ & -.17 & $.21^{* *}$ & -.11 & -.07 & -.06 & -.06 \\
\hline 3. Marital Status & 1.50 & 0.50 & & & -- & $-.14 *$ & .09 & -.01 & .07 & .04 & .05 & .05 & .09 & .06 & .07 \\
\hline 4. Tenure with ABC Corp. & 41.70 & 48.30 & & & & -- & $-.26 * *$ & .01 & $-.14 *$ & $-.15^{*}$ & -.10 & $-.25^{* *}$ & $-.30 * *$ & $-.19 * *$ & $-.19 * *$ \\
\hline 5. Education Level & 3.16 & 0.99 & & & & & -- & -.07 & -.04 & .12 & $-.12 *-$ & -.07 & .05 & -.01 & -.10 \\
\hline 6. Locus of Control & 3.63 & 0.43 & & & & & & -- & $.34 * *$ & $.23 * *$ & $.33 * *$ & $.20 * *$ & $.22 * *$ & $.31 * *$ & $.14^{*}$ \\
\hline 7. General Self-Efficacy & 4.06 & 0.37 & & & & & & & -- & $.53^{* *}$ & $.46^{* *}$ & .10 & $.19 * *$ & $.16^{*}$ & $.21^{* *}$ \\
\hline 8. Learning Confidence & 4.17 & 0.51 & & & & & & & & -- & $.29 * *$ & .12 & $.23^{* *}$ & $.20 * *$ & $.29 * *$ \\
\hline 9. Motivation to Learn & 3.94 & 0.51 & & & & & & & & & -- & $.36^{* *}$ & $.35^{* *}$ & $.45^{* *}$ & $.25^{* *}$ \\
\hline 10. Organizational Support & 3.30 & 0.77 & & & & & & & & & & -- & $.76^{* *}$ & $.79 * *$ & $.34^{* *}$ \\
\hline 11. Managerial Support & 3.64 & 0.60 & & & & & & & & & & & -- & $.79 * *$ & $.38 * *$ \\
\hline 12. Job Support & 3.73 & 0.68 & & & & & & & & & & & & -- & $.38 * *$ \\
\hline 13. Reaction to Training & 4.07 & 0.45 & & & & & & & & & & & & & -- \\
\hline
\end{tabular}

$* p<.05, \quad * * p<.01$ 


\section{$\underline{\text { Demographic Correlations }}$}

In general, the demographic variables of age, gender, marital status, and education level were weakly correlated with the other variables of interest. The one notable exception, tenure with the company, was negatively correlated with almost all individual and situational variables. The negative correlation for tenure was the moderate with organizational support $(r=-.25, p<.01)$ and managerial support $(r=-.30, p<.01)$. These findings suggest that the more tenured employees were somewhat likely to be less positive about the support they received from both their managers and the organization as a whole.

\section{$\underline{\text { Individual Variable Correlations }}$}

The individual factors of locus of control, general self-efficacy, learning confidence, and motivation to learn were positively and significantly correlated with the other variables of interest. General self-efficacy, in particular, had moderately strong correlations with learning confidence $(r=.53, p<.01)$ and motivation to learn $(r=.46$, $p<.01)$. Locus of control also had moderate correlations with general self-efficacy $(r=$ $.34, p<.01)$, motivation to learn $(r=.33, p<.01)$, and job support $(r=.31, p<.01)$. These findings suggest that there may be moderate to moderately strong overlap in how a one perceives his/her concept of self control and determination and how one perceives his/her ability to learn. 


\section{$\underline{\text { Situational Variable Correlations }}$}

The situational variables of organizational support, managerial support, and job support were significantly and strongly correlated with each other. Organizational support was strongly correlated with managerial support $(r=.76, p<.01)$ and job support $(r=.79, p<.01)$. Managerial support and job support were also strongly correlated $(r=$ $.79, p<.01)$. The strong and significant correlations among these situational variables suggest that subjects may not draw clear lines of distinction between manager, job, and company.

\section{$\underline{\text { Reaction to Training Correlations }}$}

Reaction to training was most moderately and significantly correlated with all variables of interest. It was most strongly correlated with the situational variables organizational support $(r=.34, p<.01)$, managerial support $(r=.38, p<.01)$, and job support $(r=.38, p<.01)$. The participants who felt the most positive about training also felt that their job, manager, and organization were the most supportive.

\section{Research Question One}

Research question one examined the relationship between individual characteristic factors (locus of control, self efficacy, learning confidence, motivation to learn) and motivation to transfer training in a call-center environment. This question encompassed the first four hypotheses. For these hypotheses, Pearson correlations were 
used to evaluate the strength and significance of the relationships. The results of these tests for each hypothesis are presented below.

H1 Locus of control as measured by the eleven items in the pre-training survey will be positively correlated to motivation to transfer as measured by the six item post-training survey.

The correlation coefficient for the relationship between locus of control and the criterion variable motivation to transfer training was not significant at an alpha level of .05. How training participants view control of life events appears to have no correlation to how motivated they are to transfer training. The research hypothesis is not supported.

H2 General self efficacy as measured by the five items in the pre-training survey will be positively correlated to motivation to transfer as measured by the six item post-training survey.

The correlation coefficient for the relationship between general self-efficacy and the criterion variable motivation to transfer training was relatively weak, but statistically significant $(r=.18, p<.01)$. These findings support the hypothesis and suggest that internal factors that measure how one views belief in self have a small relation with motivation to transfer training.

H3 Learning confidence as measured by the five items in the pre-training survey will be positively correlated to motivation to transfer as measured by the six item post-training survey.

The correlation coefficient for the relationship between learning confidence and the criterion variable motivation to transfer training was moderately weak and statistically significant $(r=.26, p<.01)$. These findings support the hypothesis and suggest that training participants' confidence in learning new material is somewhat correlated to how motivated they are to transfer training to the job. 
H4 Motivation to learn as measured by the five items in the pretraining survey will be positively correlated to motivation to transfer as measured by the six item post-training survey.

The correlation coefficient for the relationship between motivation to learn and the criterion variable motivation to transfer training was moderate and statistically significant $(r=.36, p<.01)$. These findings support the research hypothesis and suggest that training participants’ motivation to learn has a moderately positive relationship with how motivated they are to transfer training to the job.

\section{Research Question Two}

Research question two examined the relationship between situational characteristic factors (manager support, job support, and organizational support) and motivation to transfer training in a call-center environment. This question encompassed hypotheses five through seven. For these hypotheses, Pearson correlations were used to evaluate the strength and significance of the relationships. The results of these tests for each hypothesis are presented below.

H5 Organizational support as measured by the five items in the pre-training survey will be positively correlated to motivation to transfer as measured by the six item post-training survey.

The correlation coefficient for the relationship between organizational support and the criterion variable motivation to transfer training was moderate and statistically significant $(r=.39, p<.01)$. These findings support the research hypothesis and suggest that the support participants perceive receiving from the larger organization has a 
moderately positive relationship with how motivated they are to transfer training to the job.

H6 Managerial support as measured by the five items in the pre-training survey will be positively correlated to motivation to transfer as measured by the six item post-training survey.

The correlation coefficient for the relationship between managerial support and the criterion variable motivation to transfer training was moderate and statistically significant $(r=.40, p<.01)$. These findings support the research hypothesis and suggest that the support participants perceive receiving from their manager has a moderately direct relationship with how motivated they are to transfer training to the job. Because both organizational support and managerial support variables were strongly correlated to each other $(r=.76, p<.01)$, we can infer that these variables are viewed by participants as measuring a similar underlying variable.

$\mathrm{H} 7 \quad$ Job support as measured by the five items in the pre-training survey will be positively correlated to motivation to transfer as measured by the six item post-training survey.

The correlation coefficient for the relationship between job support and the criterion variable motivation to transfer training was moderate and statistically significant $(r=.40, p<.01)$. These findings support the research hypothesis and suggest that participants who positively perceive the design of their job are also more likely to be motivated to transfer training to the job. Job support was also closely correlated to managerial support and organizational support. It is a reasonable inference that these 
three situational variables measure much the same thing in the eyes of the training participant.

\section{Research Question Three}

Research question three examined the relationship between post-training reaction and motivation to transfer training in a call center environment. This question encompassed hypothesis eight. For this hypothesis, Pearson correlation was used to evaluate the strength and statistical significance of the relationships. The results of this test for the hypothesis are presented below:

H8 Reaction to training as measured by the five items in the pretraining survey will be positively correlated to motivation to transfer as measured by the six item post-training survey.

The correlation coefficient for the relationship between reaction to training and the criterion variable motivation to transfer training was strong and statistically significant $(r=.72, p<.01)$.

\section{Research Question Four}

Research question four examined the predictive relationship between individual and situational variables and motivation to transfer in a call center environment after controlling for demographic variables. For this hypothesis, hierarchical multiple regression and factor analysis were used to evaluate the strength and significance of the relationships. The results of this test for the hypothesis are presented below: 
H9 After controlling for demographic variables (age, gender, marital status, education, and tenure), individual characteristics variables (locus of control, self-efficacy, motivation to learn, and learning confidence) will have a significant positive relationship with the criterion variable motivation to transfer training. In addition, after controlling for demographic variables and individual characteristics variables, situational variables (managerial support, organizational support, and job support) will have a significant positive relationship with the criterion variable motivation to transfer training.

\section{$\underline{\text { First Regression Analysis }}$}

Hierarchical regression was used to test this hypothesis by regressing the motivation to transfer training scale against the demographic variables and the eight predictor variables: locus of control, self-efficacy, motivation to learn, learning confidence, managerial support, organizational support, job support, and reaction to training. Because of missing data for the tenure variable, the initial regression analysis yielded only 128 useable cases. With nine predictor variables and a minimum of 15 cases needed per predictor variable (Stevens, 1994), it was determined that an average of the existing data set for tenure would be used for the missing data. This practice is common in social science research where missing data prohibits the sample from being large enough to satisfy statistical test requirements.

The regression analysis was a constructed in four models according and based on the model presented by Mathieu and Martineau (1997). The demographic variables (age, gender, marital status, tenure, and education) were entered in the first model and regressed against motivation to transfer training. The second model included the demographic variables and added the individual factor variables: locus of control, general self-efficacy, learning confidence, and motivation to learn. The third model included the 
previous variables with the addition of the situational factor variables: organizational support, managerial support, and job support. Finally, the fourth model included all previous variables with the addition of the reaction to training variable.

The outcome of this analysis was a multiple correlation coefficient $\left(R^{2}\right)$ that represented the degree of relationship between the nine independent variables and the dependent variable. Examination of the plots of the data and residual statistics showed that the statistical assumptions of normality, linearity, and homoscedasticity were not violated. However, there was evidence of multicollinearity and this problem was addressed in a second regression analysis.

With just the demographic variables of age, gender, marital status, tenure, and education in the first model, $4 \%$ of the variance in the dependent variable was predicted. By adding the individual factor variables of locus of control, learning confidence, motivation to learn, and general self-efficacy, the variance explained increased to $18 \%$ in the second model. By then adding the situational factor variables of managerial support, organization support, and job support, the explained variance increased to $26 \%$ in the third model. Finally, when the reaction to training predictor variable was added to the equation, the explained variance increased to $58 \%$ in the fourth model. Table 9 presents the $R^{2}$, change in $R^{2}$ and the standard error of the estimate for each step in the equation. The standard error of the estimate is the standard deviation of predictions score errors from the regression line. The lower the error number the higher the degree of a linear relationship in the regression equation (Vogt, 1999). The standard error of estimate for all four models is relatively low, suggesting a higher level of confidence in the model's linear relationships. 
Table 9

$R$ Squared Statistics and Standard Error of Estimate for Four Regression Models with Demographic Variables, Individual Variables, Situational Variables and Reaction to Training Predicting Motivation to Transfer Training

\begin{tabular}{ccccc}
\hline Model & $R$ & $R$ square & Adjusted $R$ Square & Std. Error of the Estimate \\
\hline 1 & $.202^{\mathrm{a}}$ & .041 & .014 & 0.54 \\
2 & $.429^{\mathrm{b}}$ & .184 & .142 & 0.51 \\
3 & $.508^{\mathrm{c}}$ & .258 & .206 & 0.49 \\
4 & $.761^{\mathrm{d}}$ & .580 & .548 & 0.37 \\
\hline
\end{tabular}

a Predictors: Constant, Education, Marital, Tenure, Gender, Age

b Predictors: Constant, Education, Marital, Tenure, Gender, Age, Locus of Control, Learning Confidence, Motivation to Learn, General Self-Efficacy

c Predictors: Constant, Education, Marital, Tenure, Gender, Age, Locus of Control, Learning Confidence, Motivation to Learn, General Self-Efficacy, Managerial Support, Organizational Support, Job Support

d Predictors: Constant, Education, Marital, Tenure, Gender, Age, Locus of Control, Learning Confidence, Motivation to Learn, General Self-Efficacy, Managerial Support, Organizational Support, Job Support, Reaction to Training

Note. Dependent variable: Motivation to Transfer Training

Table 10 presents the specific variables listed in each model, the multiple correlation coefficient $\left(R^{2}\right)$, change in $R^{2}$, and the beta coefficients for each variable in each model. The overall $R^{2}$ for the final model was .580, but applying the Stein formula reduced the variance predicted by the fourth model to .510. The Stein formula is used as a more conservative measure in order to apply predictability to the overall population represented by the sample (Stevens, 2002). The beta coefficient portrays the difference in a dependent variable associated with an increase (or decrease) of one standard deviation in an independent variable, when controlling for the effects of the other independent variables (Vogt, 1999). 


\section{Table 10}

Hierarchical Regression Model Summary: Motivation to Transfer Training Predicted by Four Categories of Variables

\begin{tabular}{|c|c|c|c|}
\hline $\begin{array}{l}\text { Step and Predictor Variable } \\
\text {. }\end{array}$ & $\mathrm{R}^{2}$ & $\triangle \mathrm{R}^{2}$ & $\beta$ \\
\hline$\overline{\text { Model } 1}$ & .04 & .04 & \\
\hline Age & & & .03 \\
\hline Gender & & & .07 \\
\hline Marital & & & .00 \\
\hline Tenure & & & $-.21 * *$ \\
\hline Education & & & -.08 \\
\hline Model 2 & .18 & .14 & \\
\hline Age & & & 03 \\
\hline Gender & & & -.04 \\
\hline Marital & & & -.01 \\
\hline Tenure & & & $-1.5^{*}$ \\
\hline Education & & & -.07 \\
\hline Locus of Control & & & -.01 \\
\hline General Self-Efficacy & & & .10 \\
\hline Learning Confidence & & & .21 \\
\hline Motivation to Learn & & & $.33^{* *}$ \\
\hline Model 3 & .26 & .12 & \\
\hline Age & & & .06 \\
\hline Gender & & & .00 \\
\hline Marital & & & -.02 \\
\hline Tenure & & & -.08 \\
\hline Education & & & -.04 \\
\hline Locus of Control & & & -.06 \\
\hline General Self-Efficacy & & & -.06 \\
\hline Learning Confidence & & & $.19 *$ \\
\hline Motivation to Learn & & & $.20 *$ \\
\hline Organizational Support & & & .13 \\
\hline Managerial Support & & & .12 \\
\hline Job Support & & & .10 \\
\hline Model 4 & .58 & .26 & \\
\hline Age & & & .07 \\
\hline Gender & & & .04 \\
\hline Marital & & & -.04 \\
\hline Tenure & & & -.02 \\
\hline Education & & & .08 \\
\hline Locus of Control & & & -.05 \\
\hline General Self-Efficacy & & & -.08 \\
\hline Learning Confidence & & & .07 \\
\hline Motivation to Learn & & & $.18^{* *}$ \\
\hline Organizational Support & & & .13 \\
\hline Managerial Support & & & .02 \\
\hline Job Support & & & -.02 \\
\hline Reaction to Training & & & $.65^{* *}$ \\
\hline
\end{tabular}

${ }^{*} \mathrm{p}<.05 \quad{ }^{* *} \mathrm{p}<.01$ 
As presented in Table 10, reaction to training was by far the strongest predictor variable in the fourth model $(\beta=.65, p<.01)$ followed by motivation to learn $(\beta=.33, p$ $<.01)$ in the second model. Controlling for all other variables we conclude that the higher the reaction to training, the higher the motivation to transfer training. In the fourth model, situational factor variables considered individually did not predict for motivation to transfer training after reaction to training was entered in the equation. The only predictor variable to achieve positive predictive significance was motivation to learn ( $\beta=$ $.18, p<.01)$, but it was a relatively weak relationship.

\section{$\underline{\text { Second Regression Analysis with Reduced Number of Predictor Variables }}$}

As stated earlier, the strong multicollinearity expressed by the situational factor predictor variables and moderate multicollinearity expressed by the individual factor predictor variables prompted further examination and testing. Multicollinearity is the extent to which independent variables are correlated with one another. Its presence is problematic in regression analysis because it makes it very difficult to examine the separate effects of the independent variables on the dependent variable (Vogt, 1999). One remedy for dealing with multicollinearity where independent variables have intercorrelations of .80 or larger, is to perform a principal components, or factor analysis to reduce the predictor variables into a smaller set of predictors (Stevens, 2002). While the intercorrelations in this study were not .80 , they were sufficiently high to justify the possibility of using factor analysis.

In order to reduce the effects of multicollinearity, a factor analysis was performed on both the individual variable set and the situational variable set. Principal component 
analysis was the method used in extracting the factors. As presented in Table 11, all items for the individual characteristic variable set combined to form one factor with an initial eigenvalue of 2.1. An eigenvalue is a factor analysis statistic used to indicate how much of the variation in the original group of variables is accounted for by a particular factor. An eigenvalue of greater than one is considered indicative of a separate component (Vogt, 1999; Stevens, 2002).

Once the individual characteristic and situational characteristic scales were reduced to two factors, hierarchical regression was used to test this hypothesis by regressing the motivation to transfer training scale against the demographic variables and three additional predictor variables: individual factors, situational factors, and reaction to training. Because of missing data for the tenure variable, it was determined that an average of the existing data set for tenure would be used for the missing data.

Table 11

Factor Component Matrix for Individual Characteristic Variables

Scale Component 1

Locus of Control $\quad .636$

General Self-Efficacy $\quad .832$

Learning Confidence $\quad .711$

Motivation to Learn $\quad .702$

Table 12 shows all items for the situational characteristic variable set combined to form one factor with an initial eigenvalue of 2.6. 
Table 12

Factor Component Matrix for Situational Characteristic Variables

Scale Component 1

Organization Support $\quad .924$

Managerial Support $\quad .922$

Job Support $\quad .926$

Orthagonal varimax rotation was not used for either test because all variables loaded on a single factor. Bartlett’s Test of Sphericity and the Kaiser-Meyer-Olkin Measure of Sampling Adequacy both yielded acceptable scores for both factor analyses. The Bartlett Test for both analyses was statistically significant at the $p<.00$. Sampling adequacy measured by the Kaiser-Meyer-Olkin statistic was .685 and .760 for the individual characteristic and situational characteristic variables respectively. Scores above .5 indicate the factor analysis is acceptable.

The new variables, individual characteristics and situational characteristics were used in another hierarchical regression analysis after the demographic variables, but before the training reaction variable. Table 13 presents the $R^{2}$, change in $R^{2}$ and the standard error of the estimate for each step in the equation. 
Table 13

$R$ Squared Statistics and Standard Error of Estimate for

Four Regression Models using Individual Factors and Situational Factors

\begin{tabular}{ccccc}
\hline Model & $R$ & $R$ square & Adjusted $R$ Square & $\begin{array}{c}\text { Std. Error of } \\
\text { the Estimate }\end{array}$ \\
\hline 1 & $.202^{\mathrm{a}}$ & .041 & .014 & 0.54 \\
2 & $.365^{\mathrm{b}}$ & .133 & .104 & 0.52 \\
3 & $.476^{\mathrm{c}}$ & .226 & .195 & 0.49 \\
4 & $.747^{\mathrm{d}}$ & .559 & .538 & 0.37 \\
\hline
\end{tabular}

a Predictors: Constant, Education, Marital, Tenure, Gender, Age

b Predictors: Constant, Education, Marital, Tenure, Gender, Age, Individual Factors

c Predictors: Constant, Education, Marital, Tenure, Gender, Age, Individual Factors, Situational Factors

d Predictors: Constant, Education, Marital, Tenure, Gender, Age, Individual Factors, Situational Factors, Reaction to Training

Note. Dependent variable: Motivation to Transfer Training

The $R$ square statistics for all four models decreased slightly from the first regression analysis. Moreover, by applying the Stein formula, the $R$ square dropped to .5153 for the factored variables. The beta standardized coefficients, however, were higher than in the first analysis. As presented in Table 14, the individual factor beta in step two was moderately strong and statistically significant $(\beta=.65, p<.01)$ and both 
the individual factor and situational factor were moderately strong and statistically significant in step $3(\beta=.192, p<.01 ; \beta=.340, p<.01)$. When reaction to training was added to the fourth model, individual factors no longer were significant, but the effects for situational factors remained significant $(\beta=.161, \mathrm{p}<.01)$.

\section{Table 14}

Hierarchical Regression Model using Situational and Individual Factors Summary: Motivation to Transfer Training

\begin{tabular}{|c|c|c|c|}
\hline Step and Predictor Variable & $\overline{R^{2}}$ & $\triangle R^{2}$ & $\beta$ \\
\hline Model 1 & .04 & .04 & \\
\hline Age & & & .03 \\
\hline Gender & & & -.07 \\
\hline Marital & & & .00 \\
\hline Tenure & & & $-.21 * *$ \\
\hline Education & & & -.08 \\
\hline Model 2 & .13 & .09 & \\
\hline Age & & & .02 \\
\hline Gender & & & -.04 \\
\hline Marital & & & -.01 \\
\hline Tenure & & & $-1.6^{*}$ \\
\hline Education & & & -.07 \\
\hline Individual Factors & & & $-.31 * *$ \\
\hline Model 3 & .23 & .09 & \\
\hline Age & & & .04 \\
\hline Gender & & & .00 \\
\hline Marital & & & -.02 \\
\hline Tenure & & & -.09 \\
\hline Education & & & -.04 \\
\hline Individual Factors & & & $.19 * *$ \\
\hline Situational Factors & & & $.34 * *$ \\
\hline Model 4 & .56 & .33 & \\
\hline Age & & & .07 \\
\hline Gender & & & .05 \\
\hline Marital & & & -.04 \\
\hline Tenure & & & -.02 \\
\hline Education & & & .07 \\
\hline Individual Factors & & & .06 \\
\hline Situational Factors & & & $.16^{* *}$ \\
\hline Reaction to Training & & & $.65^{* *}$ \\
\hline
\end{tabular}

${ }^{*} p<.05 \quad{ }^{* *} p<.01$ 


\section{Summary}

The purpose of this chapter was to present the results of this study's statistical tests in the form of descriptive statistics and inferential statistics to answer the four research questions. The survey responses in this study reflected a 94\% response rate. Reliability coefficients for the scales used in the study were all above the minimum acceptable level and validated by the source material researchers prior to the study.

Research question one examined the relationship between individual characteristic factors (locus of control, self efficacy, learning confidence, motivation to learn) and motivation to transfer training in a call-center environment. The Pearson correlation coefficient for three of the individual characteristic variables and the criterion variable, motivation to transfer, was moderate and statistically significant $(p=.01)$. Locus of control was the only individual characteristic variable which was not significant at the .01 alpha level.

Research question two examined the relationship between situational characteristic factors (manager support, job support, and organizational support) and motivation to transfer training in a call-center environment. The Pearson correlation coefficient for all three of the individual characteristic variables and the criterion variable was moderate to moderately strong and statistically significant $(p=.01)$. The situational factors as a group were more closely correlated with motivation to transfer than the individual factors. 
Research question three examined the relationship between post-training reaction and motivation to transfer training in a call center environment. The Pearson correlation coefficient for reaction to training and the criterion variable was very strong and statistically significant $(p=.01)$.

Research question four examined the predictive relationship between individual and situational variables and motivation to transfer in a call center environment after controlling for demographic variables. The results from the hierarchical regression analysis showed that individual factors and situational factors accounted for a moderate amount of variance in the criterion variable when entered into the equation. Reaction to training accounted for a large variance in the criterion variable when it was added to the equation. When the individual and situational variable sets were reduced through factor analysis and re-entered into the regression model, the situational factors retained significance in the final model while the individual factors did not $(p=.01)$. The reaction to training variable remained a strong predictor for motivation to transfer variance.

The study was significant in that, with the exception of the first hypothesis concerning locus of control, support was found for all four research questions. Most notably, the moderate to strong predictive relationships between individual, situational, and reaction variables and motivation to transfer indicate participants who possessed the attitudes measured by the predictors also thought they would experience higher transfer to the job. A more detailed discussion of these findings and their implication to practical application and further research is discussed in the next chapter. 


\section{CHAPTER V \\ DISCUSSIONS, IMPLICATIONS, RECOMMENDATIONS AND SUMMARY $\underline{\text { Introduction }}$}

This chapter discusses and summarizes the purpose, results, implications, and recommendations of the current study. The study was guided by four questions which examined various independent variable effects on motivation to transfer training. The first question investigated the relationship between individual characteristic factors and motivation to transfer in a call center environment. The second question examined the relationship between situational characteristic factors and motivation to transfer. The third question examined the relationship between post-training reaction and motivation to transfer. Research question four investigated the predictive relationship between all the aforementioned predictor variables and motivation to transfer.

Chapter five is structured to analyze and interpret the quantitative findings of the study and discuss implications and recommendations for future research and application. The results of this study support prior research in the transfer literature and provide important implications for managers, trainers, human resource professionals, and researchers in the field of performance improvement. Because the contextual setting of 
the study was within a call center environment, the results should also be of interest to call center managers who wish to improve team performance.

Within this chapter, the following sections will be summarized: statement of the problem, review of the theoretical model and method, summary of results, discussion of the results for each of four research questions, limitations, significance, suggestions for additional research, and summary.

\section{$\underline{\text { Statement of the Problem }}$}

This study addressed the trend that both public and private organizations continue to increase their overall spending on training, but without a parallel increase in training transfer to the job. Studies in the literature estimate that only $10-15 \%$ of skills and knowledge acquired during training are actually used on the job (Curry, Caplan, \& Knuppel, 1994; Facteau, Dobbins, Russell, Ladd, \& Kudisch, 1995; Georgenson, 1982). While many studies have measured the factors that influence training transfer, the fact that little transfer research has been conducted within the context of a customer service call center environment was also addressed. It was also noted that there are relatively few studies in the literature focused on individual versus situational factors and how those factors influence a training participant's motivation to transfer. This study investigated the relationships between individual, situational, and reaction to training factors and motivation to transfer training to the job in a customer service call center environment. 


\section{$\underline{\text { Review of the Theoretical Model and Method }}$}

Mathieu and Martineau’s (1997) conceptual model of training motivation was

presented as the foundation of this study. The model asserts that individual and situational characteristics are antecedents to motivation to transfer training to the job. Based on the literature search and this model, several survey questionnaires were chosen and utilized to test the model within the context of a call-center environment. This study used a sample population from a large mid-western insurance company. The participants in the study worked in a customer service call center environment. The independent variables of interest were individual characteristics (locus of control, general selfefficacy, learning confidence, and motivation to learn), situational characteristics (organizational support, managerial support, and job support), and reaction to training. The dependent, or criterion variable was motivation to transfer training. There were also other demographic variables included in the study such as age, ethnicity, marital status, tenure with the company, and educational level. The two main statistical procedures were Pearson’s correlation and hierarchical multiple regression analysis. Additionally, factor analysis was used to reduce the number of individual and situational variables and reduce multicollinearity. Trainee participants completed a questionnaire at the beginning of training comprised of six sub-scales measuring all but one (reaction to training) of the predictor variables. Trainees then completed a second questionnaire at the conclusion of training comprised of a single scale measuring the reaction to training and the criterion variable motivation to transfer training. Data from the returned questionnaires were entered into SPSS and analyzed. 


\section{$\underline{\text { Summary of the Results }}$}

Of the 208 employees attending training who were invited to participate in the survey, 206 chose to participate. Of the 206 pre-training surveys collected, 195 or $95 \%$ exactly matched a corresponding second survey, yielding an effective response rate of 94\% of all employees surveyed. Pearson correlation was used to test the theoretical model and determine the relationships between the predictor variables and the criterion variable. The first three questions were supported by these tests. As predicted by the model and as supported by the literature, positive correlations were found for all predictor variables when compared separately with motivation to transfer training, with the exception of locus of control. The findings on locus of control in this study are consistent with prior research by Cheng (2000) and Noe (1986) who found no significant relationship with motivation to transfer. Reaction to training was most highly correlated with motivation to transfer, followed by the situational characteristic factors, followed by the individual characteristic variables.

For the fourth research question, hierarchical multiple regression was used to test the theoretical model and determine whether the predictor variables had significant relationship with the criterion variable. Four steps were used in the model with demographic variables loading first, followed by individual characteristic variables, situational characteristic variables, and finally the reaction to training variable. Results for this test revealed that the effect of motivation to learn on motivation to transfer was significant through all three models when all variables were considered separately. Reaction to training was by far the strongest predictor for transfer $(\beta=.65, p<.01)$ 
accounting for $26 \%$ of the variance. Due to the high degree of correlation among the variables, however, there was a concern about the effect of multicollinearity. When individual characteristic and situational characteristic variables were reduced to one factor each through factor analysis, and then loaded into the hierarchical regression test, significance for both variables was found in models 2 and 3 . In the final factored model with all variables included, significance was found in both the situational factors variable ( $\beta=.16, p<.01)$ accounting for $9 \%$ of the variance, and reaction to training variable $(\beta=$ $.65, p<.01)$ accounting for $33 \%$ of the variance. These findings support the notion that the level of managerial, organizational, and job support is a key factor in eliciting transfer motivation. The practical significance of these results is discussed in this chapter.

\section{$\underline{\text { Measures Used }}$}

The scales measuring individual and situational characteristics were derived from existing instruments that had been validated by previous research. Locus of control was measured using an 11-item scale originally developed by Andrisani and Nestel (1976) and adapted by Noe (1986). Learning confidence was measured using a five item scale developed by Warr, Allan, and Birdi (1999). General Self-Efficacy was measured using a 17-item scale developed by Sherer, Maddux, Mercadente, Prentice-Dunn, and Rogers (1982). Motivation to learn was measured using a six item scale developed by Noe (1987). Managerial support was measured using a five item scale developed by Tracey, Tannenbaum, and Kavanagh (1995). Job support was measured using a five item scale

developed by Tracey, Tannenbaum, and Kavanagh (1995). Organization support was 
measured using a five item scale developed by Tracey, Tannenbaum, and Kavanagh (1995). Trainee reaction to training was measured using a nine item scale with three subscales developed by Warr, Allan, and Birdi (1999). The criterion variable, motivation to transfer skills to the job was measured using a six-item scale developed by Noe, Schmitt, and Wilk (1993). With the exception of locus of control (.55), the coefficient alphas for all scales as measured by this study were above .70.

\section{$\underline{\text { Research Questions One, Two, and Three: Findings and Implications }}$}

The first research question determined the relationship between predictor variables and motivation to transfer. The Pearson correlations for individual factors, with the exception of locus of control, were moderate. The highest correlation with motivation to transfer was motivation to learn $(r=.36, p<.01)$. These findings are not surprising and support the literature. It suggests that the attitudes and self-concept a learner brings into the training environment play a significant role in how motivated the participant is to transfer training to the job.

Individual Characteristic Variables. General self-efficacy $(r=.18, p<.01)$ was found to be significantly correlated with transfer. Other studies have also found that general selfefficacy is a predictor for transfer (Mathieu, et al, 1992; Martocchio \& Webster, 1992). Motivation to learn $(r=.36, p<.01)$ and that learning confidence $(r=.26, p<.01)$ had an even stronger correlation with transfer. These also support previous research by Warr 
(1999) that found learning confidence significantly predicted change in behavior on the job.

These findings have significant implications for organizations and how they select and prepare employees for training. First, by selecting employees that bring with them the individual characteristics that are highly correlated with training transfer on the job, they would effectively create a workforce that is predisposed to return more of the organization’s training investment. Most organizations have minimal screening processes beyond work history and references. Those that do profile for successful job attributes typically look for competencies that have been proven to correlate with individual productivity. There are few, if any, validated selection systems that correlate a job applicant’s individual characteristics with their ability to learn and transfer learning on the job.

This study's results also build a compelling case for fostering a work environment where discovery, self-learning, and formal learning are enmeshed in the culture of the organization. Bandura (1986) posited that self-efficacy could be enhanced through the use of four sources of information: vicarious experience, verbal persuasion, psychological and affective states, and enactive mastery experience. Of the four, enactive mastery experience, defined as repeated performance accomplishments, has been shown to provide the largest increase in self-efficacy (Gist, 1987). By creating an environment where employees build confidence in their abilities to accomplish learning, self-efficacy is enhanced, learning confidence is increased and the motivation to learn is reinforced. Based on the literature and the results of this study, these attributes will create a higher level of motivation to transfer training back to the job. The learning organization as a 
management trend reached it's apex of popularity nearly 10 years ago and has since been supplanted by other more fashionable practices. Yet transfer research continues to show that motivation to transfer training on the job is related to creating a successful learning environment where employees develop their confidence in their ability to learn and do the things they learn.

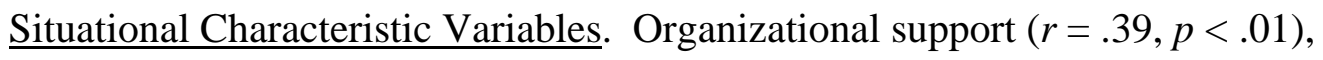
managerial support $(r=.40, p<.01)$, and job support $(r=.40, p<.01)$ exhibited moderately strong correlation to motivation to transfer. These findings reflect over 25 years of similar research supporting managerial and organizational support as an effective catalyst for training transfer. In some of the earliest research on situational transfer variables, Huczynski and Lewis (1980), found that transfer probability was more than double with employees who discussed their training with their supervisors versus those who did not. Brinkerhoff and Montesino (1995) performed an experimental study which showed that training participants who had high training transfer scores were much more likely to have had pre-training discussions with their managers.

The findings in this study, like past research, have major implications in the workplace. Namely, if organizations would invest more time and resources in preparing the organizational climate, managers, and job design to support learners before and after the training, the payoff would far exceed the investment. Of particular interest in this study's data is the strong collinearity exhibited by all three situational variables. This suggests that in the mind of the participant, the job, the manager, and the organization are all perceived as one entity when thinking about support of training transfer. The systemic 
nature of the situational influences around the learner may obviate the need to examine these factors individually. The good news may be that improving support in any one of them may improve the perception of support in all of them and result in higher transfer.

Of course, the correlations discussed in the first three research questions isolate the relationship to transfer without considering moderating or mediating variables, and therefore are more directional than representative of the way things really work. Understanding how the independent variables act on each other and the dependent variable is a more interesting question and will be covered in the next section.

Reaction to Training Variable. The reaction to training variable was strongly correlated with motivation to transfer training $(\mathrm{r}=.72, \mathrm{p}<.01)$. As mentioned in the limitations section of this study, this could be due to the construct of the study itself. Reaction to training was the only variable measured simultaneously with the criterion variable. By answering the intermixed scale questions for both variables in the same setting, the participants may have carried over short term memory impressions of some questions into others. There may also have been the condition Podsakoff (2003) calls consistency motif wherein respondents who have a desire to appear consistent and rational in their responses might search for similarities in certain questions and ensure their responses are mutually supportive. For example, in the current study's post training survey, a participant who reacts favorably to the training program might also feel compelled to affirm that they will use the training on the job.

Setting aside the method bias concerns, however, there is still reason to consider the strong correlation between reaction and motivation to transfer and it's implications 
for training professionals. Prior studies have shown that reaction to training does predict transfer and that there is good reason to understand how participants feel about the training before they return to the job (Alliger, Tannenbaum, Bennett, Traver, and Shotland, 1997).

\section{Research Question Four: Findings and Implications}

In the final model as presented in Figure 2 below, the results of this study supported two of the assertions by Mathieu and Montesino (1994). Situational characteristics, taken as a group, and reaction to training had significant predictive ability for post-training motivation to transfer. With the exception of learning confidence, individual characteristics taken either as a group or separately into the regression model did not have significant effect on the dependent variable, transfer of training.

Training Program Attributes

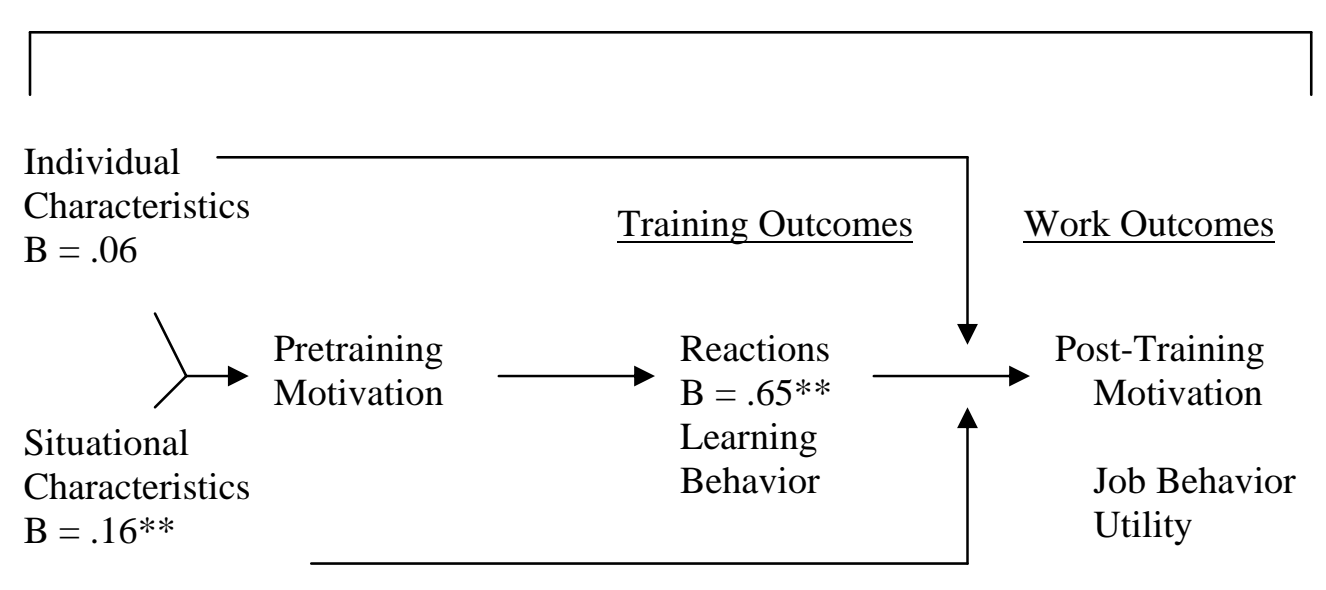

Figure 2: Conceptual Model of Training Motivation (Mathieu \& Martineau, 1997) $* * \mathrm{p}<.01$ 
There are several implications to these results. First, the rather strong effect of the reaction to training variable as it was entered into the model greatly exceeded the influence of almost all variables with the exception of motivation to learn. While we may question the results due to the proximity between the reaction variable and the dependent variable in the data collection method, we cannot discount the predictive strength of this variable. Training professionals would do well to more closely examine reaction to training and compare it to actual job transfer.

Second, the motivation to learn variable remained a significant predictor through all four models of the first regression. Unlike the other three individual predictors, this attribute may provide predictive correlation to training transfer for employee selection systems. The weak correlation of the other three variables, either taken separately or as a group, suggest that apart from motivation to learn, other self-reflective attitudes probably do not have as much influence as situational factors. This has particular significance given that the transfer literature reports individual characteristics like self-efficacy and motivation to learn on equal footing with supervisory and organizational influence. The context and setting of this research in a call center may explain some of the discrepancy and strongly suggests further research. Much of the prior training transfer research was conducted in either academic settings or using soft-skill development training in a business environment. The training in this study, by contrast, was systems-related and very job specific within a highly supervised call center environment. The study suggests that different training transfer strategies may be necessary for different types of organizations. In a less structured organizational culture, individual characteristics may 
be most important to training transfer. In a more highly structured environment, such as in this study, situational characteristics may hold more weight.

A third implication was found in the way the variables entered the model. In the final regression, organizational, managerial, and job support variables, when taken together as one variable, explained a significant amount of motivation to transfer where they failed to do so when examined individually in the prior regression test. This prompts the question of whether employees see any difference between their manager, their organization, and their job design when it comes to motivation to transfer training. Given these findings managers and training professionals should consider the holistic and systemic environment within which an employee learns and not focus on a single situational attribute.

\section{$\underline{\text { Recommendations }}$}

Recommendation One: Develop and implement a selection instrument that screens for motivation to learn attributes that predict for training transfer. Most employee selection instruments focus on integrity or job competency related factors. While helpful in narrowing the applicant population to those who may fit the position, they do little to bring those in who are naturally more inclined to learn and change their behaviors after they are on the job. The results of this study and others show that the individual characteristic motivation to learn plays a significant role in determining how likely a training participant is utilize learned skills in the work environment. 
Recommendation Two: Organizational leaders should foster an environment that increases self-efficacy, learning confidence and motivation to learn and then measure it's effectiveness in terms of training transfer. Create a learning culture that focuses on learning as a means to an end and not just an end. Many companies have accomplished this through implementing personal development curricula, self-paced employee development modules, employee resource and learning centers, and tuition reimbursement programs. Others have adopted an organizational reward system tied to goal setting and learning that created improved training transfer (Wexley \& Baldwin, 1986). While many organizations attempt to create learning cultures, few actually measure the end result of training transfer. This is primarily due to the fact that developing learning organizations takes time and leaders often change, lose focus, or do not have the patience to measure results beyond quarterly time periods. The end result is a cost on the balance sheet that has no justifiable legs to support it. For those who do take a strategic, long term view of learning, however, the research says there should be a pay-off.

Recommendation Three: Implement pre and post training interventions beyond the training program itself. Instructional designers must consider how training transfer interventions can be built into both the pre and post training events. Moreover, these interventions should be tied to the goals and objectives of the training rather than served up as generic discussions. For example, a training module on coaching skills would best be supported by a pre training discussion between participant and manager about opportunities that exist to improve the employee's coaching skills. A post training discussion would review what was learned and establish an action plan and performance 
contract to implement new skills on the job. The manager should then give appropriate feedback on how the employee is progressing in using the new learned skill.

Once the instructional design incorporates both pre and post training events, the participant's managers should be trained on how to coach for training transfer. At the organization level, this manager directed "coaching for transfer training” must not be a one-time event, but rather, initiated periodically with certification. Most organizations make the mistake of believing that training begins and ends with singular training events that are unconnected with the larger system of work. Organizational leaders who want to return more than $10-15 \%$ on their training investment need to consider transfer of training as a systems process. This process should create both context and closure for employees to commit to using their newly learned skills and knowledge in the workplace.

\section{$\underline{\text { Study Limitations }}$}

This study contained possible method variance, training context, and generalizability limitations. Method variance is defined as the variance that is attributable to the method of measurement rather than the theoretical construct of interest (Podsakoff, P. M., MacKenzie, S. B., Lee, J., \& Podsakoff, N. P., 2003). Within this study there are three areas where common method variance may create unwanted covariance between the predictor variables and the criterion variables. 
First the criterion variable, motivation to transfer, is by definition a nonproductive outcome that does not represent observable transfer of training to the job. While studies have shown there is a link between participant motivation and application to the job, the focus of this study is only on the motivation to transfer factor. Using perceptions rather than actual behavior can be problematic in social research because respondents often have a tendency to maintain consistency in their responses when it would not exist in behavior or they create implicit theories that do not represent the true relationship of the variables (Podsakoff, et.al). Whether or not the behavior of training transfer is actually predicted by the independent variables of interest is not addressed in this study. This study specifically addresses the intention or motivation to transfer training immediately after the completion of training.

Another potential method variance limitation was the fact that the respondents provided the measurement for both the predictor variable and criterion variable. This self-report bias may manifest itself in several ways, but the one most likely in this study is the condition of social desirability. Social desirability is the tendency for respondents to place themselves in a favorable way regardless of their actual thoughts and feelings about a subject (Podsakoff et.al, 2003). Participants in this study may have felt that by answering in a particular way to certain questions, they may be viewed more favorably by the organization. The study design attempted to mitigate this bias by ensuring that survey administration was conducted by a trained professional not connected with the employees' line management and ensuring absolute anonymity procedures were used. These procedures will be discussed in more detail in chapter 3 of this study. 
Another possible method variance limitation is the construct and delivery of the questionnaire itself. While self-reports are a convenient and efficient way to measure attitudes, they are also a fallible source of data (Schwarz, 1999). Survey researchers have conducted numerous studies showing that preceding questions may influence a respondent’s answers on subsequent questions (Schuman \& Presser, 1981; Schwarz \& Strack, 1991; Tourangeau \& Rasinski, 1988). Other studies have shown that because a respondent can go back and forth between questions, subsequent questions may also influence preceding ones (Schwarz \& Hippler, 1999). While all respondents answer questions administered in the same order, the collected overall attitude measurement may be influenced by the order of questions. In this sense, the survey used in this study, and in fact any attitude survey becomes a source of information that respondents draw on to form their answers (Schwarz, 1999). We rely on the participant's perspective of their personal motivation at the time of they complete the survey. This motivation could be influenced by the survey itself or could change between the time they complete the survey and the time they have opportunity to use their newly learned skills and knowledge on the job. The time and location of the survey used in this study may also be a source of covariance between the reaction to training predictor variable, and the criterion variable, motivation to transfer. Because these variables were measured from the same survey questionnaire at the same time, it is possible that answers to both types of questions may co-exist in short term memory and cause correlation in responses. One remedy for this condition is to separate the predictor variable measurement from the criterion variable measurement by space and time (Podsakoff et.al, 2003). While this was affected for all other predictor variables, the reaction to training variable, by logical 
necessity could only be measured at the same time as the motivation to transfer variable, and therefore remains a limitation of the study.

Finally, the research was conducted in the context of technical skills courses. This may have influenced the respondent's motivation to transfer regardless of their responses to the predictor variables because of the organization's expectation that these skills must be used on the job. Had the study focused on more discretionary areas such as communication style, management and supervisory skills, or socialization skills, the importance of the predictor variables may have been different. Additionally, the industry context is narrow by design and therefore may reduce the generazalibility of the findings to other contexts and settings. Very little research on training transfer within a call center appears in the body of literature and this research is targeted specifically at that audience. While the findings may be directional and somewhat generalizable to other settings, there is no assertion that they will be generalizable.

\section{Suggestions for Additional Research}

The results of this study suggest there are other areas of research that need to be explored with regard to situational and individual factors and training transfer. The following are four such areas.

First, more inquiry needs to be made on how employees delineate between managerial, organization, and job support as situational antecedents to transfer. The high correlations between these variables in this research suggest that employees see them as somewhat interchangeable. The question remains of whether that perception is 
applicable to both a highly structured environment (as in this study) and more autonomous cultures. A study could be done comparing the motivation to transfer measures for employees in both a highly structured organization vs. a highly autonomous organization to understand whether perceptions of situational support leading to transfer change with the nature of the job.

Second, following the recommendation by Mathieu and Martineau (1997) further research on situational constraints need to be investigated on several levels. There may be many types of situational constraints that cannot be summarized in the scope of the scale used in this study. For example, if there are staffing level deficiencies, or time pressures, or poor management or policy practices, motivation to transfer might be compromised even if the perception of overall support is positive. Further understanding of job inhibitors is essential to understand all the variables affecting an employee's willingness and ability to exhibit transfer behaviors. A longitudinal study could be conducted

Third, the strong relationship between reaction to training and training transfer needs to be examined more closely. Training professionals typically eschew "smile sheets” as not indicative of training effectiveness, but as Alliger and others have shown, reaction to training does predict transfer in many cases (Alliger, Tannenbaum, Bennett, Traver, and Shotland, 1997). The relevant question then is how can both reaction and motivation to transfer be measured in the same time period, but separated in such a way so as not cause common method bias? Both scales must be administered after the training for obvious reasons, but given too far after training presents the possibility that an intervening variable may influence the outcome. The author suggests conducting a 
study wherein participants are given a short activity or break to disassociate one survey from the other, but still within the close proximity to the end of training.

Finally, the question of whether motivation equates to actual behavior on the job is perhaps the most salient question that needs much deeper inquiry. How does motivation to transfer correlate with actual job transfer? Capturing a subject's intention is often easier than identifying their actual behaviors. There are at least two plausible ways a study of this nature could be designed. First, two surveys could be administered, one focusing on motivation to transfer, the other asking for perception of behavior that supported transfer. Perception bias would obviously be a factor in such a design. Second, direct observation of the transfer behavior is more accurate, but harder to identify.

\section{Summary}

It was almost fifty years ago when Mosel (1957) said that training often "makes little or no difference in job behavior.” Despite advances in instructional technology and computer technology, this statement is still valid today. Our best training results are still dependent upon the way in which the learner is supported both before and after training on the job. The present study indicates that within the context of a call center environment, situational characteristic variables will predict for motivation to transfer training to the job. Specifically, how one’s manager, job, and organization support the learning events in training have a direct correlation to whether they will be used. Even the best instructional design can be thwarted by poor external support. 
The study indicates that reaction to training is a good predictor for motivation to transfer. While more research is called for in this area, this study gives further support for not tossing the "smile sheets" and better understanding their relevance to transfer. The study results also imply that individual characteristics, and specifically motivation to learn, though not a significant factor in the final regression model, correlates significantly when individually compared to motivation to transfer.

Finally, the study presents these findings in the context of a call center environment. With the growth of the customer service oriented workplace both in the U.S. and internationally, these results help us better understand the relationship of situational characteristics to individual characteristics and how both predict motivation to transfer in a call center environment. 


\section{REFERENCES}

Alliger, G.M., Tannenbaum, S.I., Bennett, Jr., W., Traver, H., \& Shotland, A. (1997). A meta-analysis of the relations among training criteria. Personnel Psychology, 50, 341-358.

Andrisani, P. J., \& Nestel, G. (1976). Internal-external control as contributor to and outcome of work experience. Journal of Applied Psychology, 61, 156-165.

Baldwin, T. T., \& Ford, K. J. (1988). Transfer of Training: A Review and Directions for Future Research. Personnel Psychology, 41, 63-105.

Bandura, A. (1978). Reflections on self-efficacy. Advances in Behavioral Research and Therapy, 1, 237-269.

Bates, R. A., Holton, E. F. III, \& Seyler, D. L. (2000). The role of interpersonal factors in the application of computer-based training in an industrial setting. Human Resource Development International, 3, 19-42.

Becker, T. E., \& Klimoski, R. J. (1989). A field study of the relationship between the organizational feedback environment and performance. Personnel Psychology, 42(2), 343-358

Brinkerhoff, R. O., \& Montesino, M. U. (1995). Partnerships for training transfer: Lessons from a corporate study. Human Resource Development Quarterly, 6(3), 263-274.

Broad, M. L., \& Newstrom, J. W. (1992). Transfer of training: Action-packed strategies to ensure high payoff from training investments (1s1 ed.). Reading, Massachusetts: Addison-Wesley Publishing Company.

Broad, M. L. (2005). Beyond transfer of training. San Francisco: Pfeiffer

Brown, S. P., \& Leigh, T. W., (1996). A new look at psychological climate and its relationship to job involvement, effort, and performance. Journal of Applied Psychology, 81(4), 358-368.

Burke, L. A., \& Baldwin, T. T. (1996). Improving transfer of training: A field investigation of the effects of relapse prevention training and transfer climate on maintenance outcomes. Paper presented at the Academy of Management Proceedings. 
Byham, C., Adams, D., \& Kiggins, A. (1976). Transfer of modeling training to the job. Personnel Psychology, 29(3), 345-349.

Cammann, C., Fichman, M., Jenkins, D., \& Klesh, J. (1979). The Michigan Organizational Assessment Questionnaire. Unpublished manuscript, University of Michigan.

Cheng, E. W. L. (2000). Test of the MBA knowledge and skills transfer. International Journal of Human Resource Management, 11(4), 837-852.

Clarke, N. (2002). Job/Work Environment Factors Influencing Training Transfer within a Human Service Agency: Some Indicative Support for Baldwin and Ford's Transfer Climate Construct. International Journal of Training and Development, 6(3), 146-162.

Compeau, D., Higgins, C. A., \& Huff, S. (1999). Social cognitive theory and individual reactions to computing technology: a longitudinal study. MIS Quarterly, 23(2), 145-158.

Curry, D., Caplan, P., \& Knuppel, J. (1994). Transfer of training and adult learning. Journal of Continuing Social Work Education. 6(1), 8-14.

Delaney, J. T. , \& Huselid M. A. (1996). The impact of human resource management practices on perceptions of organizational performance. Academy of Management Journal, 39(4), 949-969.

The Economist, Special Report: Democracy's drawbacks - Reform in India, London: Oct 29, 2005. Vol. 377(8450), p. 23

Egan, T. M., Yang, B., \& Bartlett, K. R. (2004). The effects of organizational learning culture and job satisfaction on motivation to transfer learning and turnover intention. Human Resource Development Quarterly, 15(3), 279-301.

Facteau, J. D., Dobbins, G. H., Russell, J. E. A., Ladd, R. T., \& Kudisch, J. D. (1995). The Influence of General Perceptions of Training Environment on Pretraining Motivation and Perceived Training Transfer. Journal of Management, 21(1), 125.

Ford, J. K., Quinones, M. A., Sego, D. J., \& Sorra, J. S. (1992). Factors affecting the opportunity to perform trained tasks on the job. Personnel Psychology, 45, 511527.

Foxon, M. (1993) A process approach to the transfer of training. Australian Journal of Educational Technology, 9(2), 130-143. 
Gans, N., Koole, G., Mandelbaum, A. (2003). Telephone Call Centers: Tutorial, Review, and Research Prospects. Manufacturing \& Service Operations Management, 5(2), 79-141.

Georgenson, D., (1982). The problem of transfer calls for partnership. Training and Development Journal, 36(10), 75-78.

Gist, M. E. (1987). Self-efficacy: Implications for organizational behavior and human resource management. Academy of Management Review, 12, 472-485.

Gist, M. E., Stevens, C. K., Bavetta, A. G. (1991). Effects of self-efficacy and posttraining intervention on the acquisition and maintenance of complex interpersonal skills.

Gist, M. E., Bavetta, A. G., \& Stevens, C. K. (1990b). Transfer training method: Its influence on skill generalization, skill repetition, and performance level. Personnel Psychology, 43(3), 501-523.

Gist, M. E., Bavetta, A. G., \& Stevens, C. K. (1990a). The effectiveness of self management versus goal setting in facilitating training transfer. Proceedings of the 50th Annual Meeting of the Academy of Management, 117-121.

Goldstein, I. L. (1993). Training in organizations, Belmont, CA: Brooks/Cole.

Green, D. M., (2003). Self-Efficacy: A communication model for the development of self-efficacy in the classroom. Journal of Teaching in Social Work, 23, (3/4) 107116.

Haccoun, R. T., \& Saks, A. M. (1998). Training in the 21st century: some lessons from the last one. Canadian Psychology, 39(1-2), 33-51.

Holton III, E. F., \& Bates, R. A. (2000). Development of a Generalized Learning Transfer System Inventory. Human Resource Development Quarterly, 11(4), 333-351.

Holton III, E. F., Ruona, W. E. A., \& Leimbach, M. (1998). Development and validation of a generalized learning transfer climate questionnaire: Final research. Academy of Human Resource Development 1998 conference proceedings, 482-489.

Holton, E. F., III, Chen, H.-C., \& Naquin, S. S. (2003). An examination of learning transfer systems across organizational settings. Human Resource Development Quarterly, 14, 459-482.

Huczynski, A. A., \& Lewis, J. W. (1980). An empirical study into the learning transfer process in management training. The Journal of Management Studies, 17, 227240 . 
Irving, G., Coleman, D. F., \& Cooper, C. L. (1997). Further assessment of a threecomponent model of occupational commitment: generalizability and differences across occupations. Journal of Applied Psychology, 82, 444-452.

Kahn, W. A. (1990). Psychological conditions of personal engagement and disengagement at work. Academy of Management Journal, 33, 692-724.

Kearney, K. A. (1984). Self-Generated Identification Codes for Anonymous Collection of Longitudinal Questionnaire Data. Public Opinion Quarterly, 48, 370-378.

Machin, M. A., \& Fogarty, G. J. (2004). Assessing the antecedents of transfer intentions in a training context. International Journal of Training and Development, 8(3), 222-236.

Martocchio, J. J., \& Webster, J. (1992). Effects of feedback and cognitive playfulness on performance in microcomputer software training. Personnel Psychology, 45, 553578.

Marx, R. D. (1986). Improving management development through relapse prevention strategies. Journal of Management Development, 5(2), 27-40.

Mathieu, J. E., \& Martineau, J. W., (1997). Individual and situational influences on training motivation. (In Ford, J.K., S.W.J. Kozlowski, K. Kraiger, E. Salas \& M.S. Teachout (Eds.), Improving training effectiveness in work organizations. (pp. 193-221). Mahwah, NJ: Lawrence, NJ: Lawrence Erlbaum Associates.

Mathieu, J. E., Tannenbaum, S. I., \& Salas, E. (1992). Influences of individual and situational characteristics on measures of training effectiveness. Academy of Management Journal, 35(4), 828-846.

May, G. L., \& Kahnweiler, W. M. (2000). The Effect of a Mastery Practice Design on Learning and Transfer in Behavior Modeling Training. Personnel Psychology, 53(2), 353.

Montesino, M. U. (2002). Strategic Alignment of Training, Transfer-Enhancing Behaviors, and Training Usage: A Post-training Study. Human Resource Development Quarterly, 13(1), 89-108.

Mosel, J. (1957). Why training programs fail to carry over. Personnel, 56-64.

Noe, R. A., \& Schmitt, N. (1986). The influence of trainee attitudes on training effectiveness: Test of a model. Personnel Psychology, 39, 497-523.

Noe, R. A. (1986). Trainees' attributes and attitudes: Neglected influences on training effectiveness. Academy of Management Review, 11(4), 736-749 
Nunally J. \& Bernstein, I. H. (1994). Psychometric theory. New York: McGraw-Hill.

Orpen, C. (1999). The influence of the training environment on trainee motivation and perceived training quality. International Journal of Training Development, 3(1), 34-43.

Pate, J., Graeme, M., Beaumont, P., \& McGoldrick, J. (2000). Company-based lifelong learning: what's the pay-off for employers. Journal of European Industrial Training, 24, 149-157.

Podsakoff, P. M., MacKenzie, S. B., Lee, J., \& Podsakoff, N. P. (2003). Common method biases in behavioral research: A critical review of the literature and recommended remedies. Journal of Applied Psychology, 88, 879-903.

Richman-Hirsch, W. L. (2001). Post-training Interventions to Enhance Transfer: The Moderating Effects of Work Environments. Human Resource Development Quarterly, 12(2), 105-120.

Rouiller, J. Z., \& Goldstein, I., L. (1993). The relationship between organizational transfer climate and positive transfer of training. Human Resource Development Quarterly, 4(4), 377-390.

Rouiller, J. Z. (1989). Determinants of the climate for transfer of training. (Doctoral dissertation, University of Maryland, 1989). Dissertation Abstracts International, 50, 07B

Ruona, W. E. A., Leimbach, M., Holton, E. F. III, \& Bates, R. A. (2002). The relationship between learner utility reactions and predictors of learning transfer among trainees. International Journal of Training and Development, 6, 218-228.

Ryman, D. H., \& Biersner, R. J., (1975). Attitudes predictive of diving training success. Personnel Psychology, 28(2), 121-188

Schuman, H., \& Presser, S. (1981). Questions and answers in attitude surveys. New York: Academic Press.

Schwarz, N. (1999). Self-reports: How the questions shape the answers. American Psychologist, 54(2), 93-105.

Schwarz, N., \& Strack, F. (1991). Context effects in attitude surveys: Applying cognitive theory to social research. European Review of Social Psychology, 2, 31-50.

Seyler, D. L., Holton III, E. F., Bates, R. A., Burnett, M. F., \& Carvallho, M. A. (1998). Factors affecting motivation to transfer training. International Journal of Training Development, 2(1), 2-16. 
Sherer, M., Maddux, J., Mercandante, B., Prentice-Dunn, B., Jacobs, B., \& Rogers, R. (1982). The self-efficacy Scale: Construct and Validation. Psychological Reports, $51,663-671$.

Swinney, J. M. (1989). Who's gonna turn the crank OR Implementing training or performance improvement projects. Performance \& Instruction, 28(1), 33-37.

Tannenbaum, S. I., \& Mathieu, J. E. (1991). Meeting trainees' expectations: The influence of training fulfillment on the development of commitment, self-efficacy, and motivation. Journal of Applied Psychology, 76(6), 759-769.

Tannenbaum, S. I. \& Yukl, G. (1992). Training and development in work organizations. Annual Review of Psychology, 43, 399-441.

Taylor, M. C. (2000). Transfer of learning in workplace literacy programs. Adult Basic Education, 10(1), 3-20.

Tourangeau, R. (1992). Attitudes as memory structures: Belief sampling and context effects. In N. Schwarz \& Sudman (Eds.), Context effects in social and psychological research (pp. 35-47). New York: Springer Verlag.

Tracey, J. B., Hinkin, T. R., Tannenbaum, S., \& Mathieu, J. E. (2001). The influence of individual characteristics and the work environment on varying levels of training outcomes. Human Resource Development Quarterly, 12(1), 5-23.

Tracey, J. B., Tannenbaum, S. I., \& Kavanagh, M. J. (1995). Applying trained skills on the job: The importance of the work environment. Journal of Applied Psychology, 80(2), 239-252.

Tziner, A., \& Haccoun, R. T. (1991). Personal and situational characteristics influencing the effectiveness of transfer of training improvement strategies. Journal of Occupational Psychology, 64, 167-177.

van der Klink, M., Gielen, E., \& Nauta, C. (2001). Supervisory Support as a Major Condition to Enhance Transfer. International Journal of Training and Development, 5(1), 52-63.

Vogt, Paul W. (1999). Dictionary of Statistics and Methodology. Thousand Oaks, California: Sage.

Vroom, V.H. (1964). Work and motivation. New York: Wiley

Warr, P, Allan, C., Birdi, K., (1999). Predicting three levels of training outcome. Journal of Occupational and Organizational Psychology , 72, 351-375. 
Warr, P, \& Bunce D. (1995). Trainee characteristics and the outcomes of open learning. Personnel Psychology, 48 (2) 347-375.

Watkins, K. E. a. M., Victoria J. (2003). Make learning count! Diagnosing the learning culture in organizations. Advances in Developing Human Resources, 5(2).

Watson, D., Clark, L. A., \& Tellegen, A. (1988). Development and validation of brief measures of positive and negative affect. Journal of Personality and Social Psychology, 54(6), 1062-1070.

Werner, J. M., O'Leary-Kelly, Baldwin, T. T., \& Wexley, K. N. (1994). Augmenting behavior-modeling training: Testing the effects of pre- and post-training interventions. Human Resource Development Quarterly, 5(2), 169-183.

Wexley, K. N., \& Baldwin, T. T. (1986). Posttraining Strategies for Facilitating Positive Transfer: An Empirical Exploration. Academy of Management Journal, 29(3), 503-520.

Xiao, J. (1996). The relationship between organization factors and the transfer of training in the electronics industry in Shenzhen, China. Human Resource Development Quarterly, 7(1), 55-73. 
Appendix 1

\author{
Human Subject Review \\ University of Louisville
}

\author{
Subject Informed Consent Document \\ Factors Affecting Motivation to Transfer Training
}

\title{
Introduction and Background Information
}

You are invited to participate in a research study. The study is being conducted by Dr. Mike Boyle and Eric T. Hicks (graduate student). The study is sponsored by the University of Louisville, Department of Leadership Foundations and Human Resource Education. The study will take place at ABC Corporation's Louisville training facility. Approximately 200 subjects will be invited to participate.

\section{Purpose}

The purpose of this study is to investigate how people are motivated to use what they learn in training back on the job. The information will be used to help improve training effectiveness at both ABC Corp. and in other organizations.

\section{Procedures}

In this study, you will be asked to complete two surveys, one at the beginning of training, and one at the end of training. These surveys will ask about your learning style, how you think, and your perspectives on management, job and organizational support for training. The first survey will take approximately 15 minutes to complete. The second survey will take approximately 10 minutes to complete. You may decline to answer any questions that make you uncomfortable. In order to maintain anonymity, you will not write your name on either survey, but will write a self-generated identification code which will be used to link the first survey with the second survey.

\section{Potential Risks}

Although there are no foreseeable risks associated with completing this survey, there may be unforeseen risks. 


\section{Benefits}

Although the information collected may not benefit you directly, the possible benefits of this study include improving future training programs. The information collected may not benefit you directly. However, the information learned in this study may be helpful to others.

\section{Confidentiality}

Although total privacy cannot be guaranteed, your privacy will be protected to the extent permitted by law. The study sponsor, the institutional Review Board (IRB), the Human Subjects Protection Program Office (HSPPO), or other appropriate agencies may inspect your research records. Should the data collected in this research study be published, your identity will not be revealed. Data collected in this study will be kept in a password protected computer.

\section{Voluntary Participation}

Taking part in this study is voluntary. You may choose not to take part at all. If you decide to be in this study you may stop taking part at any time. If you decide not to be in this study or if you stop taking part at any time, you will not lose any benefits for which you may qualify.

\section{Research Subject’s Rights, Questions, Concerns, and Complaints}

You acknowledge that all your present questions have been answered in language you can understand and all future questions will be treated in the same manner. If you have any questions about the study, please contact Dr. Mike Boyle at 852-1645 or Eric Hicks at 241-8673. If you have any questions about your rights as a research subject, concerns or complaints about the research staff, you may call the HSPPO (502) 852-5188. You will be given the opportunity to discuss any questions about your rights as a research subject, in confidence with a member of the IRB. The IRB is an independent committee composed of members of the University community, staff of the institutions, as well as lay members of the community not connected with these institutions. The IRB has reviewed this study.

If you want to speak to a person outside the University, you may call 1-877-852-1167. You will be given the chance to talk about any questions, concerns or complaints in 
secret. This is a 24 hour hot line answered by people who do not work at the University of Louisville.

This paper tells you what will happen during the study if you choose to take part. Your signature means that this study has been discussed with you, that your questions have been answered, and that you will take part in the study. This informed consent document is not a contract. You are not giving up any legal rights by signing this informed consent document. You will be given a signed copy of this paper to keep for your records.

Printed Name of Subject

Date Signed

Signature of Subject

Date Signed

Signature of Investigator

Date Signed

Signature of Person Explaining the Consent Form

Date Signed

(if other than the Investigator)

\section{LIST OF INVESTIGATORS}

Dr. Mike Boyle

Eric Hicks
PHONE NUMBERS

(502) 852-1645

(502) 241-8673

Appendix 2 


\section{Self-Generated Identification Number Instructions}

Because you will take this survey in two parts at two different times, we will use a 7character self-generated identification number to link the two surveys together. This is done to preserve your anonymity and avoid the use of personal unique identifiers (such as social security numbers). These codes will be destroyed at the conclusion of the study. Your self-generated ID number will be created by using the following information:

First letter of middle name, first letter of mother's first name, gender ( $\mathrm{M}$ or F), Birth month number, first letter of birth state (or country if not U.S.), number of older brothers and sisters, first number of street address.

For example, if my middle name is Tanya, my mother's name is Carol, I am female, my birth month is July, I was born in Indiana, I have 2 older brothers and 1 older sister (3 total), and my address is 713 Oak Street, then my self-generated identification code would be as follows:

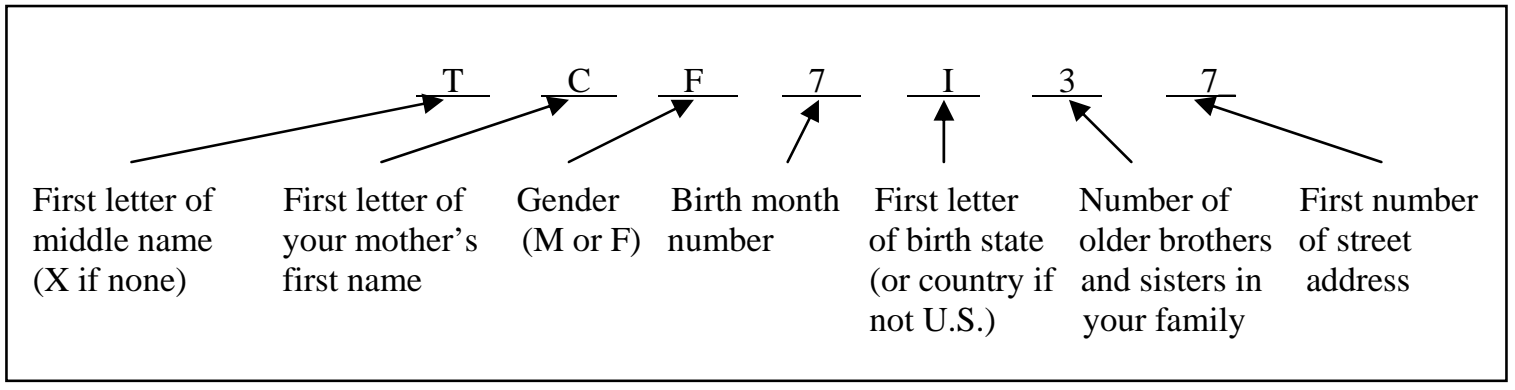

1. Please write your Self-Generated Identification Code as just described in the box below:

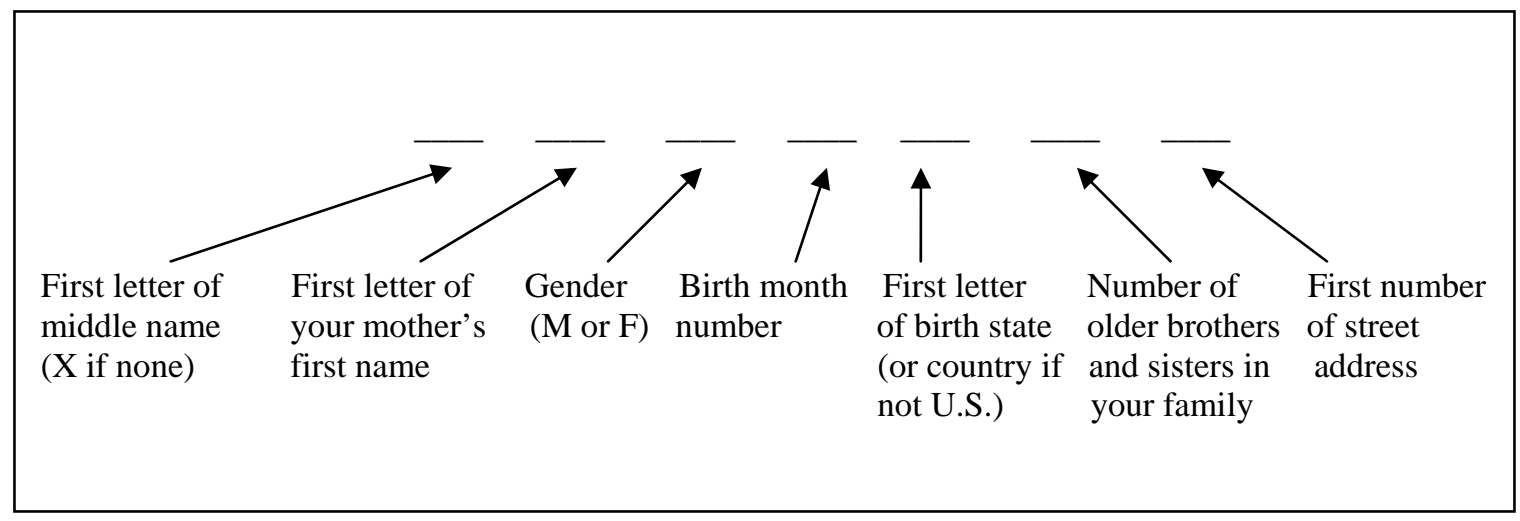

2. Write the Self-Generated ID code you just created on the top of the first page of the survey form in the space provided. 


\section{Appendix 3}

\section{Training Transfer Survey \#1}

Background Information

Date:
ID\#

(7 digit self-generated ID Code)

Age:

Gender: (M or F)

Ethnicity (Check one):

African American

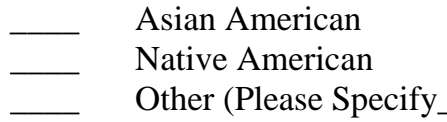

Hispanic American

Marital Status (Check one):

Married Single Tenure with $\mathrm{ABC}$ yrs. mos.

Education Level (Check one): did not graduate from high school High School graduate Two year college graduate Four year college graduate Master's degree Doctoral or professional degree

\section{Circle one number for each question.}

Please answer the following questions as you think about yourself.

1. In the long run, people get the respect they deserve in this world.

2. When I set important goals for myself, I rarely achieve them.

3. When I have something unpleasant to do, I stick with it until I finish it.

4. In the long run, the bad things that happen to us are balanced by the good ones.

5. Generally, I prefer to keep away from training courses.

6. Most people don't realize the extent to which their lives are controlled by accidental happenings.

7. I don't have many problems picking up new skills.

8. Without the right breaks, one cannot be a good leader.

9. I like to learn more about the subjects covered in this course.

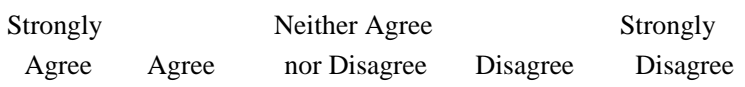

5

4

3

2

1

5

4

3

2

1

5

4

3

2

1

5

4

3

2

1

5

4

3

2

1

5

4

3

2

1

5

4

3

2

1

5

4

3

2

1

5

4

3

2

1 


\section{Circle one number for each question.}

Please answer the following questions as you think about yourself.

10. When I decide to do something, I go right to work on it.

11. I feel tense about this course.

12. I am usually a very good learner.

13. Many times I feel I have little influence over those things that happen to me.

14. I expect that this course will help me a lot in the future.

15. If something looks too complicated, I will not even bother to try it.

16. Who gets promoted often depends on who was lucky enough to be in the right place first.

17. I have no fears about this course.

18. When trying to learn something new, I soon give up if I am not initially successful.

19. I do not seem capable of dealing with most problems that come up in my life.

20. When I make plans, I am certain I can make them work.

21. Generally I am enthusiastic about learning new things.

22. I avoid trying to learn new things when they look too difficult for me.

23. One of my problems is that I cannot get down to work when I should.

24. This course is really a waste of time.

25. When unexpected problems occur, I don't handle them well.

26. I feel insecure about my ability to do things. 5

27. When I make plans, I am almost certain I can make them work.

\begin{tabular}{|c|c|c|c|c|}
\hline Strongly & & Neither Agree & & Strongly \\
\hline Agree & Agree & nor Disagree & Disagree & Disagree \\
\hline 5 & 4 & 3 & 2 & 1 \\
\hline 5 & 4 & 3 & 2 & 1 \\
\hline 5 & 4 & 3 & 2 & 1 \\
\hline 5 & 4 & 3 & 2 & 1 \\
\hline 5 & 4 & 3 & 2 & 1 \\
\hline 5 & 4 & 3 & 2 & 1 \\
\hline 5 & 4 & 3 & 2 & 1 \\
\hline 5 & 4 & 3 & 2 & 1 \\
\hline 5 & 4 & 3 & 2 & 1 \\
\hline 5 & 4 & 3 & 2 & 1 \\
\hline 5 & 4 & 3 & 2 & 1 \\
\hline 5 & 4 & 3 & 2 & 1 \\
\hline 5 & 4 & 3 & 2 & 1 \\
\hline 5 & 4 & 3 & 2 & 1 \\
\hline 5 & 4 & 3 & 2 & 1 \\
\hline 5 & 4 & 3 & 2 & 1 \\
\hline 5 & 4 & 3 & 2 & 1 \\
\hline 5 & 4 & 3 & 2 & 1 \\
\hline
\end{tabular}




\section{Circle one number for each question.}

Please answer the following questions as you think about yourself.

28. If I can't do a job the first time, I keep trying until I can.

29. Becoming a success is a matter of hard work; luck has little or nothing to do with it.

30. I give up easily.

31. In my case, getting what I want has little to do with luck.

32. Many of the unhappy things in people's lives are partly due to bad luck.

33. I am quite nervous about coming to this course.

34. Generally, I like to take up any learning opportunity offered to me.

35. What happens to me is of my own doing.

36. Failure just makes me try harder.

37. I avoid facing difficulties.

38. I am a self-reliant person.

answer the following questions as you think about your organization.

39. This organization rewards employees for using newly acquired knowledge and skills on the job.

40. Independent and innovative thinking are encouraged by supervisors

41. There is a strong belief that continuous learning is important to successful job performance.

42. Supervisors match associates' needs for personal and professional development with opportunities to attend

training.

\begin{tabular}{|c|c|c|c|c|}
\hline $\begin{array}{c}\text { Strongly } \\
\text { Agree }\end{array}$ & Agree & $\begin{array}{l}\text { Neither Agree } \\
\text { nor Disagree }\end{array}$ & Disagree & $\begin{array}{l}\text { Strongly } \\
\text { Disagree }\end{array}$ \\
\hline 5 & 4 & 3 & 2 & 1 \\
\hline 5 & 4 & 3 & 2 & 1 \\
\hline 5 & 4 & 3 & 2 & 1 \\
\hline 5 & 4 & 3 & 2 & 1 \\
\hline 5 & 4 & 3 & 2 & 1 \\
\hline 5 & 4 & 3 & 2 & 1 \\
\hline 5 & 4 & 3 & 2 & 1 \\
\hline 5 & 4 & 3 & 2 & 1 \\
\hline 5 & 4 & 3 & 2 & 1 \\
\hline 5 & 4 & 3 & 2 & 1 \\
\hline 5 & 4 & 3 & 2 & 1 \\
\hline
\end{tabular}

Circle one number for each question.

$\begin{array}{ccccc}\text { Strongly } & & \text { Neither Agree } & & \text { Strongly } \\ \text { Agree } & \text { Agree } & \text { nor Disagree } & \text { Disagree } & \text { Disagree }\end{array}$

5

32

1

5

4

3

2

1

5

4

3

1

5

4

3

2

1 


\section{Circle one number for each question.}

Please answer the following questions as you think about your organization.

43. Learning new ways of performing work is valued in this organization.

44. Job assignments are designed to promote personal development.

45. There are rewards and incentives for acquiring and using new knowledge and skills in one's job.

46. Work assignments include opportunities to learn new techniques and procedures for improving performance.

47. Supervisors give recognition and credit to those who apply new knowledge and skills to their work.

48. Employees are provided with resources necessary to acquire and use new knowledge and skills.

49. Gaining new information about ways to perform work more effectively is important in this organization.

50. There is a performance appraisal system that ties financial rewards to use of newly acquired knowledge and skills.

51. Top management expects high levels of performance at all times.

52. Top management expects continuing technical excellence and competence.

53. This organization offers excellent training programs.

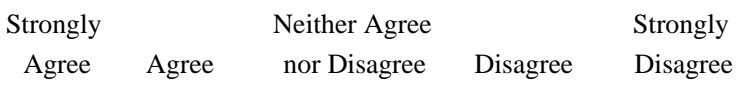

5

4

3

2

1

5

4

3

2

1

5

4

3

2

1

5

4

3

2

1

5

4

3

2

1

5

4

3

2

1

4

3

2

1

5

4

3

2

1

5

4

3

2

1

5

4

3

2

1

5

4

3

2

1

Please return your completed survey to the survey administrator. Thank you for your participation! 


\section{Training Transfer Survey \#2}

Please answer the following questions as you think about yourself.

1. The atmosphere in this course was conducive to learning.

2. My job performance will improve if I use the skills I learned in this course.

3. Communications concerning the activities in this course were clear and adequate.

4. I learned skills in this course that I intend to use in my everyday work.

5. This course was well planned and organized. 5

6. The level of material presented in this course was neither too easy nor too difficult.

7. The skills I learned in this course will be helpful in solving work related problems.

8. There was a good balance between trainer input (lecture) and participant inputs (involvement via discussion and group activity).

9. This course provided me with sufficient opportunities to learn and practice the key behaviors related to the skills I want to improve.

10. I feel capable of using the skills developed in this course in my everyday work.

11. The daily schedule of activities for this course was too demanding.

12. The quality of materials and assignments used in this course were satisfactory.

13. The trainer(s) were easy to understand.

14. I know of work situations in which I plan to use what I have learned in this course.
ID\#

(7 digit self-generated ID Code)

Circle one number for each question.

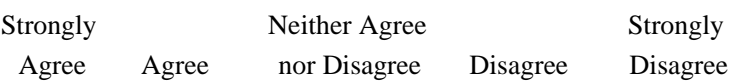

5

4

3

2

1

5

4

3

2

1

5

4

3

2

1

5

4

3

2

1

4

4

3

2

1

5

4

3

1

5

4

3

1

5

4

3

2

1

5

4

3

2

1

5

4

3

2

1

5

4

3

2

1

5

4

3

2

1

5

4

3

2

1

5

4

3

2

1 
15. The trainers helped to create an learning.

16. I believe it is unrealistic to try and use the skills emphasized in this course in my job.

17. I would have liked to have received more

5 5

4 3 feedback regarding my behavior during this course.

Please return your completed survey to the survey administrator. Thank you for your participation! 


\section{CURRICULUM VITAE}

\section{Eric T. Hicks}

110 Sedley Ct.

Pewee Valley, KY 40056

\section{Academic Background}

\section{Graduate}

\section{- Doctor of Philosophy}

Dissertation Title: Individual and Situational Factors affecting 2006

Transfer of Training in a Call Center Environment

Major: Educational Leadership and Organizational Development

UNIVERSITY OF LOUISVILLE, LOUISVILLE, KENTUCKY

- Master of Science

1984

Major: Instructional Systems Technology

INDIANA UNIVERSITY, BLOOMINGTON, INDIANA

\section{Undergraduate}

- Bachelor of Arts

1982

Major: Broadcast Communication

BRIGHAM YOUNG UNIVERSITY, PROVO, UTAH

\section{Professional Experience}

\section{JPMorganChase}

Sr. Director, Performance Improvement

Wilmington, DE. 1997 - Present

Sr. Vice President

First Vice President

Vice President

Strategic and tactical leader for Training, Performance Improvement, Scorecard, and Communications efforts within largest U.S. credit card issuer (87 million Card members, $\$ 125$ billion in outstanding loans). Direct responsibility for team of 227 in improving operational performance and job skills across nine call centers in three countries, ten major functions, and 20,000+ employees. $\$ 15 \mathrm{~mm}$ budget responsibility. 


\section{$\underline{\text { PepsiCo, KFC Division }}$}

Director, USA Training Implementation

Louisville, KY 1992 - 1997

\section{Division Training Manager}

\section{Region Human Resources Manager}

Functional leader for front-line management and skills training within a \$3.7 billion quick service restaurant company. Directly managed department of 30 training professionals and six national training centers. Also led headquarters based training team that designed and implemented new product training for all domestic store operations (1973 units) and franchise operations (3100 units). Department budget of \$10.2 million.

\section{Foremost Corporation of America}

\section{Manager, Corp. Training and Development $\quad$ Grand Rapids, MI 1987 - 1990}

Directed corporate training team of six in designing, developing, and delivering all training activities in a $\$ 1+$ billion property casualty insurance company. Conducted management development and interpersonal skills classes. Led team that designed and developed policy services and claims processing training

\section{NCR Corporation, Dayton, $\mathrm{OH}$}

\section{Project Manager, New Technology Training Instructional Designer, New Technology Training \\ Dayton, OH 1985 - 1987}

Managed project teams in designing and producing interactive media training for 6000 field engineers within $\$ 5.5$ billion computer hardware and software company. Developed and managed multi-media self-paced learning centers. 